The University of Southern Mississippi

The Aquila Digital Community

Master's Theses

Summer 8-1-2015

\title{
Zebrafish and Conditioned Place Preference: A Translational Model of Drug Reward
}

Adam Douglas Collier

University of Southern Mississippi

Follow this and additional works at: https://aquila.usm.edu/masters_theses

Part of the Biological Psychology Commons

\section{Recommended Citation}

Collier, Adam Douglas, "Zebrafish and Conditioned Place Preference: A Translational Model of Drug Reward" (2015). Master's Theses. 132.

https://aquila.usm.edu/masters_theses/132

This Masters Thesis is brought to you for free and open access by The Aquila Digital Community. It has been accepted for inclusion in Master's Theses by an authorized administrator of The Aquila Digital Community. For more information, please contact Joshua.Cromwell@usm.edu. 


\title{
ZEBRAFISH AND CONDITIONED PLACE PREFERENCE:
}

A TRANSLATIONAL MODEL OF DRUG REWARD

\author{
by
}

Adam Douglas Collier

\begin{abstract}
A Thesis
Submitted to the Graduate School

of The University of Southern Mississippi

in Partial Fulfillment of the Requirements

for the Degree of Master of Arts
\end{abstract}

Approved:

Dr. David Echevarria, Committee Chair

Associate Professor, Psychology

Dr. Richard Mohn, Committee Member

Associate Professor, Educational Studies and Research

Dr. Brad Dufrene, Committee Member

Associate Professor, Psychology

Dr. Peter McLaughlin, Committee Member

Professor, Psychology, Edinboro University of Pennsylvania

Dr. Karen S. Coats

Dean of the Graduate School 


\section{ABSTRACT \\ ZEBRAFISH AND CONDITIONED PLACE PREFERENCE: \\ A TRANSLATIONAL MODEL OF DRUG REWARD \\ by Adam Douglas Collier}

August 2015

Addiction and substance abuse commonly lead to negative outcomes such as damaged health, domestic violence, child abuse, failure in school, and loss of employment. In the United States, hundreds of billions of dollars accrue annually in costs associated with healthcare, crime and lost productivity due to addiction. Efficacious treatments remain few in number, the development of which will be facilitated by comprehension of environmental, genetic, pharmacological, and neurobiological mechanisms implicated in the pathogenesis of addiction. The zebrafish (Danio rerio) has recently gained popularity as a model organism of complex brain disorders (e.g., substance use disorder). Behavioral quantification within the conditioned place preference (CPP) paradigm serves as a measure of the rewarding qualities of a given stimulus (e.g., drug). If animals develop an increase in preference to spend time in an environment that had previously been paired with drug administration, the drug is inferred to have rewarding properties. This project reports the effects of acute (1 day) and chronic (7 days) exposure to alcohol, caffeine, and nicotine on zebrafish CPP behavior. 


\section{ACKNOWLEDGMENTS}

The author would like to express his gratitude to his thesis committee chair and faculty mentor, Dr. David Echevarria, in addition to the other committee members, Dr.'s Richard Mohn, Brad Dufrene, and Peter McLaughlin, for their constructive feedback and support throughout the duration of this project. I would especially like to thank Dr.

Echevarria for sharing his expertise and providing guidance throughout the duration of my graduate studies. The author also expresses thanks to his colleagues, Kanza Khan, Erika Caramillo, and Natalie Lodinger, for their invaluable help and support throughout this project. 


\section{TABLE OF CONTENTS}

ABSTRACT

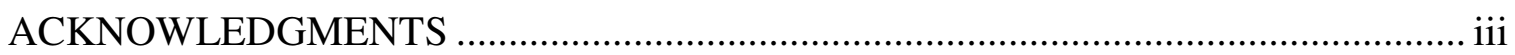

LIST OF TABLES

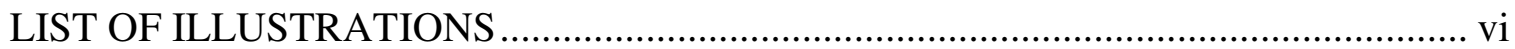

LIST OF ABBREVIATIONS ................................................................................ vii

CHAPTER

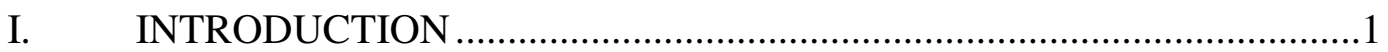

The Zebrafish Model

A Neurobehavioral Model

Conditioned Place Preference (CPP)

The Current Study

Hypotheses and Research Question

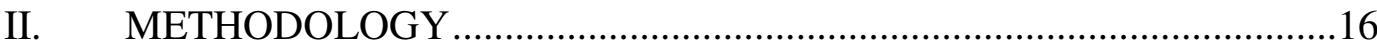

Subjects and Husbandry

The CPP Apparatus

The CPP Procedure

Statistical Analyses

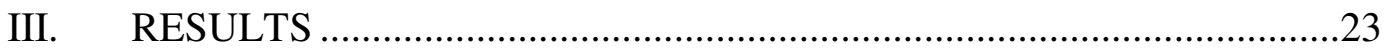

Ethanol CPP Results

Caffeine CPP Results

Nicotine CPP Results

Side Preference and Conditioning Order Results

IV. DISCUSSION

Limitations

Conclusion

APPENDICES

REFERENCES 


\section{LIST OF TABLES}

Table

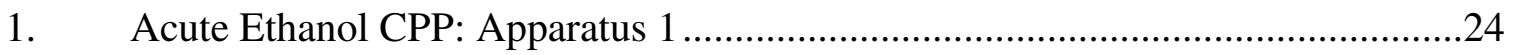

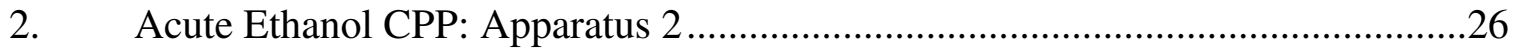

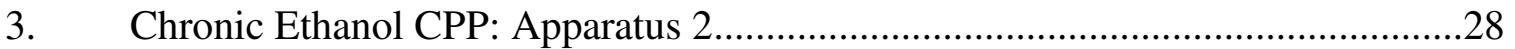

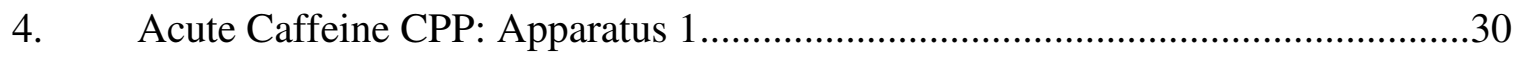

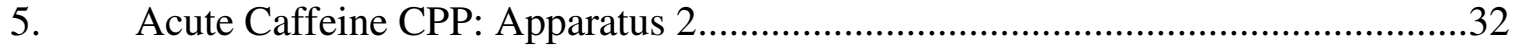

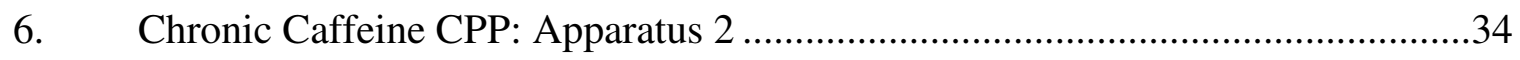








\section{LIST OF ILLUSTRATIONS}

Figure

1. PubMed Search Results ..........................................................................

2. CPP Apparatus Design 1: Preference Testing Apparatus ...................................17

3. CPP Apparatus Design 1: Conditioning Apparatus ..........................................18

4. CPP Apparatus Design 2: Preference Testing Apparatus ..................................18

5. CPP Apparatus Design 2: Conditioning Apparatus ........................................19

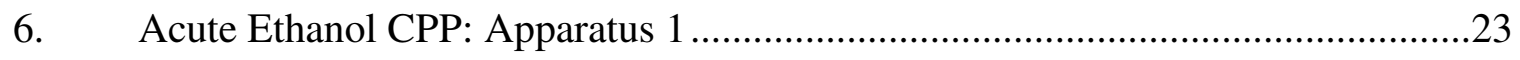

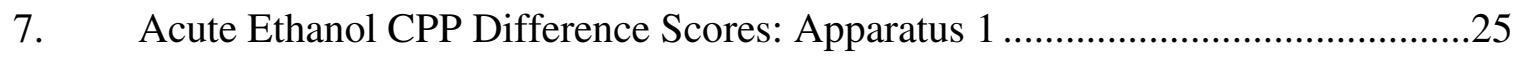

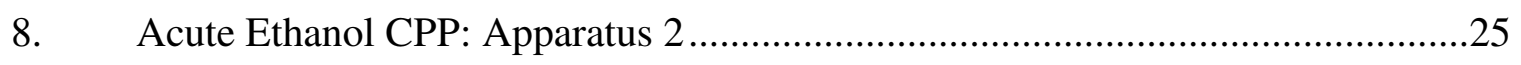

9. Acute Ethanol CPP Difference Scores: Apparatus 2 ….................................27

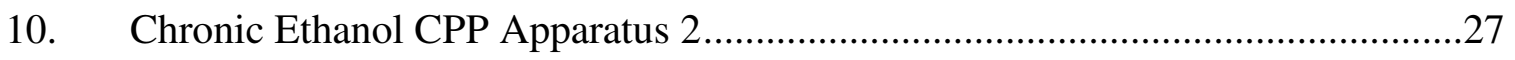

11. Chronic Ethanol CPP Difference Scores: Apparatus 2 ...................................28

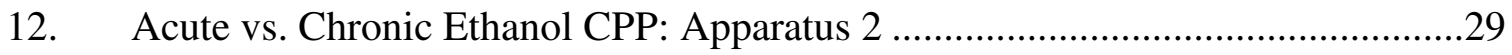

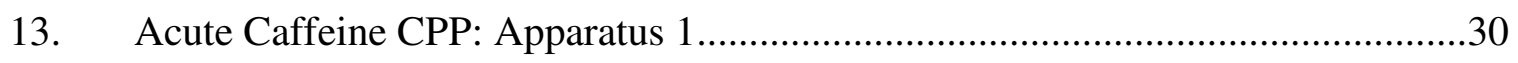



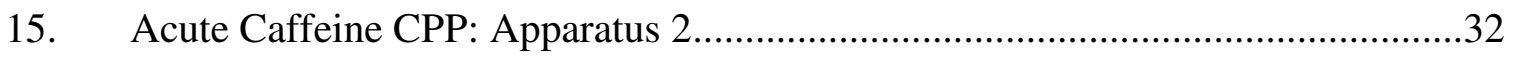

16. Acute Caffeine CPP Difference Scores: Apparatus 1 ......................................33

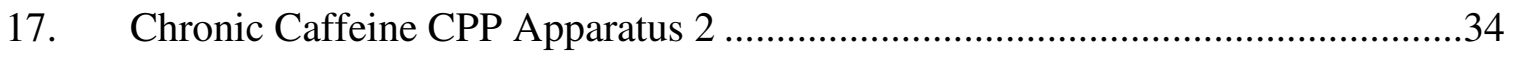

18. Chronic Caffeine CPP Difference Scores: Apparatus 2 ...................................35

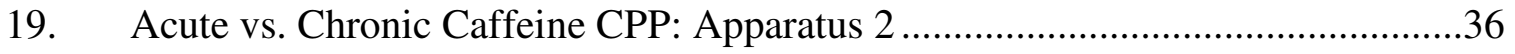

20. Acute Nicotine CPP: Apparatus 2 .............................................................

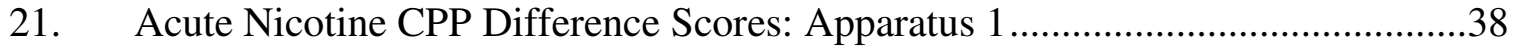




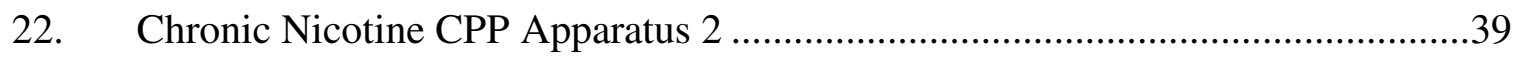

23. Chronic Nicotine CPP Difference Scores: Apparatus 2 ....................................40

24. Acute vs. Chronic Nicotine CPP: Apparatus 2 ............................................. 41

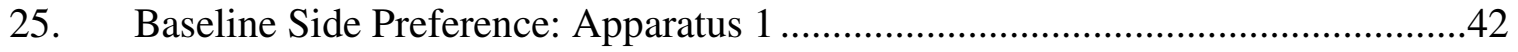

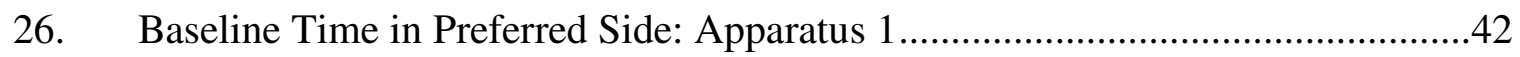

27. Baseline Side Preference: Apparatus 2 ..........................................................43

28. Baseline Time in Preferred Side: Apparatus 2 .............................................44

29. Conditioning Order and Change in Place Preference: Apparatus 2.....................45 


\section{LIST OF ABBREVIATIONS}

$\begin{array}{ll}B A C & \text { Blood Alcohol Concentration } \\ C N S & \text { Central Nervous System } \\ C P P & \text { Conditioned Place Preference } \\ C P A & \text { Conditioned Place Preference } \\ C S & \text { Conditioned Stimulus } \\ \text { DSM } & \text { Diagnostic and Statistical Manual of Mental Disorders } \\ N A c & \text { Nucleus Accumbens } \\ P F C & \text { Prefrontal Cortex } \\ U S & \text { Unconditioned Stimulus } \\ V T A & \text { Ventral Tegmental Area } \\ Z F I N & \text { Zebrafish Information Network } \\ Z N D & \text { Zebrafish Neurophenome Database }\end{array}$




\section{CHAPTER I}

\section{INTRODUCTION}

Substance abuse and addiction are complex and ubiquitous problems; they not only negatively affect individuals, but are a tremendous burden to the global economy as well. Alcohol, nicotine, and caffeine are three substances with widespread availability throughout much of the world, and are thus commonly used by many people. Alcohol is a particularly devastating substance. The consumption of alcoholic beverages is the third largest risk factor for disease in the world, and is responsible for roughly 2.5 million deaths each year (World Health Organization, 2011). Worldwide, the annual consumption of alcohol is estimated to be eight times higher than the annual prevalence of illicit drug use (United Nations Office on Drugs and Crime, 2012). Tobacco use is the number one preventable cause of mortality and morbidity in the United States, and is responsible for about 1 in every 5 deaths (National Institute on Drug Abuse, 2014). Nicotine, an addictive psychoactive alkaloid found in the tobacco plant, is responsible for higher rates of dependence than any other substance of abuse (Centers for Disease Control, 2015). The use of caffeine, a less harmful substance, is not described as having potential to result in a clinically significant use disorder according to the DSM-V. However, caffeine is the most commonly used drug in the world (Winston, 2005) with over $85 \%$ of children and adults consuming it regularly, more than $70 \%$ of which experience at least one withdrawal symptom following cessation of use (American Psychiatric Association, 2013).

The development of novel pharmacotherapies and targeted intervention strategies will be facilitated by comprehension of the various mechanisms (e.g., environmental, 
genetic, pharmacological and neurobiological) implicated in the pathogenesis of addiction. Animal models have often been utilized to help elucidate such mechanisms and processes, most notably those associated with the experience of reward. Animal survival is often dependent upon learning the conditions necessary to acquire naturally rewarding and reinforcing stimuli that serve homeostatic and reproductive purposes (Hyman, Malenka, \& Nestler, 2006). Animals rapidly learn the behavioral responses necessary to obtain natural rewards (e.g., mating opportunities, food and water) and the environmental cues that predict them (Bell, Meerts, \& Sisk, 2010; Lau, Bretaud, Huang, Lin, \& Guo, 2006).

Comparable learning also occurs following consumption of psychoactive substances (Everitt, Dickinson, \& Robbins, 2001; Hyman et al., 2006). Rapid conditioning often takes place when drug use is paired with an environment, object, or emotional state, primarily due to the integrated nature of the brain's reward circuitry with the memory, motivational, and emotional centers of the limbic system (McLellan, Lewis, O'Brien, \& Kleber, 2000). Exposure to a stimulus (e.g., environmental) may induce craving for the drug in individuals that are dependent on a substance, and even in those who have been abstinent from drug use for a period of time, potentially resulting in relapse (Childress et al., 1999).

Understanding how such factors contribute to drug seeking behaviors may facilitate new treatment and prevention strategies. Rats and mice have been conventionally employed in this endeavor, chiefly due to the anatomical, biological, and genomic homology between rodents and humans (Lieschke \& Currie, 2007). However, rodent models are uneconomical, have challenging husbandry, and are not amenable to 
methods of high-throughput screening. The zebrafish (Danio rerio) provides an opportunity to overcome these limitations.

The Zebrafish Model

The zebrafish, belonging to the minnow family, is a small freshwater fish geographically native to the shallow flood-plain waters of north-eastern India, Bangladesh and Myanmar (Engeszer, Patterson, Rao, \& Parichy, 2007; Spence, Gerlach, Lawrence, \& Smith, 2008). Reproduction occurs via spawning; about 100 eggs are released per mating event onto substrate which are then externally fertilized by a male sperm cloud (Ruhl, McRobert, \& Currie, 2009). In laboratory conditions zebrafish will spawn every few days throughout the year, most often occurring after dawn. Zebrafish remain transparent through embryonic and larval stages, hatch 2-3 days post-fertilization, and inflate their gas bladders around day 5 to begin free swimming (Reed \& Jennings, 2010). Basic body architecture develops within 24 hours in this species, equivalent to about 9 days in the mouse (Lardelli, 2000).

Furthermore, zebrafish reach sexual maturity and adulthood in about 3 months, although the rate of individual development may be influenced by environmental and genetic factors (Reed \& Jennings, 2010). The small size of adult zebrafish $(4 \mathrm{~cm}$ long) permits easy handling and the housing of a large number of fish in a small laboratory environment (Pan, Chatterjee, \& Gerlai, 2012). The upsurge in popularity of the zebrafish model over the past several decades has been profound. For example, a PubMed query with the search term 'zebrafish' reveals 86 publications in the year 1993, and 926 publications ten years later in 2003, a 10.8 fold increase (Figure 1). The number of mouse publications in the same period experienced a mere 1.6-fold increase. 


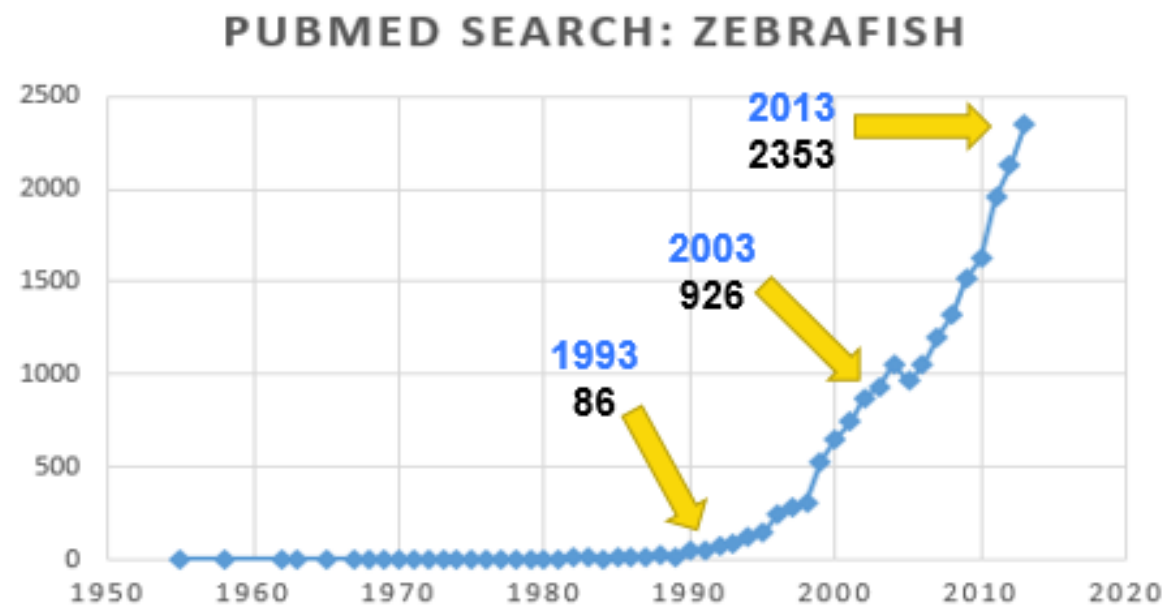

Figure 1. PubMed search results with the search term 'zebrafish'

The capability to observe cell-biological events of early zebrafish development in vivo attracted researchers to adopt this species as an embryological model as early as the 1930s (Lieschke \& Currie, 2007). Throughout the 1980s, new genetic techniques became readily available, such as cloning and mutagenesis, which progressed the use of the zebrafish as a model to investigate genetic components of vertebrate development (Streisinger, Walker, Dower, Knauber, \& Singer, 1981; Walker \& Streisinger, 1983). In 1996, genetic screens identified over 4000 mutations and were published in the journal Development (Driever et al., 1996; Haffter et al., 1996). Recently, sequencing of the zebrafish genome has been completed, and $\sim 70 \%$ of human genes were found to have at least one zebrafish orthologue, with $84 \%$ of genes associated with human disease being present in zebrafish (Howe et al., 2013). This model has been regarded to be particularly ideal for genetic research due to such translational value, high fecundity, rapid development, and amenability to high-throughput screening of genetic mutations and small molecules (Lieschke \& Currie, 2007).

A Neurobehavioral Model 
Zebrafish have recently been adopted as a model to study animal behavior, specifically as it relates to the function and dysfunction of the nervous system. This burgeoning field is augmented by the vast data accumulated from the rodent model; indeed, many behavioral paradigms utilized in rodent research have been aquatically converted to accommodate the zebrafish, including the open field, light-dark, T-maze, social preference, and predator avoidance tests (Cachat et al., 2013; Gerlai, Lee, \& Blaser, 2006; Gould, 2011; Grossman et al., 2010; Kyzar et al., 2012). Recently, a comprehensive glossary consisting of 190 detailed zebrafish behaviors has been compiled, satisfying the necessity for consistent and well-defined terminology in the field (Kalueff et al., 2013). Some relatively complex behaviors zebrafish are capable of include aggression (Echevarria, Hammack, Jouandot, \& Toms, 2010; Gerlai, Lahav, Guo, \& Rosenthal, 2000), anxiety (Egan et al., 2009; Stewart et al., 2011), learning and memory (Colwill, Raymond, Ferreira, \& Escudero, 2005; Sison \& Gerlai, 2010) and most notably, behaviors relevant to addiction (López Patiño, Yu, Yamamoto, \& Zhdanova, 2008; Mathur \& Guo, 2010). These behaviors may be experimentally, genetically and/or pharmacologically manipulated at both larval and adult stages of development (Guo, 2009).

Although there is morphological disparity between zebrafish and humans, comparable features of the central nervous system (CNS) allow for behavioral results to be generalized to mammals (Guo, 2009). The zebrafish CNS contains many of the major neurotransmitter systems found in mammals, including GABA, glutamate, dopamine, norepinephrine, serotonin, histamine, adenosine and acetylcholine (Panula et al., 2010; Maximino et al., 2011). In humans and rodents, the mesolimbic dopamine system, 
primarily consisting of projections from the ventral tegmental area (VTA) to the nucleus accumbens (NAc), prefrontal cortex (PFC), hippocampus and amygdala, is believed to become activated by all drugs of abuse (Koob \& Volkow, 2010). Although this mesolimbic dopamine system is not conserved among humans and zebrafish, the anatomical organization of the nervous system is similar among vertebrates, and the lateral and medial pallium, as well as dopaminergic projections to the zebrafish forebrain, are believed to be homologous to the associated mesolimbic circuitry in mammals (Gould, 2011; Rink \& Wullimann, 2002a, 2002b)

A particularly important brain structure implicated in reward learning is the amgydala. This structure assigns positive or negative value to various stimuli, and becomes activated by drugs of abuse as well as drug-associated cues (Carelli, Williams, \& Hollander, 2003; Paton, Belova, Morrison, \& Salzman, 2006). It has recently been discovered that the amygdala is responsible for integrating both motivational and spatial information (Peck, Lau, \& Salzman, 2013). In the zebrafish brain, the medial pallium has been described as structurally and functionally homologous to the mammalian amygdala. Increased neuronal activation, measured by expression of the immediate early gene cfos, has been reported in this zebrafish brain structure during both conditioned learning and drug seeking behavior (Trotha, Vernier, \& Bally-Cuif, 2014).

In mammals, the hippocampus is largely responsible for spatial memory, and although zebrafish lack this region, the lateral pallium is believed to be structurally homologous, suggesting a conservation of some cognitive processes (Tropepe \& Sive, 2003). The lateral pallium has been found to become activated in zebrafish during a conditioned learning task (Trotha et al., 2014). Zebrafish have been found to be capable 
of completing a variety of cognitive tasks. For example, following the pairing of a visual stimulus (i.e., a red cue card) with a reward (i.e., the sight of a conspecific), it was found that zebrafish would eventually approach the cue card in the absence of the rewarding stimulus, suggesting that zebrafish are capable of forming CS-US associations (Karnik \& Gerlai, 2012). In another study, zebrafish were placed into a tank, half of which was colored white and the other half colored black, and upon each entry into the black half of the tank a mild shock was applied to the water. On the following day, zebrafish were found to display an increased aversion for the black environment and thus suggesting the development of avoidance learning (Manuel et al., 2014)

As a result of the aforementioned behavioral and CNS similarities, the zebrafish has emerged as a promising vertebrate model of a wide range of human domains and disorders, including, but not limited to, depression (Ziv et al., 2013), anxiety behavior (Stewart et al., 2011), social behavior (Echevarria, Buske, Toms, \& Jouandot, 2011; Miller \& Gerlai, 2011), epilepsy (Wong, et al., 2010), sleep disorders (Zhdanova, 2011), and most notably, addiction (Darland \& Dowling, 2001; Stewart et al., 2010; Stewart et al., 2011). Drugs of abuse have been observed to induce tolerance, withdrawal, and place preference in both larval and adult zebrafish (Canavello et al., 2010; Stewart et al., 2011; Tran \& Gerlai, 2013). Adult zebrafish exposed to $0.3 \%$ ethanol, diazepam, or morphine for 2 weeks and then placed in fresh water to simulate drug withdrawal have been reported to display anxiogenic phenotypes and a significant increase in whole-body cortisol levels (Cachat et al., 2010). These results are comparable to the effects of withdrawal on rodent measures of behavior and physiology (Almela et al., 2012; Silva \& Madeira, 2012), indicating good face and construct validity of the zebrafish model 
(Hyman et al., 2006). This proposed study will capitalize on the advantageous and translational characteristics of the zebrafish model of drug reward, facilitated by a wellestablished experimental paradigm for evaluating the rewarding (or aversive) properties of drugs.

\section{Conditioned Place Preference (CPP)}

Since its inception, CPP has been extensively utilized, primarily with rodents, to evaluate rewarding effects of psychoactive compounds (Tzschentke, 1998; Tzschentke, 2007). The apparatus used in CPP testing can vary in design, but typically consists of a conditioning box comprised of two or three distinct environmental compartments (Darland et al., 2012; Kily et al., 2008). In the latter design, a central neutral chamber acts as a starting zone and allows passage between conditioning compartments (Darland et al., 2012; Lau et al., 2006; Mathur, Berberoglu, \& Guo, 2011).

The CPP procedure is generally comprised of three testing phases that occur on consecutive days. During phase 1 the animal is permitted to explore all compartments of the apparatus, and the time spent in each compartment is quantified and used as baseline place preference. In phase 2, animals are sequentially restricted to each compartment for a period of time in which they receive either experimental or control treatment. In phase 3, the animal is once again allowed access to all compartments and final place preference is measured. CPP behavior is typically evaluated by subtracting phase 1 place preference from phase 3 preference (i.e., final place preference - baseline place preference) (Mathur, et al., 2011). This value is used to quantify place preference behavior, and if a significant change towards the experimental compartment is observed, CPP is established, and the experimental treatment is inferred to be rewarding. Conditioned place aversion (CPA) is 
conceptually identical to the CPP test, except in that the experimental treatment exhibits aversive, often unpleasant qualities. If animals avoid the environment in which the treatment was administered, CPA learning has occurred (Braida et al., 2007).

The learning processes necessary to form an association between an environmental stimulus and a drug stimulus are likely to follow the principles of classical (Pavlovian) conditioning. The drug acts as an unconditioned stimulus (US), which elicits a response (e.g., reward) in animals prior to any learning taking place. The environment, which is normally a neutral stimulus on its own, gains incentive salience and becomes a conditioned stimulus (CS) following pairing with the US. The presence of the CS alone elicits a conditioned response of place preference behavior following such pairing. However, this response is differential in quality to that of a classically conditioned response such as the involuntary reflex of salivation in Pavlov's studies with dogs. A conditioned response in CPP involves the behavior of approaching the CS and spending time there.

In operant (respondent) conditioning paradigms, as in drug self-administration, a common alternative to CPP, the presence of the US (e.g., drug) is dependent upon engaging in a behavior, such as lever pressing, and is thus under control of the animal. Self-administration of a drug such as cocaine for example, reinforces a voluntary behavioral response necessary for drug delivery (Goeders \& Guerin, 1996). In contrast, drugs are passively administered by the experimenter in CPP, which is not dependent upon an animal behavior. Therefore, there is no response required from animals to receive the US in CPP testing, unlike in self-administration procedures. Distinct neurochemical differences in the mesolimbic dopamine system have been found in 
animals that have self-administered amphetamine compared to those that received the drug passively (Di Ciano, Blaha, \& Phillips, 1998). Thus, CPP differentially assesses drug reward and engages distinct neuropharmacological circuitry compared to operant procedures such as self-administration (Tzschentke, 1998). For the purposes of this study, the term 'reward' will be used throughout the duration of this document and inferred to be the primary measure of CPP, rather than 'reinforcement'.

An important methodological concern in CPP studies is the whether the apparatus is 'biased' or 'unbiased'. The CPP apparatus may be designed in such a way that animals will reliably display place preference for one environment over the other prior to conditioning, and is referred to as a biased design (Tzschentke, 2007). In an unbiased design, animals do not display a strong preference for one environment over the other before conditioning takes place. The effect of biased and unbiased apparatus design has been investigated in ethanol place conditioning in mice (Cunningham, Ferree, \& Howard, 2003). Both designs were employed, and in each, ethanol was randomly paired with environmental stimuli such that animals received ethanol in initially preferred and initially non-preferred environments. CPP was observed with the unbiased apparatus regardless of ethanol being paired with the preferred or non-preferred side. Yet, CPP was only observed when ethanol was paired with the non-preferred side with the biased apparatus. Thus, apparatus design is of notable concern when evaluating the rewarding or aversive effects of novel compounds. As a result, the unbiased design has been predominately employed and held in higher regard than the biased design (SanchisSegura \& Spanagel, 2006). 
Both designs have been employed in zebrafish CPP literature, although the majority of which have been unbiased. An innate preference bias towards brown CPP environments over light environments with two black spots has been observed in zebrafish (Kedikian, Faillace, \& Bernabeu, 2013; Ninkovic \& Bally-Cuif, 2006; Ninkovic et al., 2006), and nicotine CPP has been reported using both unbiased and biased designs (Kedikian et al., 2013; Kily et al., 2008). There are several reviews available that comprehensively catalogue the CPP literature in detail, including discussion of the aforementioned issues pertinent to methodology and design (Schechter \& Calcagnetti, 1998; Tzschentke, 1998; Bardo \& Bevins, 2000; Tzschentke, 2007, Collier \& Echevarria, 2013, Collier, Khan, Caramillo, Mohn, \& Echevarria, 2014).

In order for animal CPP studies to have good face validity and to contribute to the endeavor of reducing suffering related to pandemic drug abuse, the results must be relevant to humans. Childs \& Wit (2009) treated human participants with either damphetamine or placebo within two distinct environments, and found that people preferred the place associated with amphetamine treatment. In another human CPP study, the researchers used music as US and utilized several virtual reality environments to serve as CS. Half of the participants were asked to visit a virtual house that played consonant music for two minutes, and then visited another virtual house that played static noise for two minutes, and the remaining half visited the environments in the reverse order. After conditioning took place the participants were free to spend time in either of the two houses, and it was found that subjects displayed CPP towards the house with the consonant music (Molet, Billiet, \& Bardo, 2013). Thus, like laboratory animals, humans implicitly learn associations between environmental stimuli and direct experience. 
The degree of reward experienced from a drug experience is suggested to predict the potential of that substance to be abused (Haertzen et al., 1983). CPP has been induced in the rodent model by addictive substances frequently abused by humans, including d-amphetamine (Yates, Marusich, Gipson, Beckmann, \& Bardo, 2012), cocaine (Bahi, Kusnecov, \& Dreyer, 2008; Russo et al., 2008), diazepam (Papp, Gruca, \& Willner, 2002), ethanol (Kotlinska, Bochenski, \& Danysz, 2011), heroin (Braida, Pozzi, Cavallini, \& Sala, 2001), ketamine (Li et al., 2008), methamphetamine (Zakharova, Leoni, Kichko, \& Izenwasser, 2009), morphine (Liang et al., 2006), and nicotine (Brielmaier, McDonald, \& Smith, 2008). The literature reveals that CPP has not been established with drugs that humans do not typically abuse, such as antidepressants, neuroleptics, and antihistamines, which is indicative of construct validity of the CPP assay (Papp et al., 2002).

Comparable to many rodent behavioral paradigms, CPP has recently been adopted in zebrafish neurobehavioral research (Darland \& Dowling 2001; Ninkovic \& Bally-Cuif 2006; Mathur et al. 2011b; Parmar et al. 2011). Various drugs have been observed to induce CPP behavior in zebrafish, often following a single administration, demonstrating the potent rewarding properties of these substances and validating the translational value of the zebrafish CPP model of drug reward. For example, zebrafish have been reported to develop CPP towards amphetamine (Ninkovic et al., 2006), cocaine (Darland \& Dowling, 2001; Darland et al., 2012), ethanol (Mathur, Berberoglu, et al., 2011), morphine (Lau et al., 2006), salvinorin A (Braida et al., 2007), and nicotine (Bernabeu, Aires, \& Behavior, 2013). CPP is a relatively simple and inexpensive experiment, and 
when coupled with the zebrafish model, experimental protocols may be automated with multiple fish being simultaneously tested (Mathur et al., 2011).

\section{The Current Study}

Substance abuse is a significant public health concern with detrimental consequences, both domestically and worldwide. Comprehending the relationship between drug exposure and conditioning may facilitate the development of new preventative strategies and treatments. For example, a better understanding of how environmental factors contribute to drug seeking behavior and relapse may increase the efficacy of cognitive-behavioral models, such as relapse prevention, by identifying highrisk situations for clients (Larimer, Palmer, \& Marlatt, 1999; Marlatt \& Donovan, 2005). In this endeavor, behavioral research with the zebrafish model and CPP assay may yield significant insight into the relationship between drug reward and learning.

Zebrafish are a relatively new model in the field of behavioral pharmacology. As a result, there is a shortage of associated background literature, especially in comparison to the well-established and data abundant rodent model. Place preference behavior in zebrafish has been defined as "the tendency to establish a preferred location in which the fish spends more time. Can be induced by drugs, repeated administration of food/food odors, social reward, or be based on natural behaviors or preferences" (Kalueff et al., 2013). The current study investigated the effects of ethanol, caffeine, and nicotine on place preference behavior in zebrafish. Ethanol and nicotine zebrafish CPP behavior have been reported, although only a limited range of doses and durations of exposure have been tested (Kedikian et al., 2013; Kily et al., 2008; Mathur, Lau, et al., 2011; Parmar, Parmar, \& Brennan, 2011). For example, nicotine CPP has only been 
investigated following one and three conditioning sessions, and a mere three doses of ethanol have been evaluated in the zebrafish CPP literature. Caffeine CPP in zebrafish has not been reported at any concentration. Thus, the caffeine findings in this study are novel.

There is a crucial need for the investigation of a broader range of doses and durations of exposure to the aforementioned substances in an effort to better establish the zebrafish model of drug reward. The current study was inspired by this rationale. Zebrafish place preference behavior following acute administration (i.e., one conditioning session) of four separate doses of ethanol (i.e., $0.00 \%, 0.25 \%, 0.50 \%$, and $1.00 \%$ ), four doses of caffeine (i.e., $0 \mathrm{mg} / \mathrm{L}, 50 \mathrm{mg} / \mathrm{L}, 100 \mathrm{mg} / \mathrm{L}$ and $150 \mathrm{mg} / \mathrm{L}$ ), and four doses of nicotine (i.e., $0 \mathrm{mg} / \mathrm{L}, 2.5 \mathrm{mg} / \mathrm{L}, 5 \mathrm{mg} / \mathrm{L}$ and $10 \mathrm{mg} / \mathrm{L}$ ) was evaluated in two experimental apparatus designs. Zebrafish place preference behavior following chronic administration (i.e., seven conditioning sessions) of the aforementioned drugs and doses was also investigated.

\section{Hypotheses and Research Question}

\section{Hypotheses}

$\mathrm{H}_{1}$ - It was expected that zebrafish would display CPP behavior in a drug and dose and duration (acute vs. chronic) dependent manner, following administration of ethanol, caffeine, and nicotine.

$\mathrm{H}_{2}$ - It was expected that an equal number of zebrafish would display a baseline place preference for each environment, of both apparatus designs, and an equal number of time would be spent in each environment during baseline place preference testing. 
$\mathrm{H}_{3}$ - It was expected that conditioning order (i.e., receiving drug first or second) would have an effect on place preference behavior.

Research Question

The researcher seeks to determine if the zebrafish model organism, coupled with the CPP assay, may be employed as an effective and valid model of drug reward. 


\section{CHAPTER II}

\section{METHODOLOGY}

\section{Subjects and Husbandry}

All fish were maintained and protocols carried out according to the Institutional Animal Care and Use Committee of the University of Southern Mississippi, Hattiesburg MS, USA. Adult zebrafish of a randomly bred genetically heterogeneous 'wildtype' strain were obtained from a local distributor (Pet Palace, Hattiesburg MS 39401). All fish were acclimated to the laboratory environment for a minimum of 10 days, housed within a $55 \mathrm{~L}$ ( $76 \mathrm{~cm}$ high $\times 30 \mathrm{~cm}$ wide $\times 25 \mathrm{~cm}$ high) group holding tank, and then individually and adjacently housed within $2.5 \mathrm{~L}$ tanks $(20 \mathrm{~cm}$ high x $13 \mathrm{~cm}$ long x $14 \mathrm{~cm}$ high) at least 48 hours prior to behavioral testing. All tanks were maintained in a circulating system equipped with biological, chemical, and mechanical filtration, aeration, and sterilization by UV light. Ceiling-mounted fluorescent light tubes provided illumination during a 14/10 hour light/dark cycle. Tank water consisted of reverse osmosis deionized H2O supplemented with $60 \mathrm{mg} / \mathrm{L}$ dissolved sea salts (Instant Ocean: Blacksburg, VA 24060), and was maintained at $\sim 25-27 \mathrm{C}^{\circ}$. Fish were fed once in the morning with brine shrimp (Premium Grade Brine Shrimp Eggs, Brine Shrimp Direct, Ogden, UT), and once in the afternoon with flake food (Tetra: Blacksburg, VA). All animals were drug and experimentally naïve prior to testing. Experimentation took place between 9:00 AM and 4:00 PM. All behavior was recorded by USB webcams (saved as MP4 files for subsequent analysis) mounted to an overhead shelter, which also provided equal light distribution and prevented fish from observing outside the tank. 


\section{The CPP Apparatus}

\section{CPP Apparatus Design 1}

The first CPP apparatus design consisted of a $30 \mathrm{~L}$ glass aquarium $(41 \mathrm{~cm}$ long $\mathrm{x}$ $20 \mathrm{~cm}$ wide $\mathrm{x} 24 \mathrm{~cm}$ tall). Separate aquarium tanks were used for preference testing and conditioning to prevent cross-contamination, and were rinsed with deionized water before and after testing. The preference testing tank was colored by adhesive shelf liner that divided it into two distinct halves; one half was colored white, the other was white with a fixed pattern of 14 black dots $2.43 \mathrm{~cm}$ in diameter (Figure 2) This apparatus design was adopted from previous methods (Mathur et al., 2011)

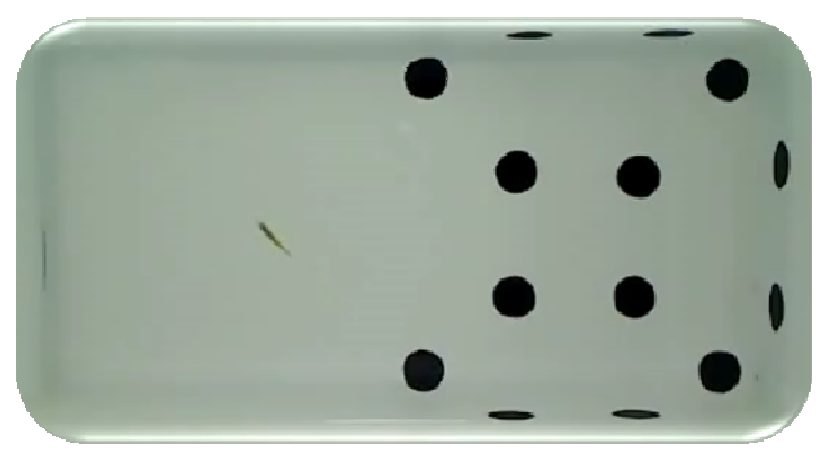

Figure 2. CPP apparatus design 1: Preference testing apparatus

Tanks used for drug administration (i.e., conditioning) were identical in design, with the exception of central divider that was sealed with aquarium sealant to prevent transference of water or drug between chambers. The divider also included an additional two dots, for a total of 16 dots on one side of the conditioning apparatus (Figure 3). 


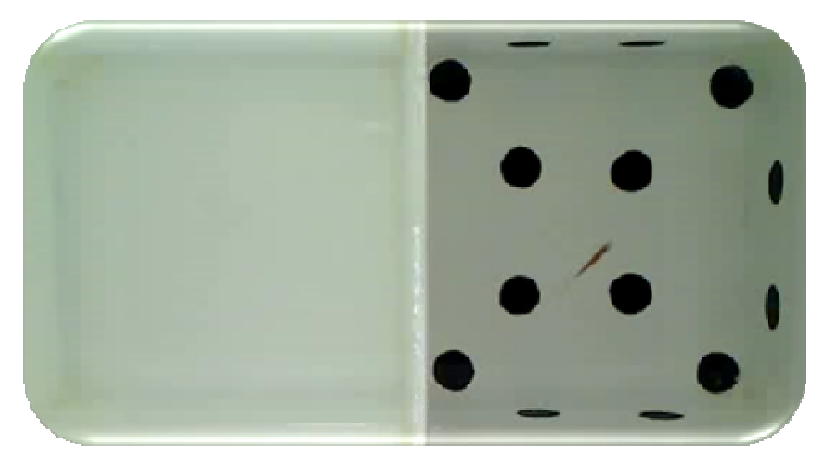

Figure 3. CPP apparatus design 1: Conditioning apparatus

\section{CPP Apparatus Design 2}

The second CPP apparatus design also consisted primarily of a $30 \mathrm{~L}$ glass aquarium (41 cm long x $20 \mathrm{~cm}$ wide $\mathrm{x} 24 \mathrm{~cm}$ tall). The preference testing tank was colored by adhesive shelf liner that divided it into two distinct halves; one half was colored white with a fixed pattern of 15 blue dots, the other was white with a fixed pattern of 15 blue rectangles (Figure 4). The color of the shape patterns were changed from black to blue to create better contrast between zebrafish and the background to aid in video analyses. In an effort to reduce potential bias towards one environment over the other, it was decided to design this apparatus with two similar, but distinct environments. These environments differed in their pattern shapes and pattern design, and were similar in that the shapes were the same color (i.e., blue) and of the same quantity.

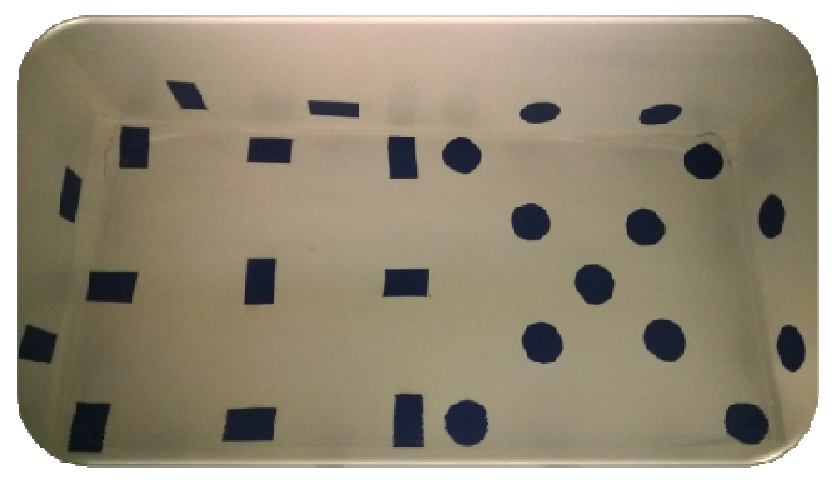

Figure 4. CPP apparatus design 2: Preference testing apparatus 
The second CPP conditioning apparatus differed from the first. In an effort to create a more efficient design and conserve the quantity of drugs used during experimentation, $500 \mathrm{~mL}$ crystallizing dishes colored with adhesive shelf liner were employed as conditioning chambers (Figure 5). Each conditioning dish had a total of 13 shapes, of either dots or rectangles, with patterns that closely mimicked that of the preference testing apparatus.

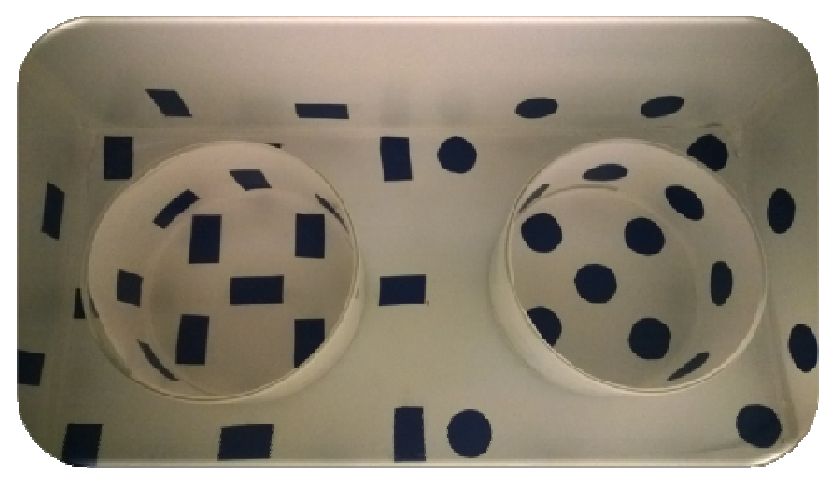

Figure 5. CPP apparatus design 2: Conditioning apparatus

The CPP Procedure

\section{Phase 1: Baseline place preference}

During phase 1, on the first day of experimentation for each cohort, animals were carefully transported, while still within their home tanks, from the housing system to the nearby experimental table. The experimenter then left and allowed zebrafish 10 minutes to acclimate to the new environment. CPP preference testing tanks, in both designs, were filled with $5 \mathrm{~L}$ of system water upon the experimenter's re-entry; fish were then carefully netted from their home tanks and placed directly into the center of the CPP apparatus. Home tanks of animals were close in proximity to the CPP tanks to minimize netting stress and hypoxia. The experimenter quietly left the room and allowed the zebrafish to explore the apparatus for 15 minutes. Fish were then returned to their home tank and 
placed back into the housing system. The initial 5 minutes of exploration were designated for acclimation to the new environment; behavior during this period was not included for analyses.

The duration of time zebrafish spent within each distinct environment was manually recorded via visual observation of video playback by multiple observers for the remaining 10 minutes of exploration. Video analyses occurred in a separate room adjacent to the experimental environment. The times spent in each side of the preference testing tank were then expressed as percentages of the 10 minute testing period, and served as baseline place preference values. Zebrafish that spent $80 \%$ of time or more in one environment were excluded from further testing. Thus, animals that spent between $50.1 \%$ and $79.9 \%$ in one environment were included for the remainder of the experiment, with this environment being deemed as the preferred side, and the remaining environment being labeled as the non-preferred side.

\section{Phase 2: Conditioning}

Following establishment of baseline place preference, each animal was assigned to receive treatment in the non-preferred side. This assay employed a balanced design, in that the order of conditioning was sequenced so that half of the animals were first exposed to treatment and then system water, and the other half were first exposed to system water and then treatment. Previous researchers have reported this balanced order of conditioning to have no significant effect on place preference behavior (Mathur et al., 2011). System water (e.g., $2.5 \mathrm{~L}$ in apparatus design 1 and $0.5 \mathrm{~L}$ in apparatus design 2) was added to each compartment, and appropriate drug concentrations were prepared and dissolved into the water. 
After being transferred and acclimated to the experimental table, fish were netted and placed into the applicable conditioning compartment and allowed to swim freely for 20 minutes. Animals were then netted and placed in a tank containing $1.5 \mathrm{~L}$ system water for 1 minute to wash off any externally bound drug. Lastly, fish were netted and placed into the remaining compartment and once again allowed to explore for 20 minutes. Animals were then removed from the conditioning apparatus, placed in a $1.5 \mathrm{~L}$ tank of system water for 5 minutes, and then returned to home tanks and the housing system. Zebrafish that were conditioned for one day belonged to the acute treatment group, and fish that were conditioned for seven days belonged to the chronic treatment group. During conditioning, experimental animals were treated with a dose of either ethanol (Decon Laboratories, Inc. King of Prussia, PA 19406), anhydrous caffeine (Fisher Scientific, Fair Lawn NJ 07410), or of liquid nicotine (Sigma-Aldrich, St. Louis, MO). Control fish always received system water in the same volume as drug additions. .

\section{Phase 3: Final Place Preference}

On the final day of testing, fish were evaluated for final preference using identical procedures used to determine baseline preference during phase 1. Change in place preference was calculated by subtracting the percentage of time spent in the drug-paired environment before conditioning from the percentage of time in the drug-paired environment after conditioning, and then expressed as a percentage.

\section{Statistical Analyses}

CPP data was first assessed to evaluate changes in place preference for the treatment side before and after conditioning for each drug, dose, and apparatus design by a two-way mixed model ANOVA of drug x time (before conditioning vs. after 
conditioning). The accepted level of significance for ANOVA was $p<0.05$. Pairedsamples post hoc t-tests were used to explore the interaction and evaluate significant differences between place preference for the drug paired side before and after conditioning for each cohort using a Bonferroni adjusted alpha level of $p<0.0125$ (i.e., 05/4). Changes in place preference towards the drug-paired side were compared between groups by a one-way ANOVA, followed by planned comparisons of comparing control to the three doses within each drug cohort. The effect of duration of treatment (i.e., acute vs. chronic) was evaluated for each drug and dose tested from apparatus design 2 by a factorial between-subjects ANOVA followed by simple effects analyses. The effect of environment on time spent in the preferred side during baseline preference testing was assessed using independent measures t-test. The effect of conditioning order on change in preference towards the drug paired side was evaluated with independent measures ttest. SPSS version 23 (IBM Corp, Armonk, NY) was used to perform statistical analyses. CPP data were expressed as mean $( \pm$ SEM). 


\section{CHAPTER III}

\section{RESULTS}

\section{Ethanol CPP Results}

Results revealed a significant main effect of time (i.e., before conditioning vs. after conditioning) on dose of acute ethanol in apparatus $1, F(1,56)=27.603, p<.001$, $\eta p^{2}=.330$. A significant interaction of time and ethanol was not revealed, although this value was approaching statistical significance $F(3,56)=2.620, \eta \mathrm{p}^{2}=.123, \mathrm{p}=0.060$. Post-hoc paired samples t tests revealed significant differences in place preference before and after conditioning for $0.25 \%, 0.50 \%$ and $1.00 \%$ ethanol, indicating that these doses of ethanol induced CPP behavior in zebrafish following a single pairing (see Figure 6 and

\section{Table 1).}



Figure 6. Acute ethanol apparatus 1 CPP behavior: paired samples analyses of time in treatment side before vs. after conditioning data expressed as mean $( \pm \mathrm{SEM}) . * p<0.0125$. 
Table 1

Acute Ethanol Apparatus 1 CPP Behavior: Paired Samples Analyses of Time in Treatment Side Before vs. After Conditioning

\begin{tabular}{|c|c|c|c|c|c|c|c|c|}
\hline \multirow[b]{2}{*}{ Dose } & \multicolumn{2}{|c|}{ Before } & \multicolumn{2}{|c|}{ After } & \multirow[b]{2}{*}{$n$} & \multirow[b]{2}{*}{$t$} & \multirow[b]{2}{*}{$p$} & \multirow[b]{2}{*}{$d$} \\
\hline & $M$ & $S D$ & $M$ & $S D$ & & & & \\
\hline $0.00 \%$ & 37.47 & 9.13 & 39.93 & 9.48 & 15 & 1.29 & 0.215 & 0.28 \\
\hline $0.25 \%$ & 39.60 & 5.54 & 48.13 & 7.86 & 15 & 3.49 & $0.004 *$ & 1.25 \\
\hline $0.50 \%$ & 34.80 & 10.56 & 49.40 & 14.47 & 15 & 2.90 & $0.011 *$ & 1.15 \\
\hline $1.00 \%$ & 36.20 & 9.05 & 53.47 & 18.46 & 15 & 3.06 & $0.008 *$ & 1.88 \\
\hline
\end{tabular}

Note. Statistical significance was determined by a Bonferroni adjusted alpha level of $.05 / 4=0.0125$.

$* \mathrm{p}<.0125$

Planned contrasts revealed that the change in place preference towards the drugpaired side was significantly greater for the group that received acute $0.50 \%$ ethanol compared to the group that received $0.00 \%$ ethanol (i.e., the control group), $t(56)=2.081$, $p=0.041, d=0.82$ (Figure 7). The group that received the highest dose of ethanol, $1.00 \%$, also displayed a significantly greater place preference change towards the drugpaired environment than the control group, $t(56)=2.543, p=0.014, d=0.90$. No significant difference between $0.00 \%$ ethanol and $0.25 \%$ ethanol was revealed. 


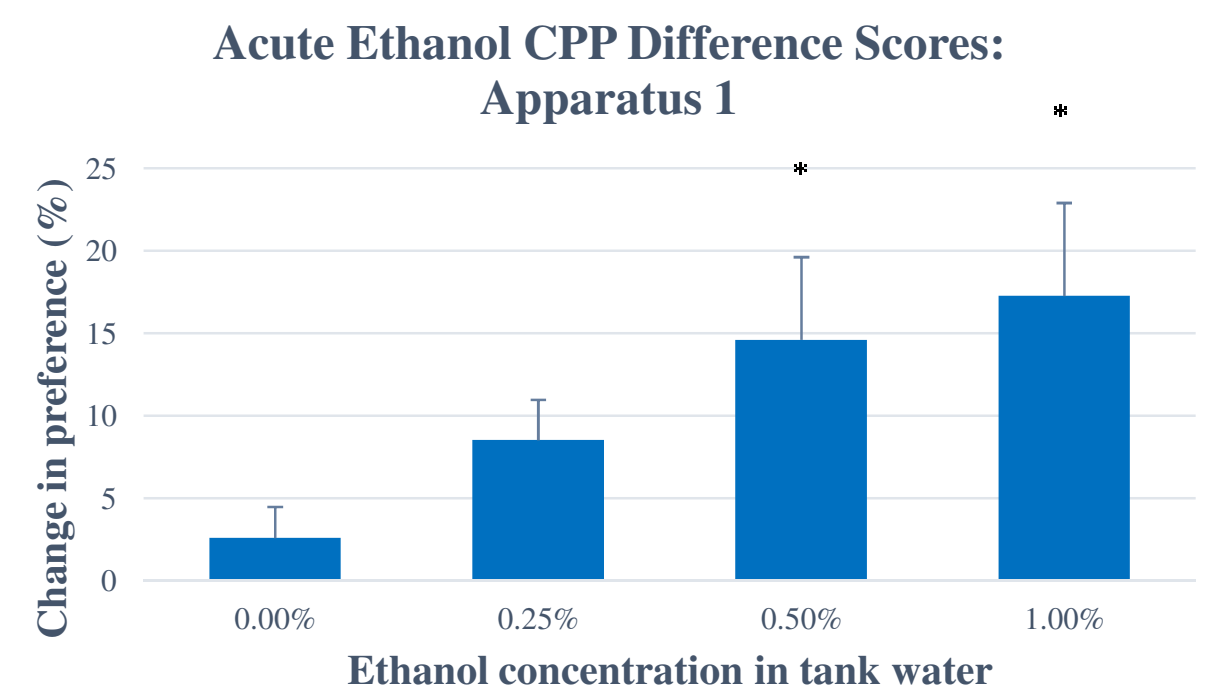

Figure 7. Acute ethanol apparatus 1 CPP difference scores: Change in place preference towards the drug-paired side. Data expressed as mean $( \pm \mathrm{SEM}) . * p<0.05 . * * p<0.01$

Results revealed a significant main effect of time on dose of acute ethanol in apparatus $2, \mathrm{~F}(1,38)=27.55, \mathrm{p}<0.001, \eta \mathrm{p}^{2}=0.42$. A significant interaction of time and ethanol was not revealed, $F(3,38)=1.86, p=0.153, \eta p^{2}=0.13$. Post-hoc paired samples $\mathrm{t}$ tests did not reveal any significant differences in place preference before and after conditioning for $0.25 \%, 0.50 \%$ and $1.00 \%$ ethanol (see Figure 8 and Table 2).

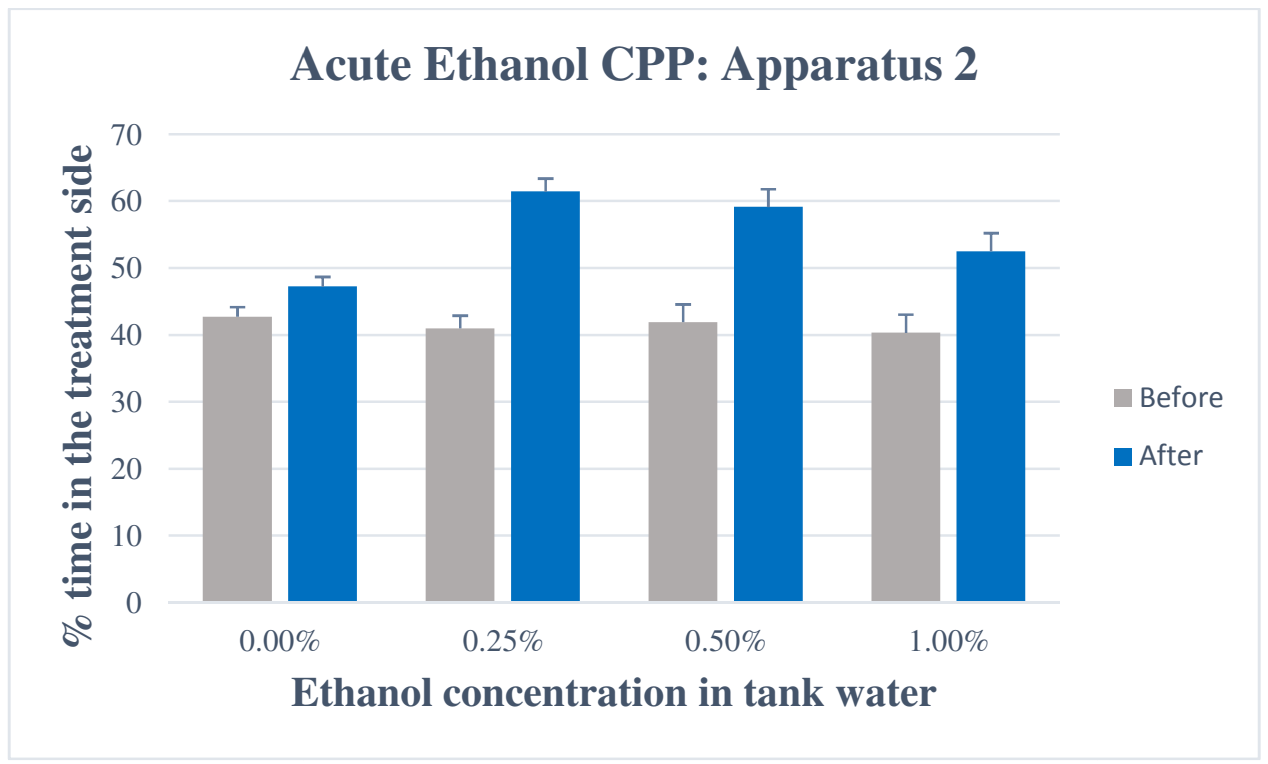


Figure 8. Acute ethanol apparatus 2 CPP behavior: Paired samples analyses of time in treatment side before vs. after conditioning data expressed as mean $( \pm$ SEM $) .{ }^{*} p<0.0125$

Table 2

Acute Ethanol Apparatus 2: Paired Samples Analyses of Time in Treatment Side Before vs. After Conditioning

\begin{tabular}{|c|c|c|c|c|c|c|c|c|}
\hline \multirow[b]{2}{*}{ Dose } & \multicolumn{2}{|c|}{ Before } & \multicolumn{2}{|c|}{ After } & \multirow[b]{2}{*}{$n$} & \multirow[b]{2}{*}{$t$} & \multirow[b]{2}{*}{$p$} & \multirow[b]{2}{*}{$d$} \\
\hline & $M$ & $S D$ & $M$ & $S D$ & & & & \\
\hline $0.00 \%$ & 42.72 & 4.96 & 47.25 & 10.19 & 12 & 1.51 & 0.159 & 0.57 \\
\hline $0.25 \%$ & 40.45 & 4.99 & 60.87 & 22.96 & 9 & 2.99 & 0.014 & 1.20 \\
\hline $0.50 \%$ & 41.94 & 7.45 & 59.14 & 15.37 & 8 & 2.66 & 0.033 & 1.40 \\
\hline $1.00 \%$ & 40.32 & 9.86 & 52.48 & 15.09 & 13 & 2.77 & 0.017 & 0.95 \\
\hline
\end{tabular}

Note. Statistical significance was determined by a Bonferroni adjusted alpha level of $.05 / 4=0.0125$.

$* p<.0125$

Planned contrasts revealed that the change in place preference towards the drugpaired side was significantly greater for the group that received acute $0.25 \%$ ethanol compared to the group that received $0.00 \%$ ethanol (i.e., the control group), $t(38)=2.197$, $p=0.034, d=0.94$ (Figure 9). No significant changes in place preference towards the ethanol-paired side were found between either acute $0.50 \%$ or $1.00 \%$ ethanol groups when compared to the control group. 


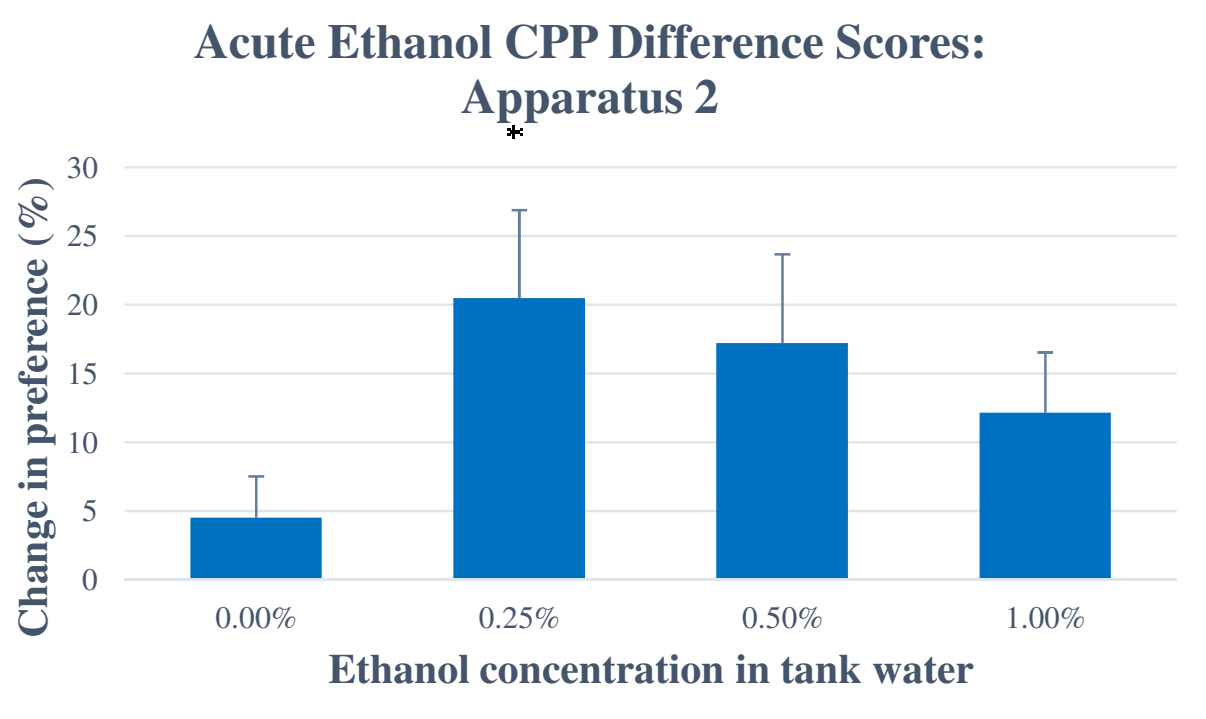

Figure 9. Acute ethanol apparatus 2 CPP difference scores: Change in place preference towards the drug-paired side. Data expressed as mean $( \pm \mathrm{SEM}) .{ }^{*} p<0.05 .{ }^{*} p<0.01$

Results did not reveal a significant main effect of time on dose of chronic ethanol in apparatus $2, \mathrm{~F}(1,25)=5.766, \mathrm{p}=0.24, \eta \mathrm{p}^{2}=0.19$. A significant interaction of time and ethanol was not revealed, $\mathrm{F}(3,25)=0.71, \mathrm{p}=0.557, \eta \mathrm{p}^{2}=0.08$. Post-hoc paired samples t tests did not reveal any significant differences in place preference before and after conditioning for any dose of ethanol (see Figure 10 and Table 3).

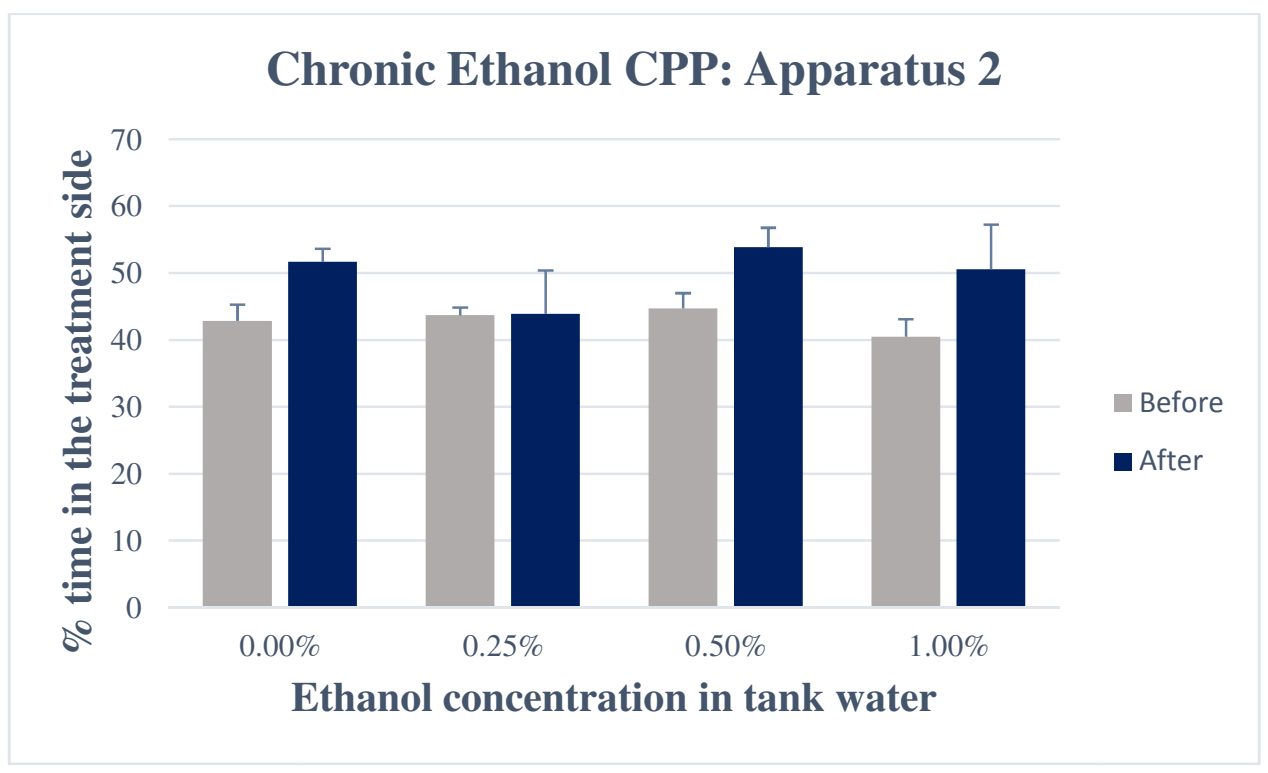


Figure 10. Chronic ethanol apparatus 2 CPP behavior: Paired samples analyses of time in treatment side before vs. after conditioning data expressed as mean $( \pm$ SEM $)$. ${ }^{*} p<0.0125$

Table 3

Chronic Ethanol Apparatus 2: Paired Samples Analyses of Time in Treatment Side Before vs. After Conditioning

\begin{tabular}{|c|c|c|c|c|c|c|c|c|}
\hline \multirow[b]{2}{*}{ Dose } & \multicolumn{2}{|c|}{ Before } & \multicolumn{2}{|c|}{ After } & \multirow[b]{2}{*}{$n$} & \multirow[b]{2}{*}{$t$} & \multirow[b]{2}{*}{$p$} & \multirow[b]{2}{*}{$d$} \\
\hline & $M$ & $S D$ & $M$ & $S D$ & & & & \\
\hline $0.00 \%$ & 42.85 & 6.89 & 51.66 & 5.59 & 8 & 3.05 & 0.019 & 1.40 \\
\hline $0.25 \%$ & 43.69 & 3.26 & 43.90 & 18.36 & 8 & 0.04 & 0.973 & 0.02 \\
\hline $0.50 \%$ & 44.73 & 6.04 & 53.89 & 7.65 & 7 & 2.18 & 0.072 & 1.33 \\
\hline $1.00 \%$ & 40.50 & 6.44 & 50.58 & 16.30 & 6 & 1.21 & 0.279 & 0.81 \\
\hline
\end{tabular}

Note. Statistical significance was determined by a Bonferroni adjusted alpha level of $.05 / 4=0.0125$.

$* \mathrm{p}<.05 . * * \mathrm{p}<.0125$

Planned contrasts revealed that the change in place preference towards the drugpaired side was not significantly different for either chronic $0.25 \%, 0.50 \%$, or $1.00 \%$ ethanol in apparatus 2 when compared to the change in place preference in control fish (Figure 11).

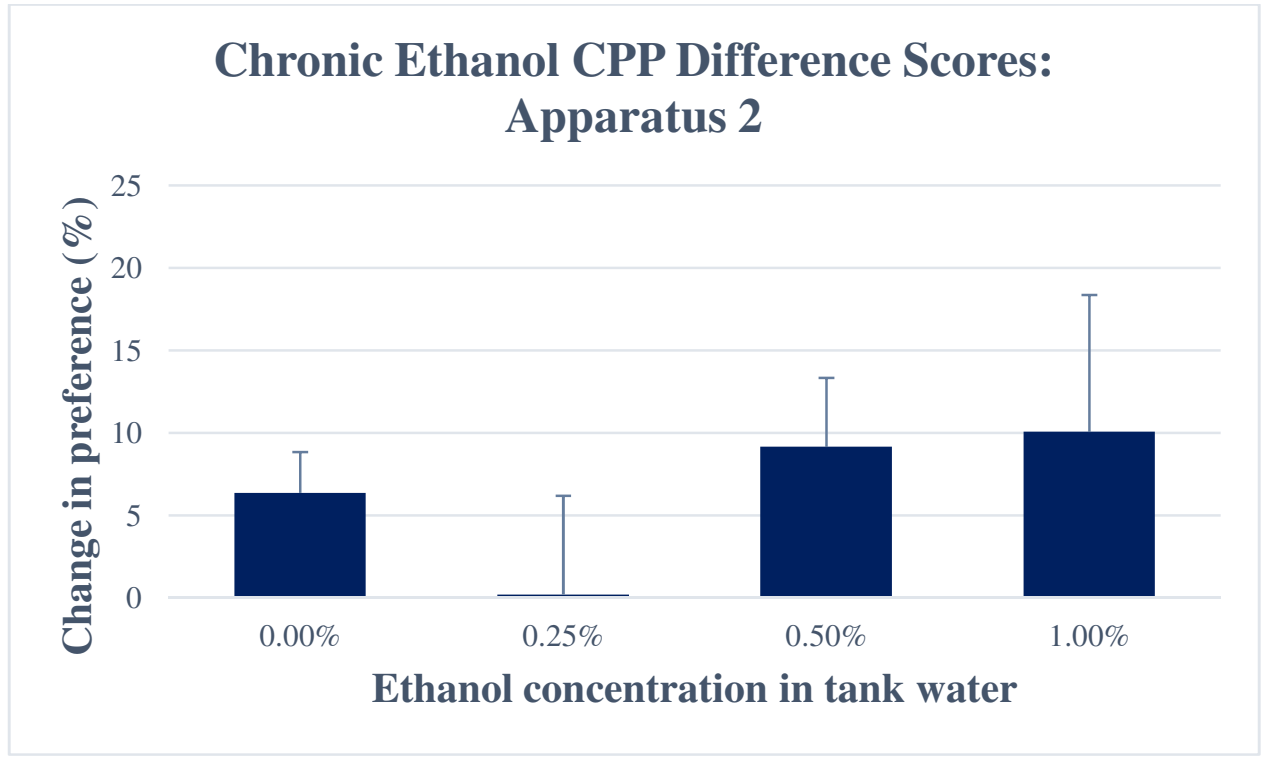


Figure 11. Chronic ethanol apparatus 2 CPP difference scores: Change in place preference towards the drug-paired side. Data expressed as mean $( \pm \mathrm{SEM}){ }^{*} p<0.05 . * * p$ $<0.01$

The effect of duration of treatment (i.e., acute vs. chronic) on change in place preference towards the ethanol paired side was found to be marginally significant, $F(1$, $63)=3.474, p=0.067, \eta p^{2}=0.052$. An analysis of simple effects showed that change in place preference towards the drug-paired side was significantly less for zebrafish treated chronically with $0.25 \%$ ethanol compared to zebrafish treated acutely with $0.25 \%$ ethanol $F(1,63)=7.089, p=0.010, \eta \mathrm{p}^{2}=0.10$. No other significant effects were revealed (Figure 12).

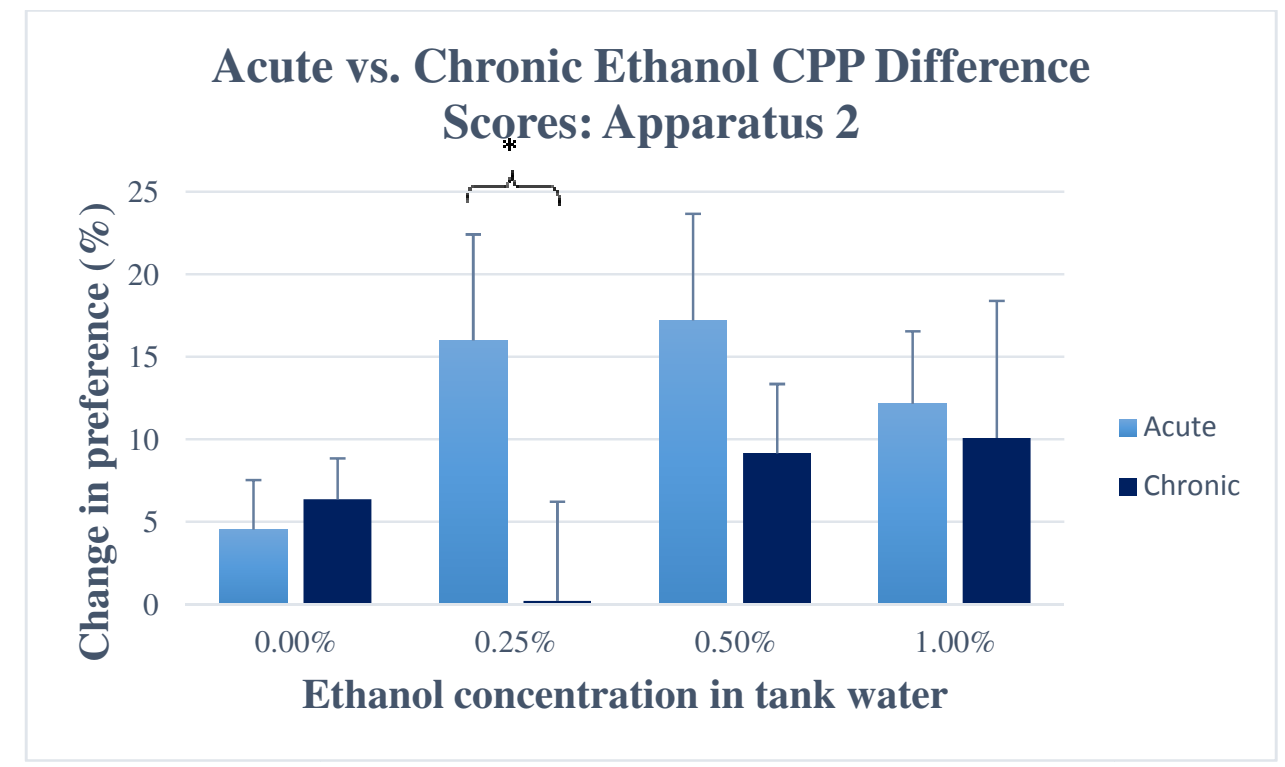

Figure 12. Acute vs. chronic ethanol CPP difference scores: Data expressed as mean $( \pm$ SEM). $* p<0.05 . * * p<0.01$

\section{Caffeine CPP Results}

Results revealed a significant main effect of time (i.e., before conditioning vs. after conditioning) on dose of acute caffeine in apparatus $1, F(1,59)=14.72$, p $<0.001$, $\eta p^{2}=0.20$. A significant interaction of time and caffeine was not revealed, $F(3,59)=$ $0.35, p=0.793, \eta p^{2}=0.02$. Post-hoc paired samples t tests did not reveal any significant 
differences in place preference before and after conditioning for any dose of caffeine (see Figure 13 and Table 4).

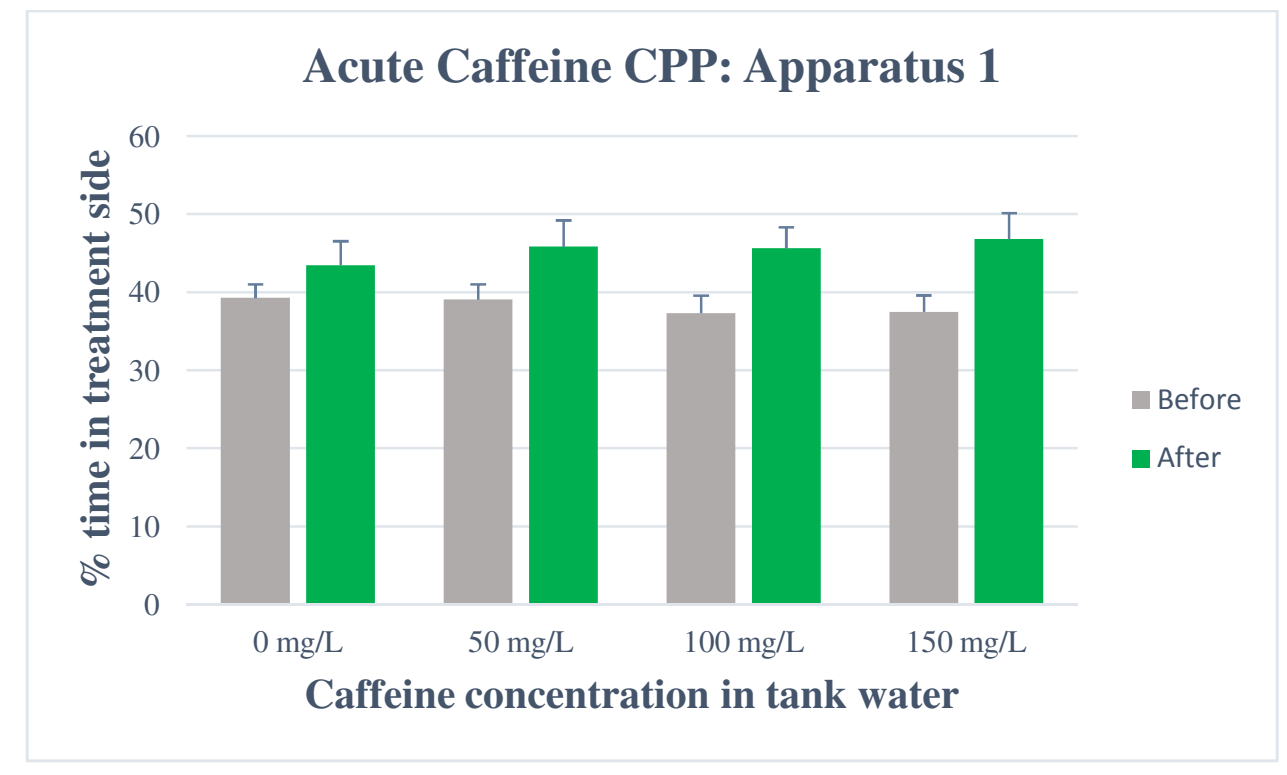

Figure 13. Acute caffeine apparatus $1 \mathrm{CPP}$ behavior: Paired samples analyses of time in treatment side before vs. after conditioning data expressed as mean $( \pm$ SEM $) .{ }^{*} p<0.0125$

Table 4

Acute Caffeine Apparatus 1: Paired Samples Analyses of Time in Treatment Side Before vs. After Conditioning

\begin{tabular}{|c|c|c|c|c|c|c|c|c|}
\hline \multirow[b]{2}{*}{ Dose } & \multicolumn{2}{|c|}{ Before } & \multicolumn{2}{|c|}{ After } & \multirow[b]{2}{*}{$n$} & \multirow[b]{2}{*}{$t$} & \multirow[b]{2}{*}{$p$} & \multirow[b]{2}{*}{$d$} \\
\hline & $M$ & $S D$ & $M$ & $S D$ & & & & \\
\hline $0 \mathrm{mg} / \mathrm{L}$ & 39.27 & 6.73 & 43.47 & 11.87 & 15 & 1.39 & 0.184 & 0.44 \\
\hline $50 \mathrm{mg} / \mathrm{L}$ & 39.06 & 7.99 & 45.82 & 13.96 & 17 & 1.63 & 0.122 & 0.59 \\
\hline $100 \mathrm{mg} / \mathrm{L}$ & 37.31 & 2.24 & 45.63 & 10.85 & 16 & 2.19 & 0.044 & 0.84 \\
\hline $150 \mathrm{mg} / \mathrm{L}$ & 37.47 & 2.13 & 46.80 & 12.93 & 15 & 2.52 & 0.024 & 0.86 \\
\hline
\end{tabular}

Note. Statistical significance was determined by a Bonferroni adjusted alpha level of $.05 / 4=0.0125$.

$* \mathrm{p}<.0125$

Planned contrasts revealed that the change in place preference towards the drugpaired side was not significantly different for either acute $50 \mathrm{mg} / \mathrm{L}, 100 \mathrm{mg} / \mathrm{L}$ or 150 
$\mathrm{mg} / \mathrm{L}$ of caffeine in apparatus 1 when compared to the change in place preference in control fish (Figure 14).

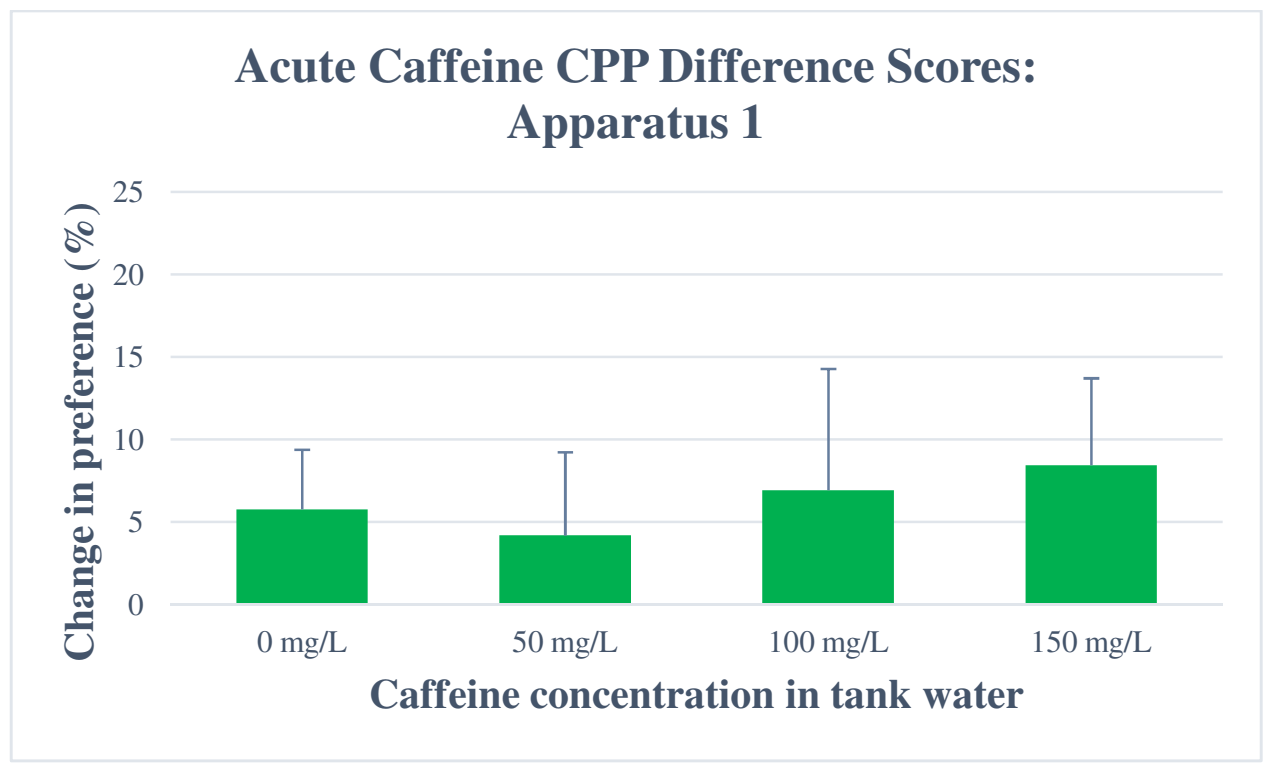

Figure 14. Acute caffeine apparatus 1 CPP difference scores: Change in place preference towards the drug-paired side. Data expressed as mean $( \pm \mathrm{SEM}) .{ }^{*} p<0.05 .{ }^{* *} p<0.01$

Results did not reveal a significant main effect of time (i.e., before conditioning vs. after conditioning) on dose of acute caffeine in apparatus $2, F(1,29)=3.98, \mathrm{p}=$ $0.055, \eta \mathrm{p}^{2}=0.12$. A significant interaction of time and caffeine was not revealed, $\mathrm{F}(3$, 29) $=0.043, p=0.99, \eta p^{2}=0.004$. Post-hoc paired samples $t$ tests did not reveal significant differences in place preference before and after conditioning for zebrafish treated acutely with either $0 \mathrm{mg} / \mathrm{L}, 50 \mathrm{mg} / \mathrm{L}, 100 \mathrm{mg} / \mathrm{L}$ or $150 \mathrm{mg} / \mathrm{L}$ of caffeine (see Figure 15 and Table 5). 


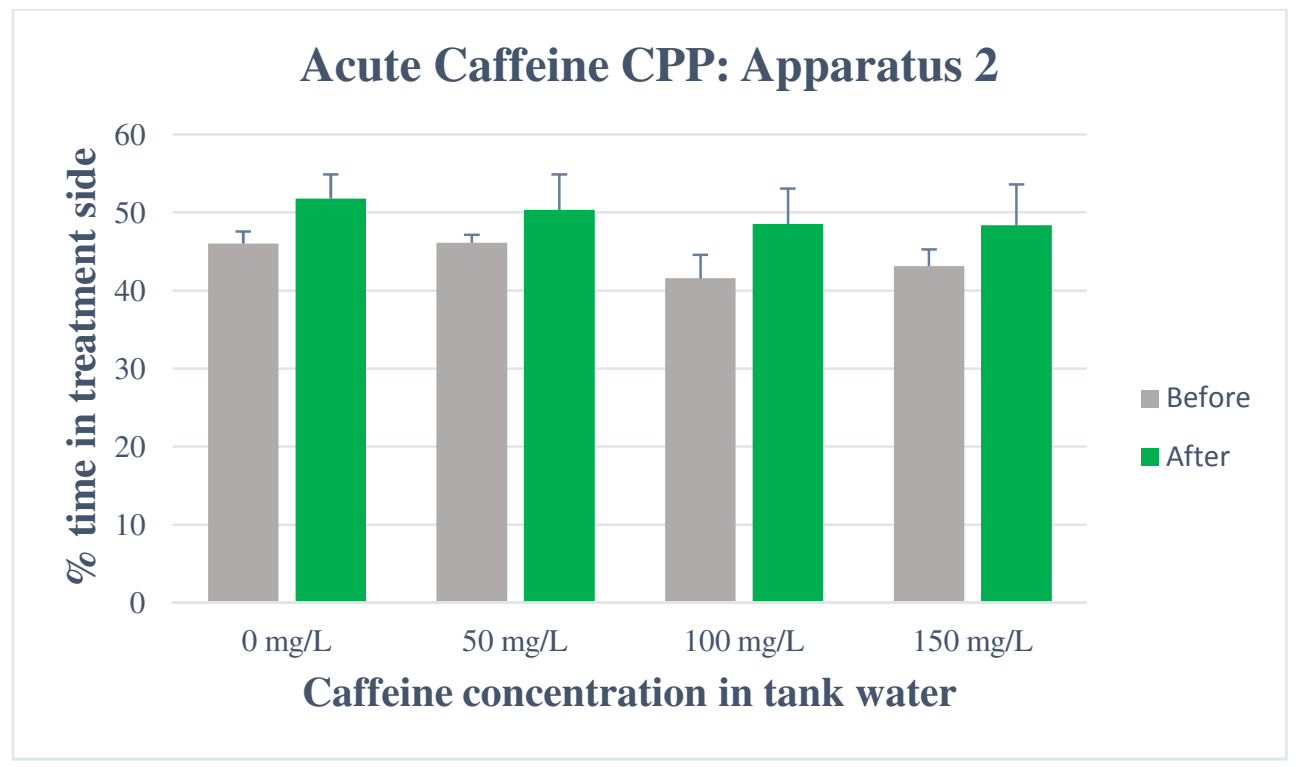

Figure 15. Acute caffeine apparatus $2 \mathrm{CPP}$ behavior: Paired samples analyses of time in treatment side before vs. after conditioning data expressed as mean $( \pm \mathrm{SEM}) .{ }^{*} p<0.0125$

Table 5

Acute Caffeine Apparatus 2: Paired Samples Analyses of Time in Treatment Side Before vs. After Conditioning

\begin{tabular}{|c|c|c|c|c|c|c|c|c|}
\hline \multirow[b]{2}{*}{ Dose } & \multicolumn{2}{|c|}{ Before } & \multicolumn{2}{|c|}{ After } & \multirow[b]{2}{*}{$n$} & \multirow[b]{2}{*}{$t$} & \multirow[b]{2}{*}{$p$} & \multirow[b]{2}{*}{$d$} \\
\hline & $M$ & $S D$ & $M$ & $S D$ & & & & \\
\hline $0 \mathrm{mg} / \mathrm{L}$ & 46.01 & 4.39 & 51.78 & 8.8 & 8 & 1.59 & 0.155 & 0.83 \\
\hline $50 \mathrm{mg} / \mathrm{L}$ & 46.12 & 3.13 & 50.31 & 13.72 & 9 & 0.89 & 0.430 & 0.42 \\
\hline $\begin{array}{c}100 \\
\mathrm{mg} / \mathrm{L}\end{array}$ & 41.58 & 8.49 & 48.50 & 12.92 & 8 & 0.94 & 0.378 & 0.63 \\
\hline $\begin{array}{r}150 \\
\mathrm{mg} / \mathrm{L}\end{array}$ & 43.13 & 6.12 & 48.36 & 14.88 & 8 & 0.94 & 0.379 & 0.46 \\
\hline
\end{tabular}

Note. Statistical significance was determined by a Bonferroni adjusted alpha level of $.05 / 4=0.0125$.

$* \mathrm{p}<.05 . * * \mathrm{p}<.0125$

Planned contrasts revealed that the change in place preference towards the drugpaired side was not significantly different for either acute $50 \mathrm{mg} / \mathrm{L}, 100 \mathrm{mg} / \mathrm{L}$ or 150 $\mathrm{mg} / \mathrm{L}$ of caffeine in apparatus 2 when compared to the change in place preference in control fish (Figure 16). 


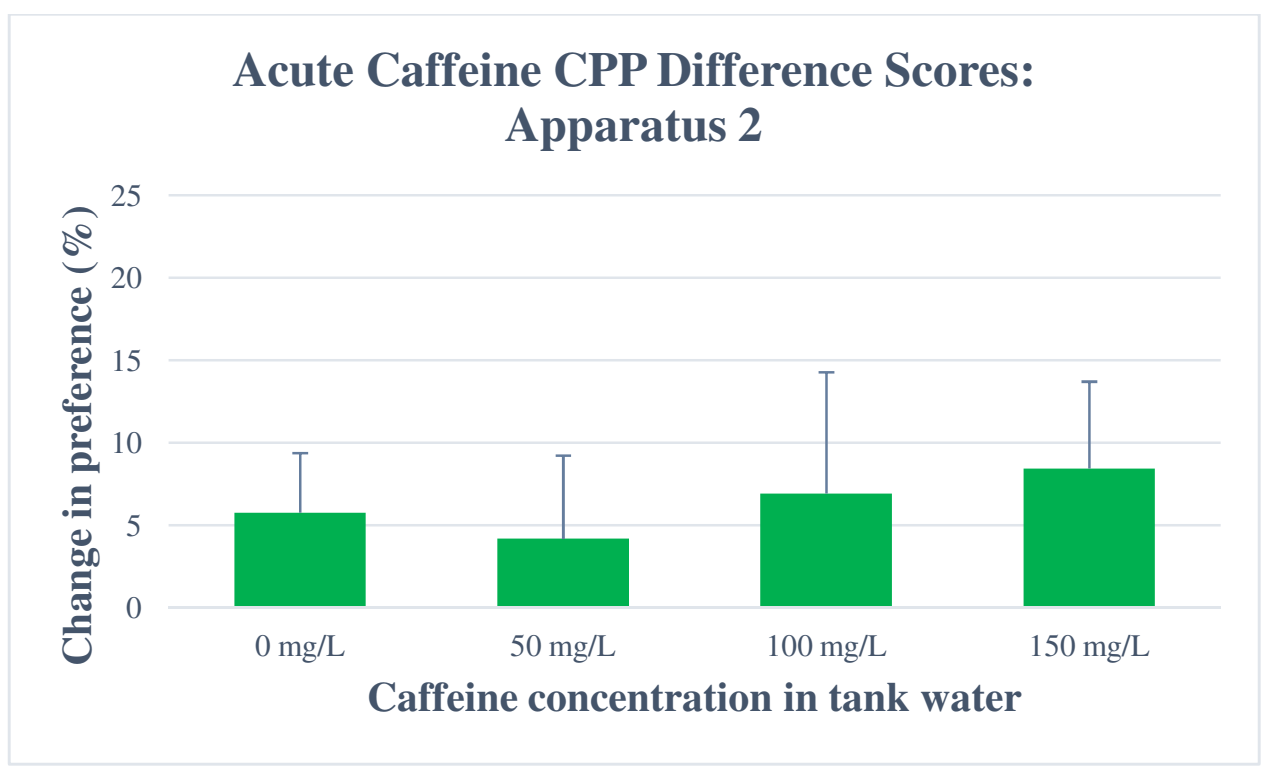

Figure 16. Acute caffeine apparatus $1 \mathrm{CPP}$ difference scores: Change in place preference towards the drug-paired side. Data expressed as mean $( \pm \mathrm{SEM}) .{ }^{*} p<0.05 . * * p<0.01$

Results revealed a significant main effect of time (i.e., before conditioning vs. after conditioning) on dose of acute caffeine in apparatus $2, \mathrm{~F}(1,27)=27.44$, p $<0.001$, $\eta p^{2}=0.50$. A significant interaction of time and caffeine was not revealed, $F(3,27)=$ 1.12, $\mathrm{p}=0.359, \eta \mathrm{p}^{2}=0.11$. Post-hoc paired samples $\mathrm{t}$ tests revealed a significant difference in place preference for zebrafish chronically treated with $50 \mathrm{mg} / \mathrm{L}$, indicating that these doses induced CPP behavior (see Figure 17 and Table 6). 


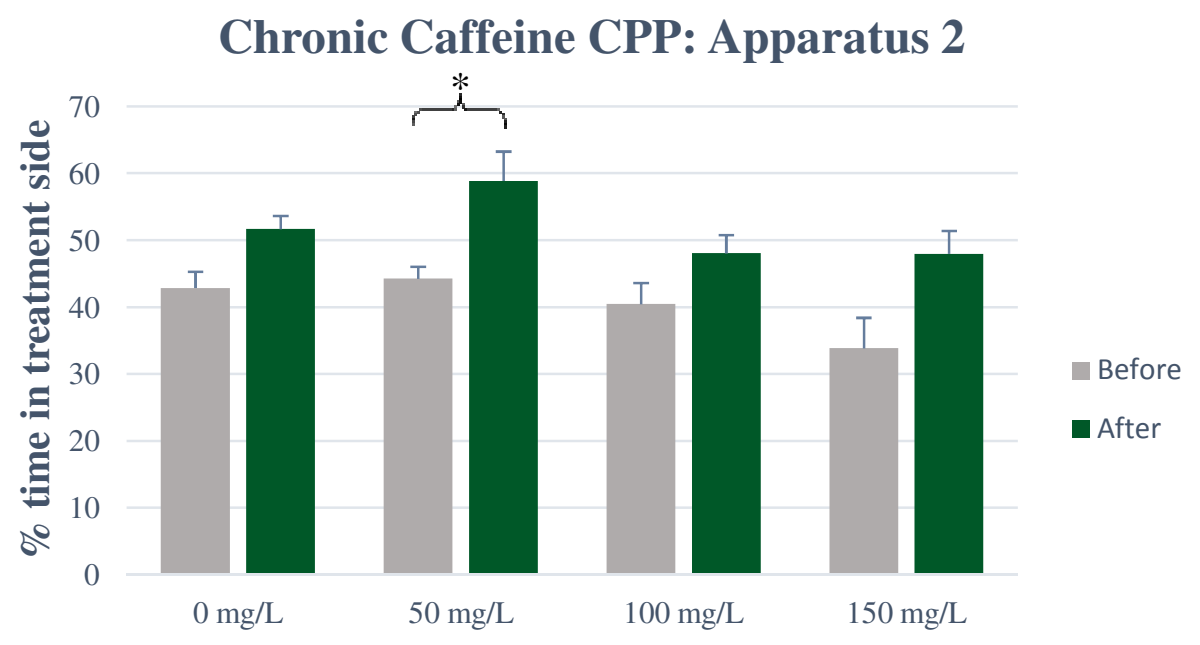

Caffeine concentration in tank water

Figure 17: Chronic caffeine apparatus 2 CPP behavior: Paired samples analyses of time in treatment side before vs. after conditioning. Data expressed as mean $( \pm \mathrm{SEM})$. ${ }^{*} p<$ 0.0125

Table 6

Chronic Caffeine Apparatus 2: Paired Samples Analyses of Time in Treatment Side Before vs. After Conditioning

\begin{tabular}{ccccccccc}
\hline & \multicolumn{2}{c}{ Before } & \multicolumn{2}{c}{ After } & & & & \\
\cline { 2 - 6 } & & & & & & & & \\
Dose & $M$ & $S D$ & $M$ & $S D$ & $n$ & $t$ & $p$ & $d$ \\
\hline $0 \mathrm{mg} / \mathrm{L}$ & 42.85 & 6.89 & 51.66 & 5.59 & 8 & 3.05 & 0.019 & 1.40 \\
$50 \mathrm{mg} / \mathrm{L}$ & 44.26 & 5.04 & 58.88 & 12.42 & 8 & 3.52 & $0.010^{*}$ & 1.54 \\
$100 \mathrm{mg} / \mathrm{L}$ & 40.46 & 8.87 & 48.05 & 7.7 & 8 & 2.56 & 0.038 & 0.91 \\
$150 \mathrm{mg} / \mathrm{L}$ & 33.86 & 12.06 & 47.97 & 9.05 & 7 & 2.24 & 0.066 & 1.32 \\
\hline
\end{tabular}

Note. Statistical significance was determined by a Bonferroni adjusted alpha level of $.05 / 4=0.0125$.

$* \mathrm{p}<.0125$

Planned contrasts revealed that the change in place preference towards the drugpaired side was not significantly different for chronic administration of either $50 \mathrm{mg} / \mathrm{L}$, $100 \mathrm{mg} / \mathrm{L}$ or $150 \mathrm{mg} / \mathrm{L}$ of caffeine in apparatus 2 when compared to the change in place preference in control fish (Figure 18). 


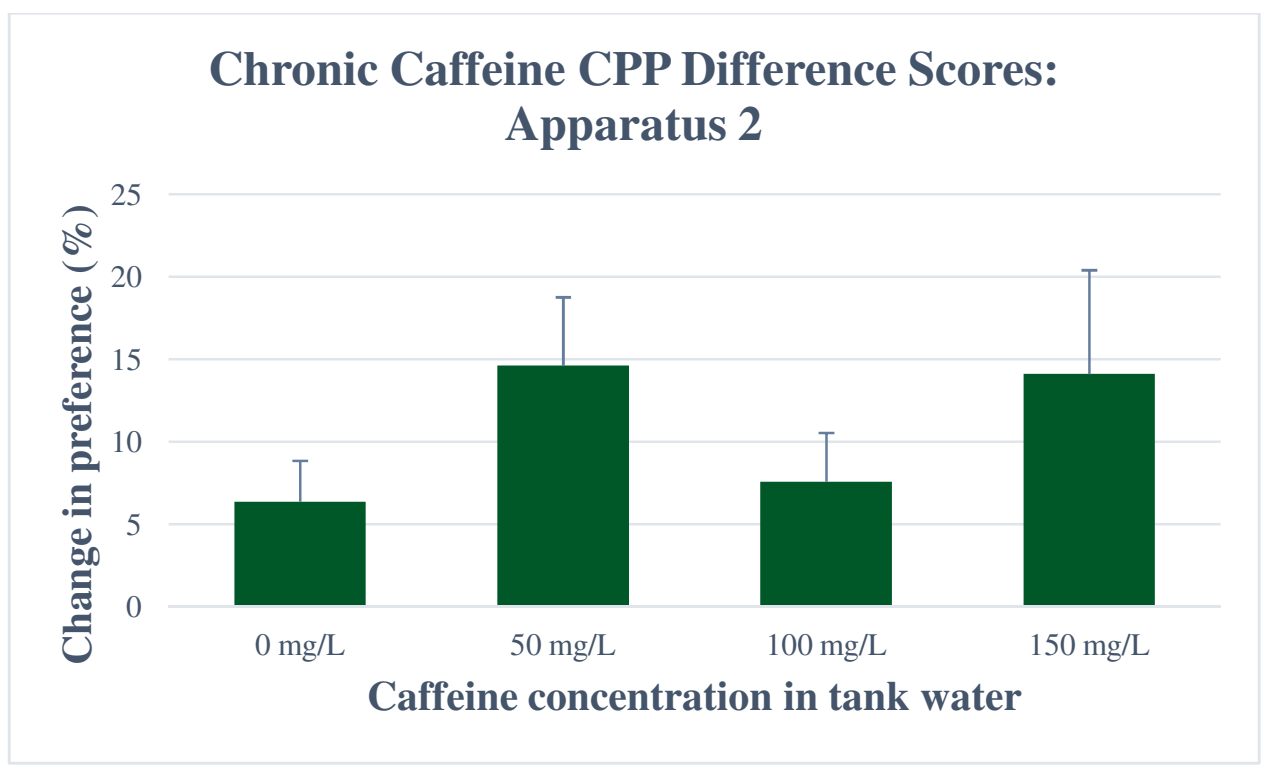

Figure 18. Chronic caffeine apparatus 2 difference scores: Change in place preference towards the drug-paired side. Data expressed as mean $( \pm \mathrm{SEM}) .{ }^{*} p<0.05 .{ }^{* *} p<0.01$

The effect of duration of treatment (i.e., acute vs. chronic) on change in place preference towards the caffeine paired side was not found to be significant, $F(1,56)=$ $2.189, p=0.145, \eta p^{2}=0.04$ (Figure 19). An analysis of simple effects showed that change in place preference towards the drug-paired side was not significantly different for any dose comparison across duration of treatment.

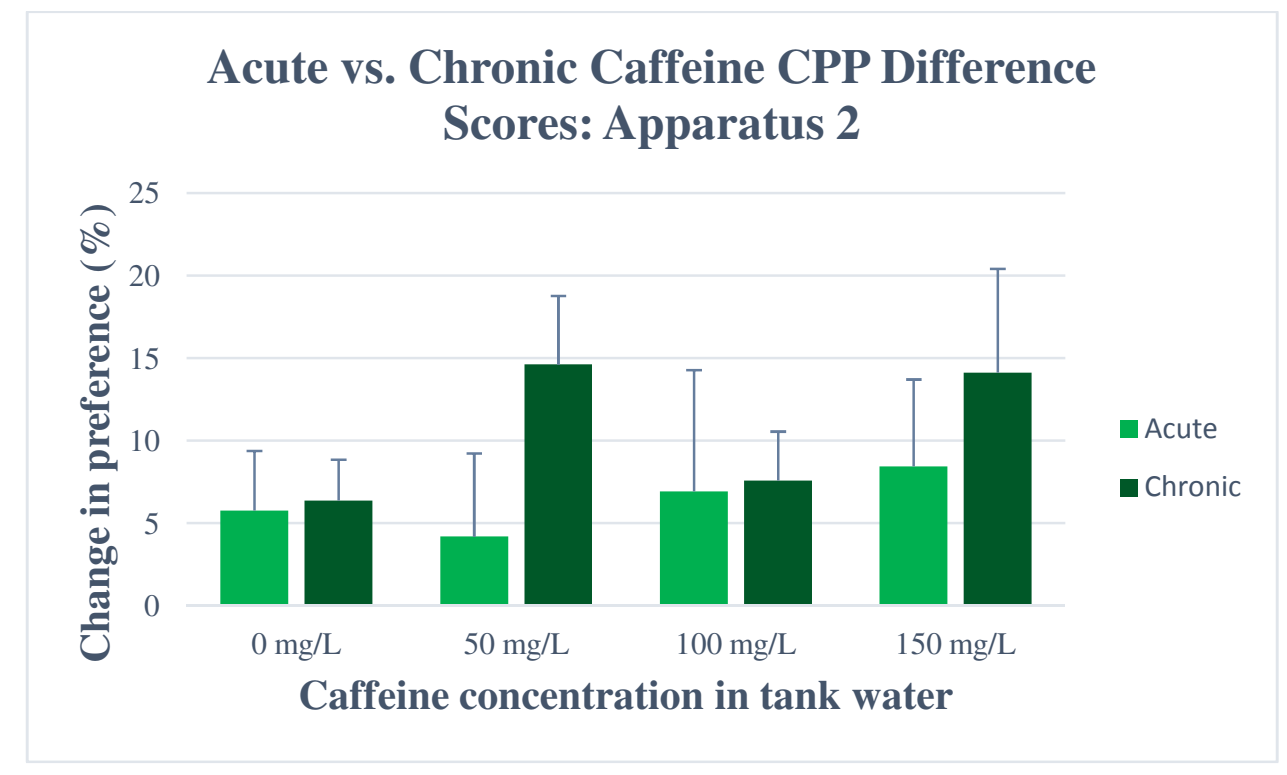


Figure 19. Acute vs. chronic caffeine CPP difference scores: Data expressed as mean $\left( \pm\right.$ SEM). ${ }^{*} p<0.05$. ${ }^{* *} p<0.01$

\section{Nicotine CPP Results}

Results revealed a significant main effect of time (i.e., before conditioning vs. after conditioning) on dose of acute nicotine in apparatus $2, \mathrm{~F}(1,26)=17.13, \mathrm{p}<0.001$, $\eta p^{2}=0.40$. A significant interaction of time and nicotine was not revealed, $F(3,27)=$ $\mathrm{F}(3,26)=1.43, \mathrm{p}=0.26, \eta \mathrm{p}^{2}=0.14$. Post-hoc paired samples t tests revealed a significant difference in place preference before and after conditioning for zebrafish treated acutely with $0 \mathrm{mg} / \mathrm{L}$ nicotine, but not for any other doses of nicotine (see Figure 20 and Table 7).

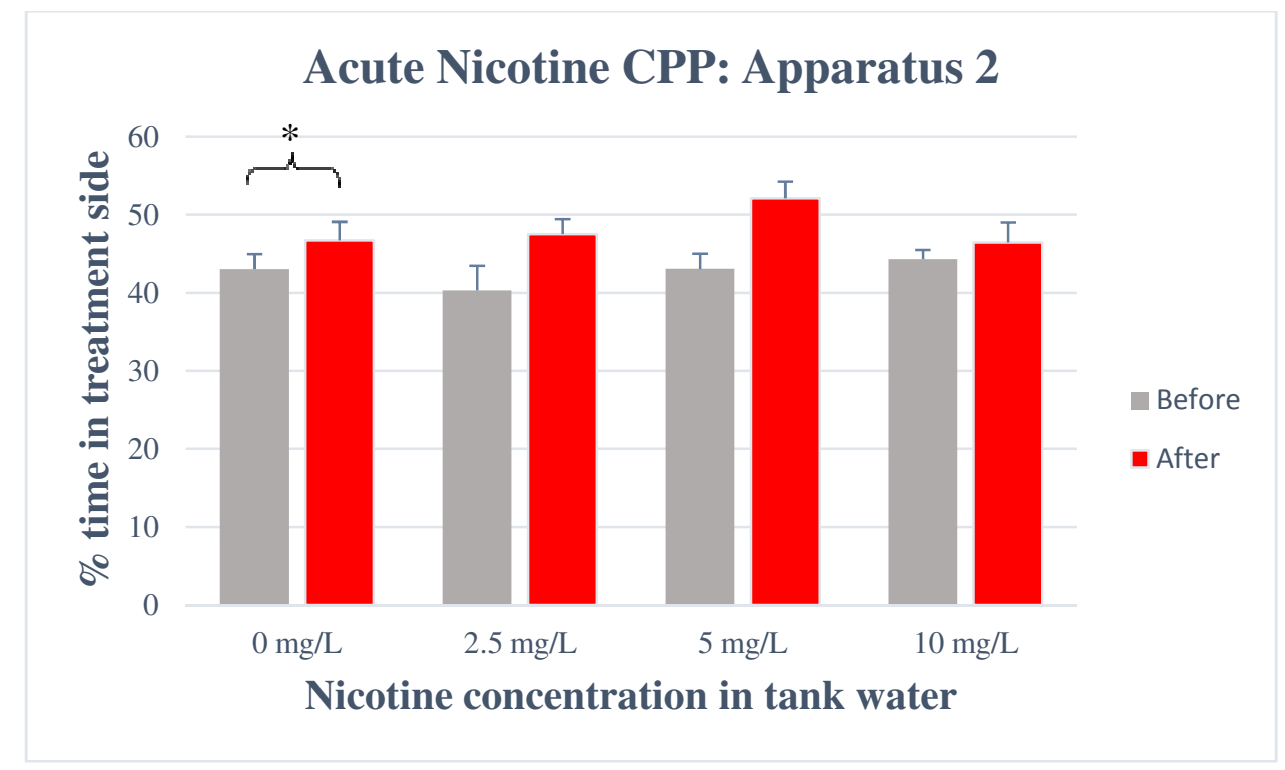

Figure 20. Acute nicotine apparatus 2 CPP behavior: Paired samples analyses of time in treatment side before vs. after conditioning data expressed as mean $( \pm \mathrm{SEM})$. $* p<0.0125$

Table 7

Acute Nicotine Apparatus 2: Paired Samples Analyses of Time in Treatment Side Before vs. After Conditioning 


\begin{tabular}{ccccccccc} 
& \multicolumn{2}{c}{ Before } & \multicolumn{2}{c}{ After } & & & & \\
\cline { 2 - 5 } Dose & $M$ & $S D$ & $M$ & $S D$ & $n$ & $t$ & $p$ & $d$ \\
\hline $0 \mathrm{mg} / \mathrm{L}$ & 43.03 & 5.09 & 46.70 & 6.33 & 7 & 3.78 & $0.009 *$ & 0.64 \\
$2.5 \mathrm{mg} / \mathrm{L}$ & 40.29 & 8.93 & 47.52 & 5.42 & 8 & 2.18 & 0.066 & 0.98 \\
$5 \mathrm{mg} / \mathrm{L}$ & 43.05 & 5.55 & 52.11 & 6.03 & 8 & 2.91 & 0.023 & 1.56 \\
$10 \mathrm{mg} / \mathrm{L}$ & 44.31 & 3.15 & 46.43 & 6.85 & 7 & 1.02 & 0.346 & 0.40 \\
\hline
\end{tabular}

Note. Statistical significance was determined by a Bonferroni adjusted alpha level of $.05 / 4=0.0125$.

$* \mathrm{p}<.0125$

Planned contrasts revealed that the change in place preference towards the drugpaired side was not significantly different for acute administration of either $2.5 \mathrm{mg} / \mathrm{L}, 5$ $\mathrm{mg} / \mathrm{L}$, or $10 \mathrm{mg} / \mathrm{L}$ of nicotine in apparatus 2 when compared to the change in place preference in control fish (Figure 21).

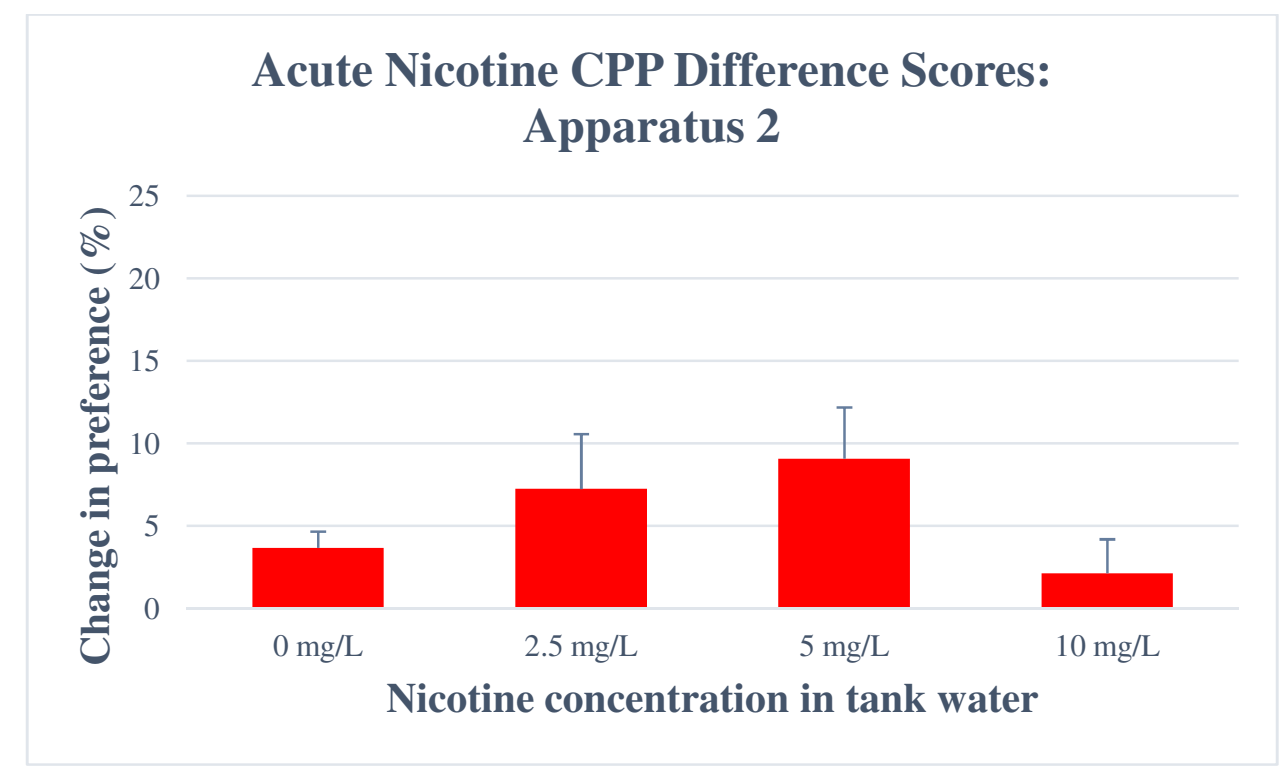

Figure 21. Acute ethanol apparatus 1 CPP difference scores: Change in place preference towards the drug-paired side. Data expressed as mean $( \pm \mathrm{SEM}) . * p<0.05 . * * p<0.01$

Results revealed a significant main effect of time (i.e., before conditioning vs. after conditioning) on dose of chronic nicotine in apparatus $2, \mathrm{~F}(1,24)=28.47$, p < $0.001, \eta p^{2}=0.54$. A significant interaction of time and nicotine was not revealed, $F(3$, 
24) $=1.22, p=0.324, \eta p^{2}=0.13$. Post-hoc paired samples t tests revealed no significant differences in place preference before and after conditioning for zebrafish treated chronically with $0 \mathrm{mg} / \mathrm{L}, 2.5 \mathrm{mg} / \mathrm{L}$ and $10 \mathrm{mg} / \mathrm{L}$ of nicotine (see Figure 22 and Table 8).

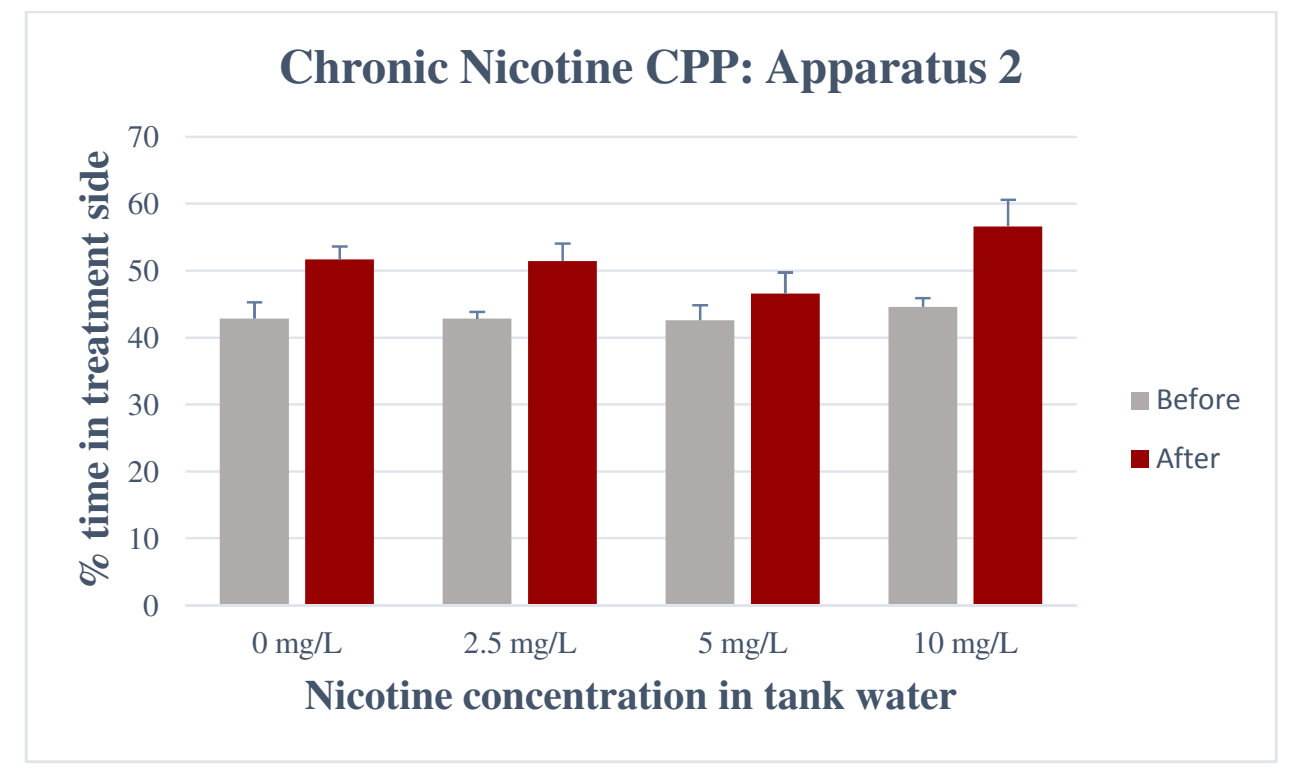

Figure 22. Chronic nicotine apparatus $2 \mathrm{CPP}$ behavior: Paired samples analyses of time in treatment side before vs. after conditioning data expressed as mean $( \pm \mathrm{SEM}) .{ }^{*} p<$ 0.0125

Table 8

Chronic Nicotine Apparatus 2: Paired Samples Analyses of Time in Treatment Side Before vs. After Conditioning

\begin{tabular}{ccccccccc}
\hline & \multicolumn{9}{c}{ Before } & \multicolumn{2}{c}{ After } & & & & \\
\cline { 2 - 5 } & & & & & & & \\
Dose & $M$ & $S D$ & $M$ & $S D$ & $n$ & $t$ & $p$ & $d$ \\
\hline $0 \mathrm{mg} / \mathrm{L}$ & 42.85 & 6.89 & 51.66 & 5.59 & 8 & 3.05 & 0.019 & 1.40 \\
$2.5 \mathrm{mg} / \mathrm{L}$ & 42.83 & 2.94 & 51.45 & 7.41 & 8 & 2.70 & 0.031 & 1.53 \\
$5 \mathrm{mg} / \mathrm{L}$ & 42.60 & 5.95 & 46.59 & 8.39 & 7 & 1.52 & 0.179 & 0.55 \\
$10 \mathrm{mg} / \mathrm{L}$ & 44.60 & 2.99 & 56.62 & 8.94 & 5 & 4.24 & 0.013 & 1.80 \\
\hline
\end{tabular}

Note. Statistical significance was determined by a Bonferroni adjusted alpha level of $.05 / 4=0.0125$.

$* \mathrm{p}<.0125$ 
Planned contrasts revealed that the change in place preference towards the drugpaired side was not significantly different for chronic administration of either $2.5 \mathrm{mg} / \mathrm{L}, 5$ $\mathrm{mg} / \mathrm{L}$, or $10 \mathrm{mg} / \mathrm{L}$ of nicotine in apparatus 2 when compared to the change in place preference in control fish (Figure 23).

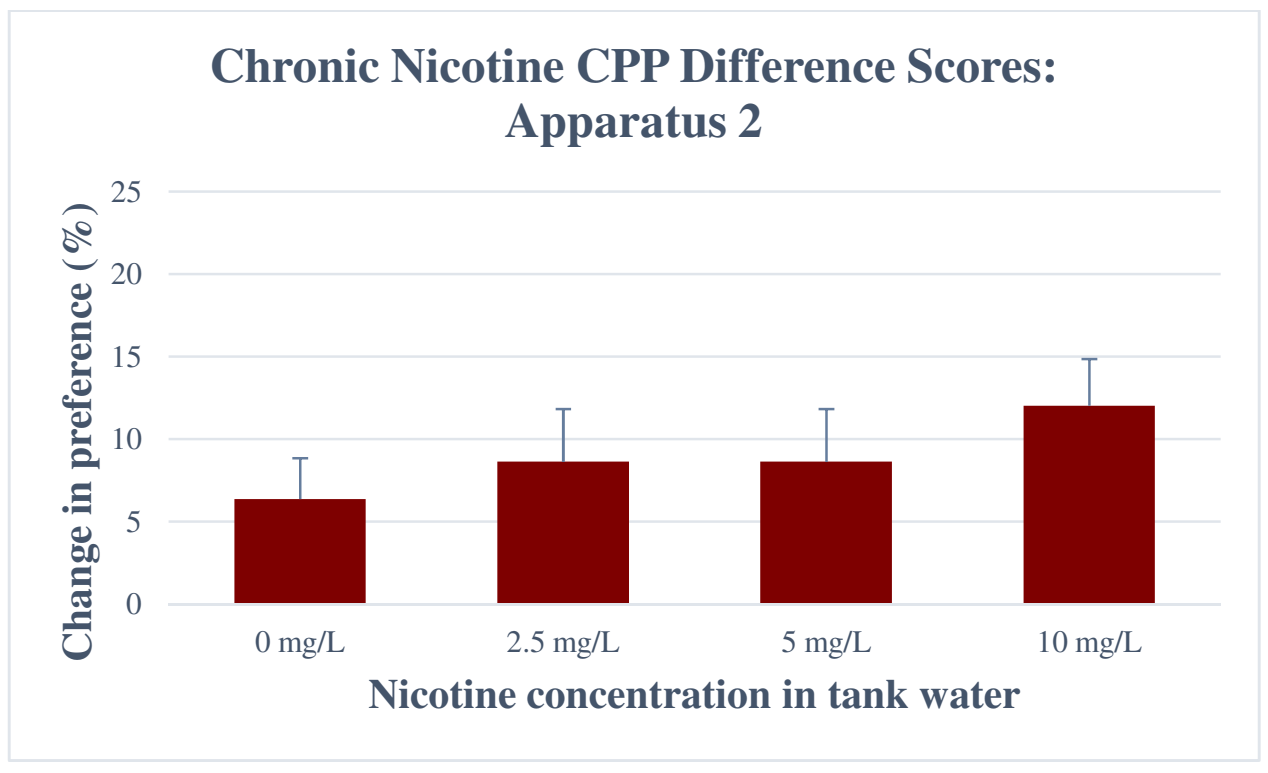

Figure 23. Chronic nicotine apparatus 2 CPP difference scores: Change in place preference towards the drug-paired side. Data expressed as mean $( \pm \mathrm{SEM}) .{ }^{*} p<0.05$. $* * p<0.01$

The effect of duration of treatment (i.e., acute vs. chronic) on change in place preference towards the nicotine-paired side was not found to be significant, $F(1,50)=$ 1.282, $p=0.263, \eta p^{2}=0.03$ (Figure 24). An analysis of simple effects showed that change in place preference towards the drug-paired side was significantly greater for zebrafish treated chronically with $10 \mathrm{mg} / \mathrm{L}$ of nicotine compared to zebrafish treated acutely with $10 \mathrm{mg} / \mathrm{L}$ of nicotine $F(1,50)=5.205, p=0.027, \eta \mathrm{p}^{2}=0.094$. 


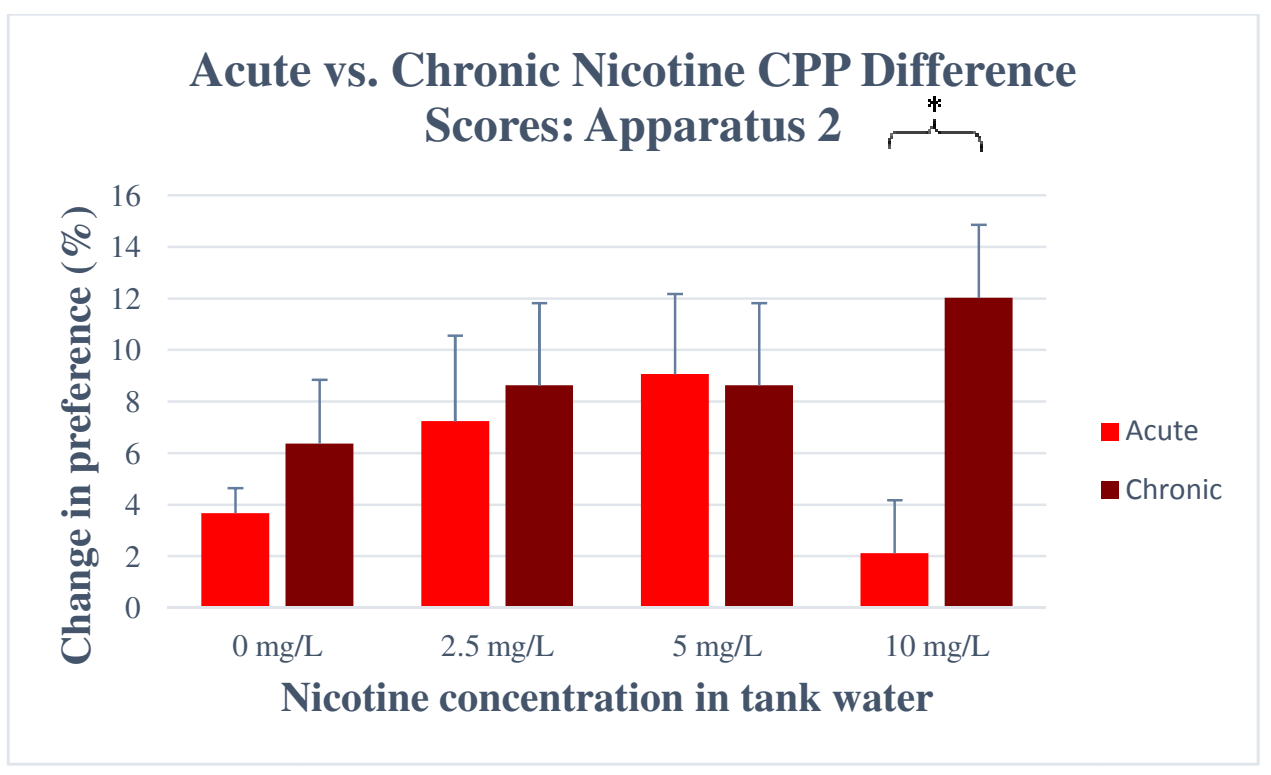

Figure 24. Acute vs. chronic nicotine CPP difference scores: Data expressed as mean $\left( \pm\right.$ SEM). ${ }^{*} p<0.05 . * * p<0.01$

Side Preference and Conditioning Order Results

To evaluate potential side bias, the relationship between each distinct environment of both apparatus designs and baseline side preferences were evaluated. Baseline side preference was determined by spending between $50.1 \%$ and $79.9 \%$ of time in one environment during the 10 minute baseline testing period. In apparatus design 1 , more fish displayed a baseline preference towards the white side $(n=75)$ than the dotted side $(n=48)$. A binomial test indicated that the proportion of zebrafish who preferred the white side of $61 \%$ was significantly higher than the hypothesized proportion of $50 \%, p=$ 0.019 (Figure 25) 


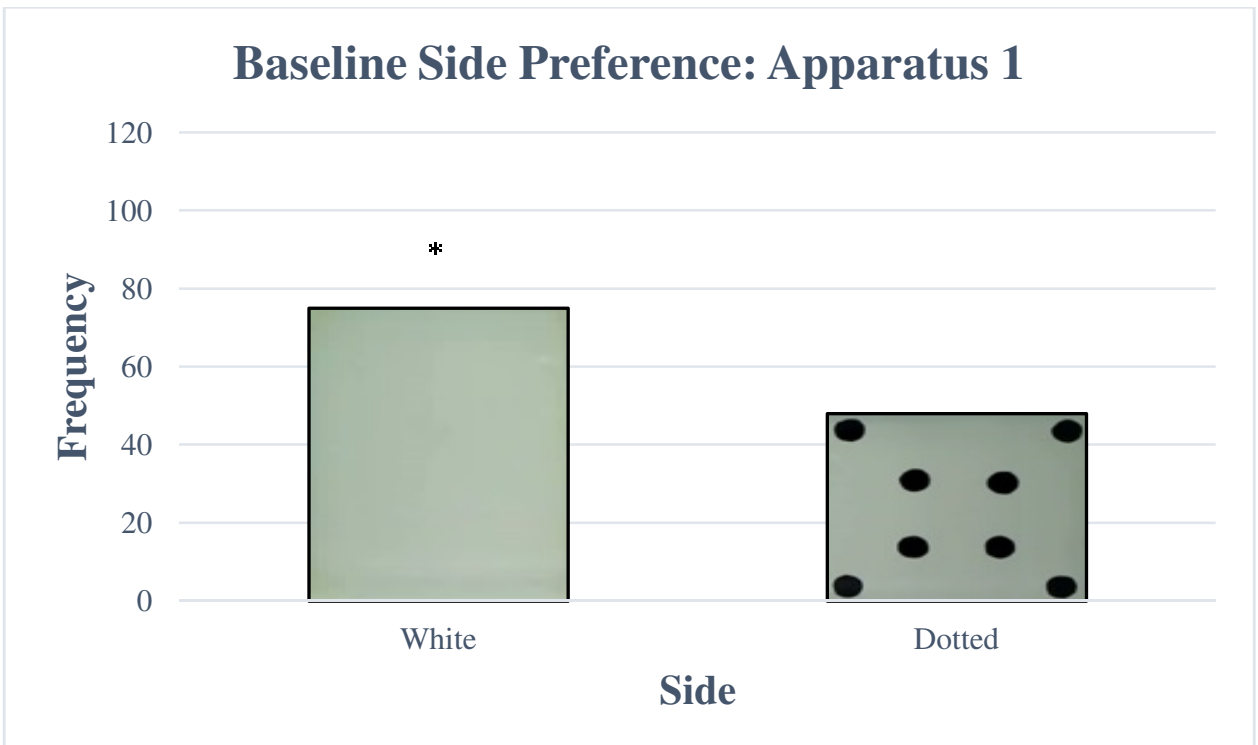

Figure 25. Initial time spent in preferred sides of the CPP apparatus 1 during baseline testing. Data expressed as mean $( \pm \mathrm{SEM}) .{ }^{*} p<0.05 .{ }^{* *} p<0.01$

An independent samples t-test that revealed there was no significant effect of the side zebrafish preferred during baseline testing on the time spent in that side $(p=0.318)$. To summarize this result, animals that initially preferred the white side spent roughly the same amount of time in that side during baseline preference testing as did fish that initially preferred the dotted side (Figure 26).

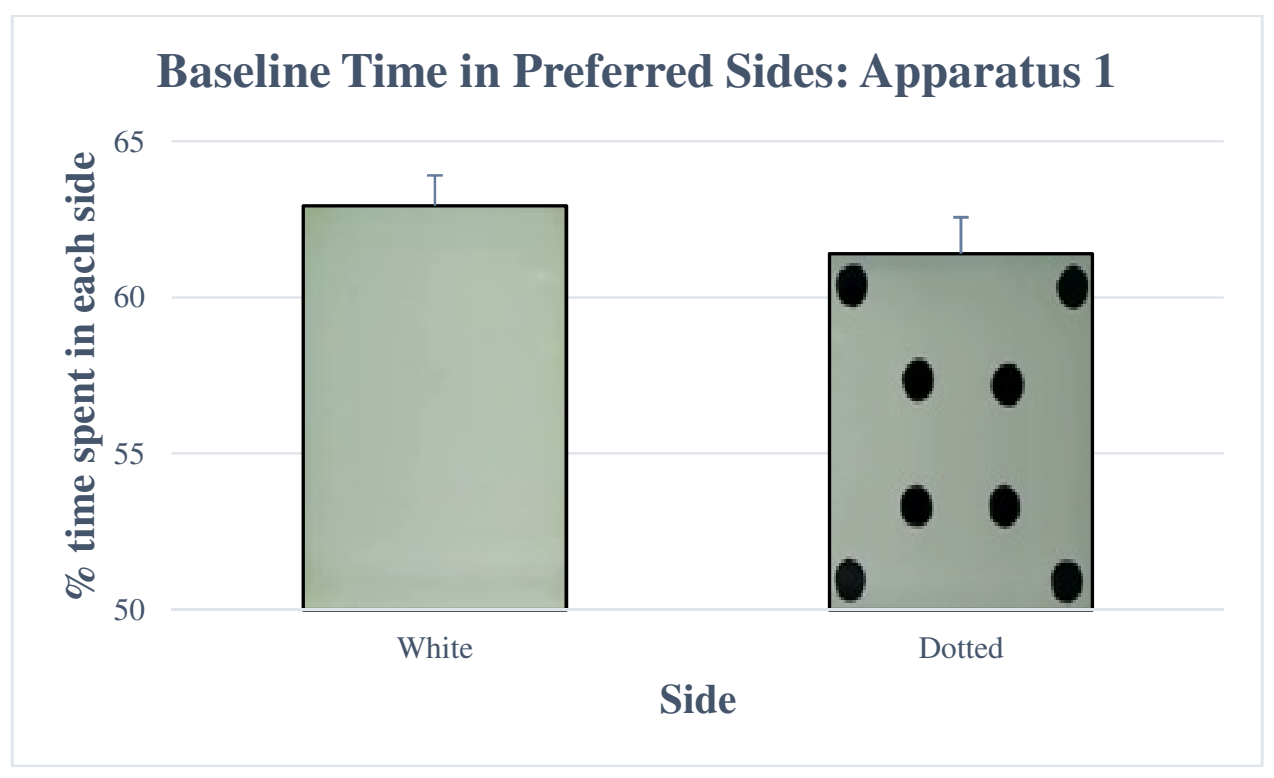


Figure 26. Initial time spent in preferred sides of the CPP apparatus 1 during baseline testing. Data expressed as mean $( \pm$ SEM). $* p<0.05$. $* * p<0.01$

In apparatus design 2, more fish displayed a baseline preference towards the dotted side $(n=110)$ than the rectangle side $(n=83)$. A binomial test indicated that the proportion of zebrafish who preferred the dotted side of $57 \%$ was not significantly higher than the hypothesized proportion of $50 \%, p=0.061$ (Figure 27).

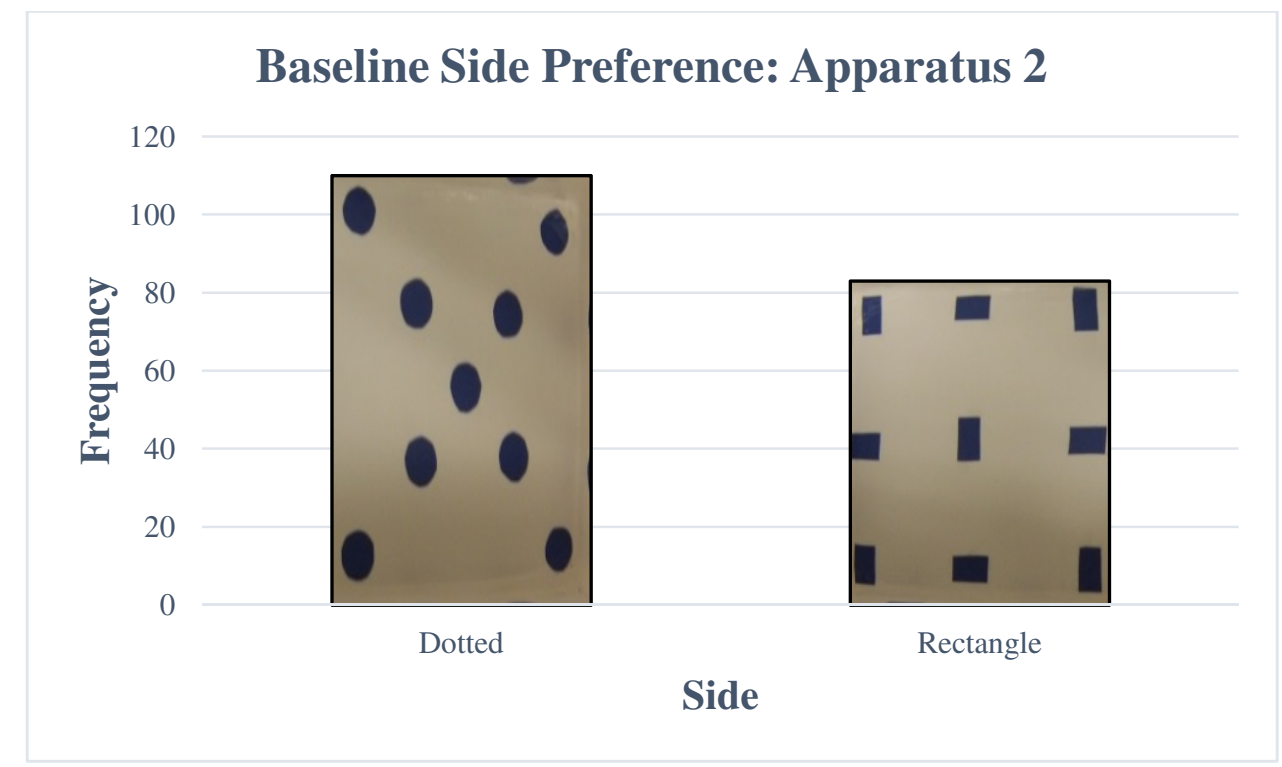

Figure 27. Initial time in preferred sides of the CPP apparatus 2. Data expressed as mean $\left( \pm\right.$ SEM). ${ }^{*} p<0.05 . * * p<0.01$

An independent samples t-test revealed that there was no significant effect of the side zebrafish preferred during baseline testing on the time spent in that side in apparatus design $2(\mathrm{p}=0.617)$. Animals that initially preferred the dotted side during baseline testing spent about the same amount of time in that side, as did animals that initially preferred the rectangle side (Figure 28) 


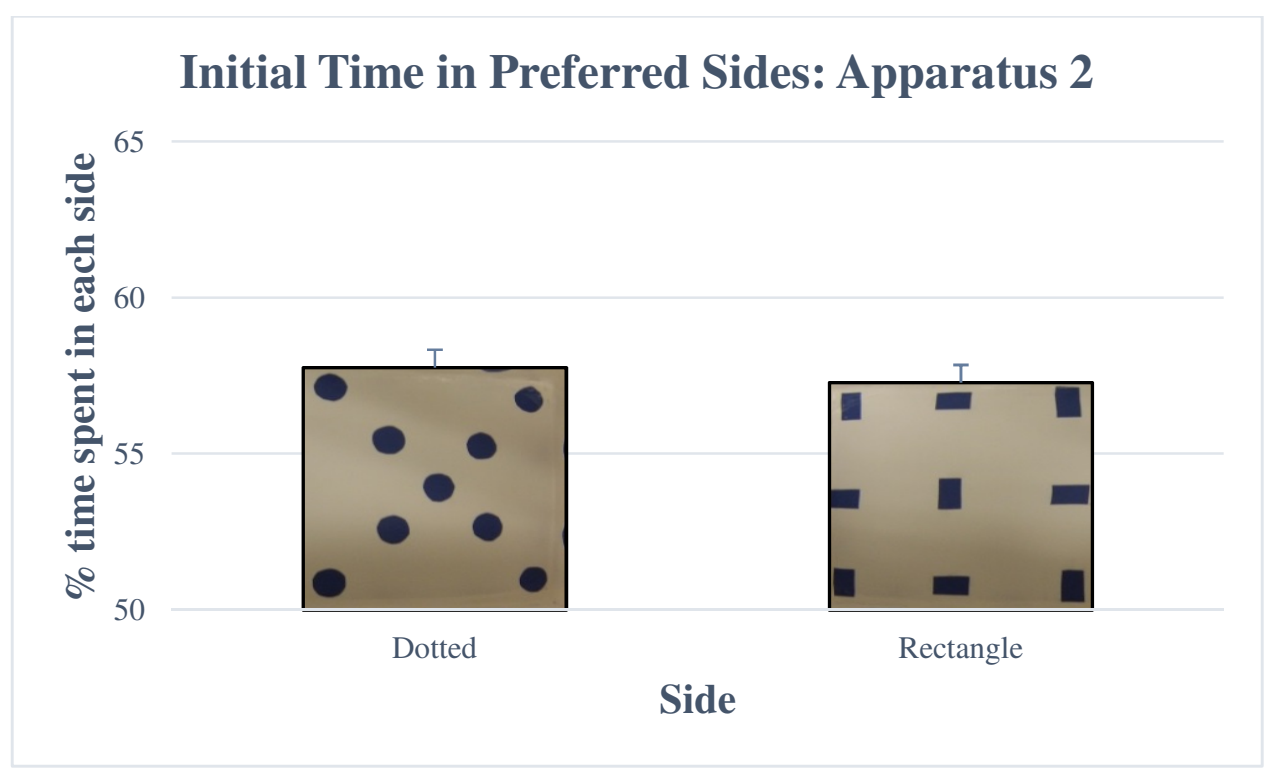

Figure 28. Initial time in preferred sides of the CPP apparatus 2. Data expressed as mean $( \pm$ SEM). $* p<0.05 . * * p<0.01$

The effect of conditioning order on change in place preference towards the drug paired side was also assessed (Figure 29). Zebrafish that were first placed in their preferred side and administered only water, and then placed in their non-preferred side and administered drug, displayed a significantly greater change in place preference towards the drug paired side than fish that were first placed in their non-preferred side and received drug, and then placed in their preferred side and received water, $t(191)=$ $3.21, p=0.002, d=0.46$ (Figure 29). To summarize this effect, zebrafish that received drug second during conditioning displayed a greater change in place preference than zebrafish that received drug first. 


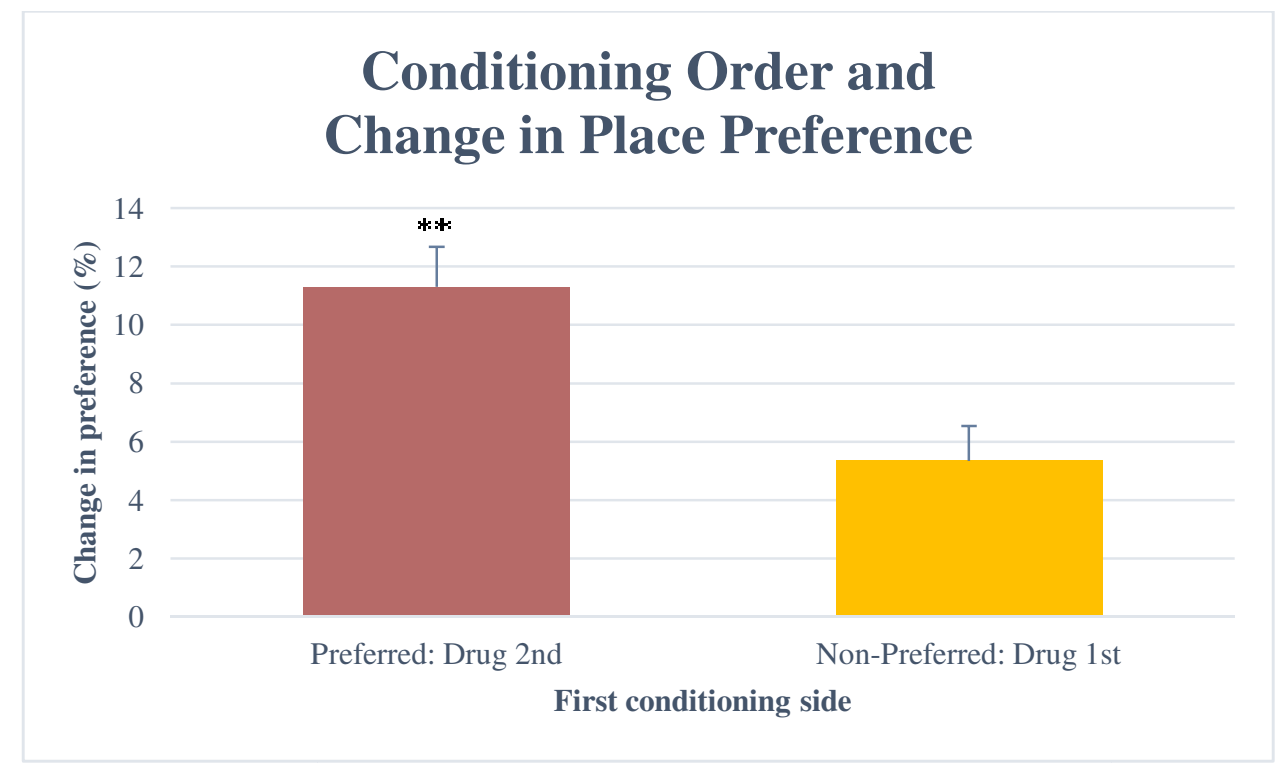

Figure 29. The effect of conditioning order on change in place preference behavior. Data expressed as mean $( \pm \mathrm{SEM}) .{ }^{*} p<0.05 . * * p<0.01$ 


\section{CHAPTER IV}

\section{DISCUSSION}

The rewarding effects of ethanol, caffeine and nicotine were assessed in this study by evaluating the ability of these substances to increase place preference for an environment that was not initially preferred by zebrafish. Behavioral paradigms historically tested in rodents, such as CPP, have only recently been applied in zebrafish neurobehavioral research (Darland \& Dowling, 2001). Therefore, concerted efforts at replication of previously reported findings, in addition to assessing novel and untested compounds and doses, will help establish good face validity of the zebrafish CPP model. The advantageous characteristics of the zebrafish, when coupled with a relatively simple CPP procedure that can be carried out in a short period of time with multiple animals being tested simultaneously, establish this model as a reliable and effective model of drug reward. Although the zebrafish brain and behavior are not homologous to that of mammals, anatomical organization and the biology of the nervous system are generally conserved among vertebrates, mediating many of the same behaviors.

These results demonstrate that ethanol is capable of inducing CPP in adult zebrafish following a single (i.e., acute) 20 minute administration with concentrations of $0.25 \%, 0.50 \%$ and $1.00 \% \mathrm{v} / \mathrm{v}$ in apparatus design 1 , similar to previously published findings (Kily et al., 2008; Parmar et al., 2011), with the exception of the $0.50 \%$ concentration, which is being reported for here the first time. However, these doses of acute ethanol were not found to induce CPP behavior in zebrafish in apparatus design 2 . Results also indicated that change in place preference towards the ethanol-paired side was significantly greater following acute $0.50 \%$ and $1.00 \%$ ethanol compared to control in 
apparatus design 1, but not following acute $0.25 \%$. In apparatus design 2 , change in place preference towards the ethanol-paired side was greater following acute $0.25 \%$ ethanol, but not $0.50 \%$ or $1.00 \%$ ethanol when compared to control. These differential results following acute ethanol treatment between apparatus designs may be attributable to overall lower sample sizes in apparatus 2. It is also possible that these differences are due to the nature of the environmental stimuli used in each apparatus design. For example, zebrafish may have been better able to differentiate between the two environments in apparatus 1 (i.e., white vs. black dots) than in apparatus 2 (i.e., blue dots vs. blue rectangles). Although a baseline bias for the white environment was found in apparatus design 1, the high degree of visual distinction between environments may be necessary for animals to develop a conditioned association. Future investigation into the ability of zebrafish to differentiate between various environmental stimuli and the testing of other apparatus designs is warranted.

Ethanol has been reported to produce a linear-like relationship of dose-dependent increases in dopamine production following a 1 hour exposure to the same concentrations tested in the present experiment, $0.00 \%, 0.25 \%, 0.50 \%$, and $1.00 \% \mathrm{v} / \mathrm{v}$ ethanol (Chatterjee \& Gerlai, 2009). Although direct experimental evidence is needed, it appears that dopamine may play a role in the ability of acute alcohol to produce CPP in zebrafish. In a previous study from our laboratory, blood alcohol concentrations (BAC) were measured in zebrafish following a 10 minute immersion in $0.125 \%, 0.25 \%, 0.50 \%$ and $1.00 \% \mathrm{v} / \mathrm{v}$ ethanol. The first three doses produced a relatively linear increase in blood alcohol levels $(0.050 \%, 0.058 \%$, and $0.065 \%$ respectively), and $1.00 \%$ resulted in BAC of $\sim 0.10 \%$, verifying the absorption of ethanol through immersion in a bath solution 
(Echevarria et al., 2010). Thus, the linear-like changes in place preference that were observed following acute ethanol exposure in apparatus 1 are more closely related to the aforementioned biological data than acute ethanol results from apparatus 2 , providing further support that the distinctive nature of apparatus design 1 may more appropriate for CPP testing in zebrafish.

Additionally, the effects of chronic administration of ethanol on place preference behavior was investigated in the present study and it was found that no dose of ethanol significantly increased time spent in the non-preferred environment. Moreover, no changes in place preference between-groups were found to be significant. Compared to acute ethanol treatment, change in place preference following chronic $0.25 \%$ ethanol was found to be significantly less than acute $0.25 \%$ ethanol, but no other differences in this regard were revealed. There was a relatively high amount of variation between zebrafish chronically treated with $0.25 \%$ ethanol (i.e., $\mathrm{SD}=18.36$ ), especially when compared to zebrafish acutely treated with $0.25 \%$ ethanol (i.e., $\mathrm{SD}=7.86$ ). A larger sample size may have mitigated this high degree of variation in zebrafish chronically treated with $0.25 \%$ ethanol, potentially influencing the aforementioned difference.

Furthermore, the results from chronic ethanol exposure in this study do not conform well to previously reported findings in the zebrafish literature. For example, it has been reported that both acute and chronic administration of $0.25 \%$ and $1.00 \%$ ethanol in a CPP task induced significant place preference behavior for the ethanol-paired environment (Chacon \& Luchiari, 2014). It has also been found that one week of conditioning with $1.00 \%$ ethanol significantly increased place preference in comparison to 1 day (i.e., acute) and 3 weeks of conditioning with $1.00 \%$ ethanol significantly 
increased place preference relative to both 1 day and 1 week of conditioning (Parmar et al., 2011). Due to such findings, it is unlikely that the overall lack of a CPP response in zebrafish chronically treated with ethanol is the result of pharmacologically impaired learning. Similar to acute ethanol findings in apparatus 2, the overall lack of observed effect in the present study may be attributable to reduced environmental distinction and overall low sample sizes (i.e., $n=6-8$ ).

The behavioral and pharmacological responses of animals to alcohol (i.e., ethanol), such as sensitivity, tolerance, and dependence, is known to be influenced by the genetic make-up of the organism (Crabbe, Belknap, \& Buck, 1994). In zebrafish, genetic-strain dependent behavioral differences in startle responses, social interactions, and tolerance have been observed following chronic ethanol exposure, albeit brain alcohol levels were comparable among strains (Dlugos \& Rabin, 2003). The fact that the responses of zebrafish to chronic ethanol in the present study do not reflect those that have been reported by other researchers may be due to differential genetic compositions of zebrafish. In this regard, future investigation into the involvement of genotype in regulating the rewarding effects of ethanol is warranted.

In addition to ethanol, CPP behavior in zebrafish following acute and chronic caffeine administration was investigated. In the present study, in both apparatus designs, acute caffeine was not found to significantly increase time spent in the drug-paired side, and no statistically significant differences were revealed from between-subjects comparisons of difference scores. In rodents, a single (i.e., acute) intraperitoneal injection of caffeine at $0.8 \mathrm{mg} / \mathrm{kg}, 3 \mathrm{mg} / \mathrm{kg}$, and $6 \mathrm{mg} / \mathrm{kg}$ did not induce CPP, although $1.5 \mathrm{mg} / \mathrm{kg}$ administration did produce CPP (Patkina \& Zvartau, 1998). Thus, evaluating rewarding 
effects of acute caffeine in zebrafish will require further investigation and a broader range of doses to be evaluated. To date, there are no reports that caffeine can cross the bloodbrain barrier in zebrafish. However, caffeine is observed to alter behavioral and endocrine phenotypes and is thus inferred that it has entered the brain and systemic circulation. Another possible explanation for the overall, non-rewarding effects of acute caffeine that have been reported in the present study is that acute caffeine may increase anxiety-like behaviors in zebrafish (El Yacoubi, Ledent, Parmentier, Costentin, \& Vaugeois, 2000; Sawyer, Julia, \& Turin, 1982).

Behavioral paradigms, such as the novel tank test, act as models of zebrafish anxiety and capitalize on innate behavioral responses of zebrafish to primarily dive and spend time on the bottom the novel tank (geotaxis). Immersion in $100 \mathrm{mg} / \mathrm{L}$ caffeine for 15-mins reduced transitions to top and time spent in upper portions of the novel tank, and increased instances of erratic movements. (Egan et al. 2009). Additionally, immersion in $250 \mathrm{mg} / \mathrm{L}$ caffeine for 20-mins significantly increased circulating cortisol levels, increased latency to upper half, freezing bouts, freezing duration, and decreased average velocity and distance traveled in the novel tank, all of which are indicative phenotypes of anxiety in zebrafish. (Cachat et al., 2010; Wong et al., 2010). Albeit CPP is not a measure of anxiety, any anxiety-like effects that are induced by acute caffeine could potentially interfere with the sensitivity of CPP to measure reward. In future studies, it would be beneficial to investigate if acute caffeine does indeed increase anxiety-like behaviors in zebrafish at the doses tested in the present study.

Clinical data supports the link between caffeine consumption and the development of dependence (i.e., addiction) (Anderson \& Juliano, 2012; Juliano \& 
Griffiths, 2004; Striley, Griffiths, \& Cottler, 2011). In humans, caffeine is typically consumed over long (e.g., chronic) periods of time. When zebrafish were administered caffeine chronically a significant increase in place preference was found following treatment with $50 \mathrm{mg} / \mathrm{L}$, but not at higher, potentially anxiogenic doses. The primary mechanism of caffeine in the brain is non-selective antagonism of adenosine receptors, the main targets being $\mathrm{A}_{1}$ and $\mathrm{A}_{2 \mathrm{~A}}$ adenosine receptor subtypes (Fredholm, Bättig, Holmén, Nehlig, \& Zvartau, 1999; Nehlig, 1999). The adenosinergic system of cyprinid fish is similar to that of mammals (Maximino et al., 2011), and zebrafish have been found to express $A_{1}, A_{2 A 1}, A_{2 A 2}$ and $A_{2 B}$ receptor subtypes 24 hours post-fertilization, the mRNA expression of which has been found to be modulated by caffeine exposure (Capiotti et al., 2011b). Adenosine is known to be a neuromodulator of dopamine transmission in the CNS (Cauli \& Morelli, 2005). Specifically, stimulation of adenosine A2 receptors by adenosine agonists has been found to decrease affinity of dopamine D2 receptors for dopamine in humans and rodents (Ferre, von Euler, Johansson, Fredholm, \& Fuxe, 1991). Conversely, the antagonistic action of caffeine at A2 receptors inhibits the negative modulatory effects of adenosine on dopamine, and results in a potentiation of dopaminergic neurotransmission (Ferré, Fuxe, von Euler, Johansson, \& Fredholm, 1992; Garrett \& Griffiths, 1997; Nehlig, 1999; Pollack \& Fink, 1995). The dopaminergic system is highly conserved in zebrafish, the activation of which may underlie the rewarding effects of caffeine (Rink \& Wullimann, 2002a, 2002b). Collectively, this suggests that the rewarding effects of chronic caffeine treatment may be mediated by long-term antagonism of adenosine receptors and the association indirect dopamine transmission. 
Lastly, the rewarding effects of acute and chronic nicotine were evaluated in this study, albeit only in apparatus design 2. In the acute cohort, zebrafish in the control group displayed a significant increase in time spent in their non-preferred side, an effect that was not observed in zebrafish after receiving any dose of nicotine, and no betweengroup differences in change in preference were found. The significant change in the acute control group was likely due to chance, as evidenced by a small change in place preference (i.e., a 3\% increase). Zebrafish chronically administered nicotine did not display an increase in time spent in their non-preferred side following conditioning and there were no differences between-groups. Zebrafish that were chronically administered $10 \mathrm{mg} / \mathrm{L}$ nicotine spent significantly more time in the nicotine-paired side than zebrafish who received acute $10 \mathrm{mg} / \mathrm{L}$ of nicotine.

In the literature, nicotine has been reported to induce CPP behavior in zebrafish at several doses and durations of exposure. A single administration (i.e., acute) of 3, 30, 60, and $300 \mu \mathrm{mol} 1^{-1}$ were all found to significantly increase place preference towards the drug-paired environment after conditioning (Kily et al., 2008). The greatest effect was observed in animals who received $30 \mu \mathrm{mol} 1^{-1}$ of nicotine, which spent $70 \%$ more time in the nicotine paired environment after conditioning in relation to before conditioning. Zebrafish were also found to demonstrate CPP following 21 days of abstinence after receiving $30 \mu \mathrm{mol}^{-1}$ of nicotine. Three conditioning sessions with a $300 \mu \mathrm{mol}^{-1}$ of nicotine significantly decreased place preference, suggesting conditioned place aversion (CPA). In a separate study a biased apparatus was employed, in that 20 minute exposure to $15 \mathrm{mg} / \mathrm{L}, 30 \mathrm{mg} / \mathrm{L}$ and $50 \mathrm{mg} / \mathrm{L}$ of nicotine was paired with an environment zebrafish experienced as innately aversive (Kedikian et al., 2013). Despite this, zebrafish became 
conditioned to spend significantly more time in the aversive environment following exposure to all concentrations of nicotine. The reason for the overall lack of a CPP response to acute and chronic nicotine in the present study and the differential results from what has been reported in the literature is unclear. This may be due to genetic variability of zebrafish, differences in apparatus designs, and/or differences in sample sizes, and future investigation is warranted.

Potential bias towards one environment over the other was evaluated in both apparatus designs. An unbiased apparatus allows for a better detection of rewarding or aversive properties and has been held in higher regard than the biased design, as previously discussed (Sanchis-segura \& Spanagel, 2006). In apparatus design 1, significantly more zebrafish displayed a baseline side preference for the white side $(\mathrm{n}=$ 75) than the dotted side $(n=48)$. However, zebrafish spent a comparable amount of time in their preferred side (i.e. white or dotted) during baseline preference testing. The experimenter was concerned that these results indicated a degree of side bias, and decided to create another apparatus design with comparable features of each environment, but distinct enough for zebrafish to discriminate between the two sides. Side bias was not found to be present in zebrafish tested in apparatus design 2, and zebrafish spent a similar amount of time in their preferred side during baseline testing. Thus, apparatus 2 is inferred to be unbiased, in that zebrafish do not display a significant preference for one environment over the other.

Lastly, the effect of conditioning order the change in place preference was assessed. Zebrafish were conditioned in a counter-balanced order, in that half of the animals first received drug on their non-preferred side and received water on their 
preferred side, and the other half of animals first received water on their preferred side and drug on their non-preferred side secondly. Zebrafish that received drug second displayed a significantly greater change in place preference than fish that received drug first. In this latter group, it is likely that the drug is still present in the zebrafish CNS when it is placed into the preferred side second and is given water, after having previously received drug. Pilot studies are currently being conducted in which conditioning is carried out over a period of two days instead of one to avoid such a carryover effect.

\section{Limitations}

There are, however, notable limitations of the zebrafish model of drug reward. One such issue pertains to methods of drug delivery. The most commonly employed method of administration is via submersion in a bath solution containing a concentration of the drug to be absorbed by the gills, skin, and mouth. Zebrafish are known to absorb most water-soluble drugs administered in this manner, but the degree of uptake can vary among individuals (Best \& Alderton, 2008). Conducting preliminary studies to confirm that rates of absorption reflect drug concentration in the water may circumvent this issue. Behavioral paradigms employed in addiction research have only recently been adopted in zebrafish research, and there is thus a lack of information available regarding drug absorption and metabolism rates (Klee, Ebbert, Schneider, Hurt, \& Ekker, 2011). It is possible that the effects of alcohol, nicotine and caffeine may have been influenced by the bioavailability of each substance in the CNS of the zebrafish. Another method of drug administration in zebrafish is intraperitoneal injection, which has been reported to be a more precise method of drug delivery (Kinkel, Eames, Philipson, \& Prince, 2010), 
although injections will reduce the rate of throughput and may be a stressful procedure for animals.

Furthermore, although zebrafish have similar CNS structure to humans and possess all the major mammalian neurotransmitters, there are undoubtedly very large differences in animal physiology. For instance, two forms of the serotonin transporter, SERT A and B, are found in zebrafish and not in mammals or humans (Norton, Folchert, \& Bally-Cuif, 2008; Wang, Takai, Yoshioka, \& Shirabe, 2006). Moreover, as there are notable differences in neuronal architecture, the underlying mechanisms and brain structures associated with reward learning are likely to differ to some degree (Eddins, Petro, Williams, Cerutti, \& Levin, 2009). Experimental subjects used in this study were of a randomly bred genetically heterogeneous background referred to as wildtype. Testing various strains and mutant fish in the CPP paradigm would help shed light on how drug reward is mediated by genetic makeup (Klee et al., 2012; Ninkovic \& BallyCuif, 2006). Another limitation of the present study is low sample sizes, particularly of zebrafish tested in apparatus 2, most notably those that received chronic treatment, which may have reduced the power to detect a true treatment effect. Increasing sample sizes in a number of groups may thus be warranted, and will be carried out in future studies. As previously mentioned, apparatus 2 was designed to eliminate side bias by creating environments that were similar, in that they both possessed blue shapes of the same quantity, but different in pattern and shape. It may be that zebrafish were not able to differentiate between these two environments very well, potentially impacting the ability of these animals to form a strong conditioned association and a CPP response. Future 
studies will be carried out with apparatus designs with a greater degree of environmental distinction, whilst avoiding the creation of a side bias.

\section{Conclusion}

Overall, zebrafish are an excellent animal model for studying human brain disorders, due to an ideal balance of simplicity and complexity in both anatomy and behavior. Conditioned place preference models of drug reward will help illuminate processes and mechanisms underlying the rewarding effects of drugs. Information garnered from zebrafish in this regard, can indicate appropriate avenues of research that would benefit from further investigation in mammalian models, and ultimately, humans. In summary, zebrafish are an excellent model to study the rewarding effects of both wellclassified and novel compounds in a relatively medium to high-throughput manner. This claim is supported by conditioned place preference behavior reported in zebrafish following administration of a wide range psychoactive substances that mirror mammalian CPP findings (Lau et al., 2006; Ninkovic et al., 2006; Braida et al., 2007; Kily et al., 2008; Mathur et al., 2011a; Darland et al., 2012), including those reported here. Information garnered from this study provides further support that the marriage of CPP and zebrafish is a viable model of drug reward, and is sensitive to three frequently used substances, alcohol, caffeine and nicotine. The zebrafish CPP model has intrinsic translational value, and is well-suited for future studies of pharmacological, environmental, and genetic manipulation, which will likely increase understanding of factors contributing to the pathogenesis of addiction and subsequently aid in the develop of treatment and prevention strategies that will contribute to the reduction of human suffering. 
APPENDIX A

INSTITUTIONAL ANIMAL CARE AND USE COMMITTEE NOTICE OF

COMMITTEE ACTION

II
THIIII SOUNIVERSITY OF
SOUTHRN MISSISSIPPI.

INSTITUTIONAL ANIMAL CARE AND USE COMMITIEE

118 College Drive 15116 | Hattiesburg, MS 39406-0001

Phone: 601.266 .4063 | Fax: 601.266.4377 | iacuc@usm.edu | www.usm.edu/acuc

INSTITUTIONAL ANIMAL CARE AND USE COMMITTEE NOTICE OF COMMITTEE ACTION

The proposal noted below was reviewed and approved by The University of Southern Mississippi Institutional Animal Care and Use Committee (IACUC) in accordance with regulations by the United States Department of Agriculture and the Public Health Service Office of Laboratory Animal Welfare. The project expiration date is noted below. If for some reason the project is not completed by the end of the three year approval period, your protocol must be reactivated (a new protocol must be submitted and approved) before further work involving the use of animals can be done.

Any significant changes (see attached) should be brought to the attention of the committee at the earliest possible time. If you should have any questions, please contact me.

PROTOCOL NUMBER:

PROJECT TITLE:

PROPOSED PROJECT DATES:

PROJECT TYPE:

PRINCIPAL INVESTIGATOR(S):

DEPARTMENT:

FUNDING AGENCY/SPONSOR:

IACUC COMMITTEE ACTION:

PROTOCOL EXPIBATON DATE:

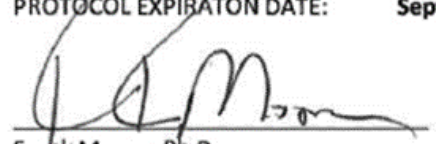

Frahk Moore, Ph.D.

IACUC Chair
14021302

Conditioned Place Preference Behavior in Zebrafish (Danio Rerio) 2/2014-9/2016

New

David Echevarria

Phychology

Full Committee Approval

September 30, 2016

Date 


\section{APPENDIX B}

\section{RELATED PUBLICATIONS}

CE: Pratima ED: Maitreyee Op. csr FBP 200706: LWW_FBP_200706 The utility of the zebrafish model in conditioned place
preference to assess the rewarding effects of drugs Adam D. Collier and David J. Echevarria

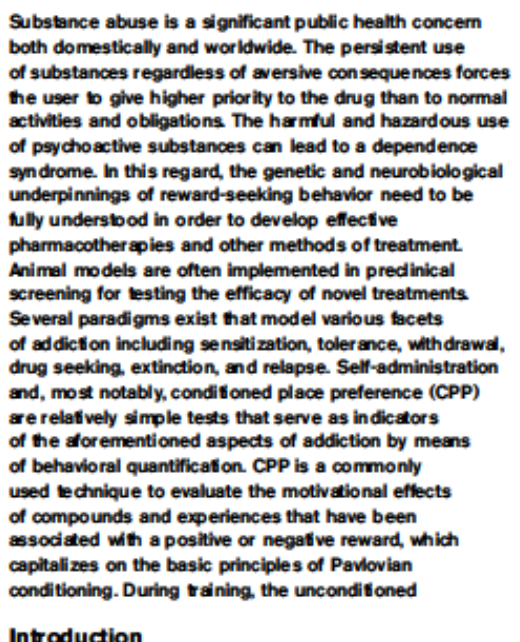

Introduction

Substance abuse has become a significant public health concem with widespread detrimental consequences both domestically and worldwidc. In 2011, an extimated 21.6 million Americans (8.4\% of the population) who were 12 years and older needed treatment for problems associated with alcohol andor drugs, with only 2.3 million recciving it (National Institute on Drug Abusc). The abuse of tobacce, alcohol, and illicit drugs not only causes a host of health issues for the drug user but also accrues $\sim \$ 600$ billion of health, crime, and productivity-felated expendinures annually in the USA alone (National Institute on Drug Abuse). In an effort to assess the abuse potential of addictive substances, it is imperative that genetic and neu robiological mechanisms underlying reward-secking behavior be fully understood. Adoption, family, and twin studics have found that substance buse and dependence are heritable traits (Agrawal and Lynskey, 2008). Thus, the identification of candidate genes, in addition to gaining a solid understanding of the neurobiological basis of reward sceking, would facilitate development of new pharmacotherapies Animal models have been used in this cndeavor.

Laboratory animal behavioral paradigms have been used to model various facets of addiction, including sensitization,

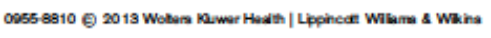

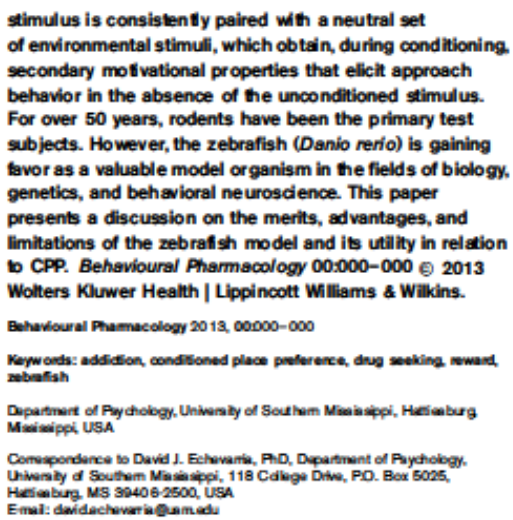

Heceived 30 March 2013 Accopted as metsed 20 May 2013

tolerance, withdrawal, extinction, and relapse (Mohn at ad, 2004). Animal behavior that can be essily quantified has allowed self-administration and conditioned place preference assays to serve as indicators of the aforementioned faces of addiction. Traditionally, rats and mice have been the go-to laboratory animals when modeling human disease states, principally because of the anatomical, biological, and genomic homology between rodents and humans (Lieschke and Curric, 2007). However, the rodent model is hindered by a number of feanues, including high cose, disced husbandry, lenghy developmental periods, and inefficiency in high-throughput techniques.

The zebrafish (Davio revio), compensating for these disadvantages, has been accepted as a valuable mode organism in the fields of developmental biology and genetics and is steadily gaining popularity in behavioral neuroscience. Although there is a degree of physiological and phylogenetic disparity between fish and humans, the eentral nervous system (CNS) of aebrafish develops and is organized in a similar manner to that of their fellow vertebrates, and analogous circuitry that mediates reward has been identificd in the zebrafish brain (Rink and Wullimann, 2002). Furthet, the zebrafish genome has been fully sequenced (Postlethwait et al, 1998; Woods DOE 101007/FP.06013:328363414a 


\section{RELATED PUBLICATIONS}

Progress in Neuro-Pyychopharmacology \& Biological Pyychiatry 55 (2014) 16-25

\begin{tabular}{cc|}
\hline & Contents lists available at ScienceDirect \\
& Progress in Neuro-Psychopharmacology \& Biological \\
ELSEVIER & PSychiatry \\
\hline
\end{tabular}

Zebrafish and conditioned place preference: A translational model of drug reward

Adam D. Collier ${ }^{\text {a }}$, Kanza M. Khan ${ }^{\text {a }}$, Erika M. Caramillo ${ }^{a}$, Richard S. Mohn ${ }^{\text {b }}$, David J. Echevarria a,*

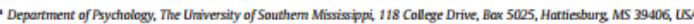

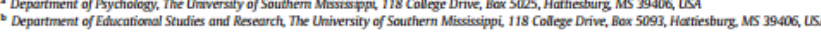

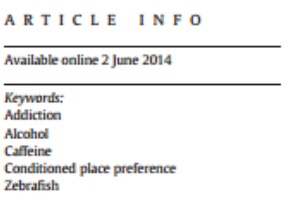

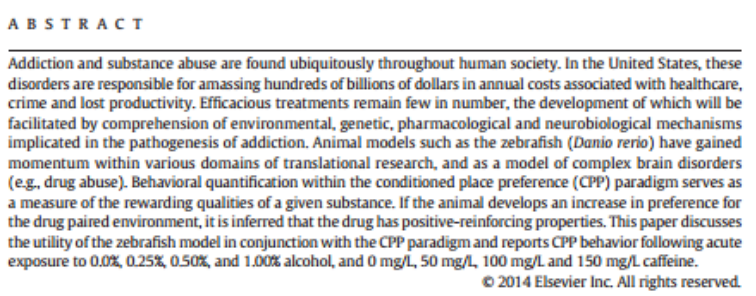

1. Introduction

Substance abuse and addiction are complex brain disorders found ubiquitously throughout human society; they not only cause tremendous harm to the user, but are estimated to amass hundreds of billions of dollars annually in costs associated with health care, crime, and lost productivity, solely within the United States (National Institute on Drug Abuse (NIDA], 2010). Alcohol is a particularly devastating substance; it's the third largest risk factor for disease in the world and is responsible for -2.5 millisk deaths annlly (World Health Organization [WHO], 2011). Globally, the annual use of alcohol is estimated to be cight tiwes higher than the and Cafferalence of ilicit drug use (UNODC World Drug Report, 2012). Caffeine, a less harmful substance than alcohol, does not meet the criteria necessary to elicit sub-

Abrreviations: NDA, national institute on drug abuse; WHO, worlid health organizer. fion: UNODC, united nations office of druzs and crime; DSM-V, diagnostic and statistical CNS, central nervous system; GABA, gamma-iminobutyric aid; CPP, conditioned place preference; CPA, conditioned place aversion; IP, intraperitoneal; : MM, intramuscular; UCS, unconditioned stimalus; CS, conditioned stimulus; ANOVA, anulysis of variance; VTA ventex; BAC, blood alcohol concentration

Coxresponding author. Tel: + 16012665724

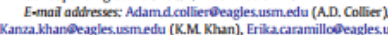

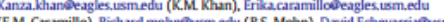
(D. Echevarria). drug in the world (Winston, 2005) with over $85 \%$ of people consuming it regularly (American Psychiatric Association, 2013)

Efficacious treatments for substance abuse remain few in number. Development of novel pharmacotherapies and prevention/intervention strategies will be facilitated by comprehension of environmental, genetic pharmacological and neurobiological mechanisms implicated in the pathogenesis of addiction. Animal models have been utilized to help elcidate such meetanisns and processes, particularly those associated with the experience of reward. Organism survival is often dependent upon leaning the conditions necessary to acquire natirally rewarding and reinforcing stimuli that serve homeostatic and reproductive purposes (Hyman et al., 2006). Animals rapidly learn the behaviora responses necessary to obtain natural rewards (e.g. food or sex) and 2006 ). 2006). Leaming also occurs following consumption of rewarding psychaactive substances (Everitt et al., 2001, Hyman et al., 2006).

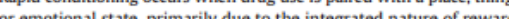
or entional state, primarily due to the integrated nature of reward limbic sytem (Malellan a 2000). Exposure to the aforenetion

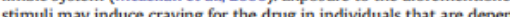
stimu anace craving for the drug in holve

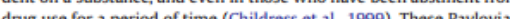
drug use for a period of time (Childress et al., 1999). These Pavlovian and operant leaking processes are believed to juat ate transtions from casual, voluntary drug use, to more habiual and compulsive standing how such factors contribute to drug seeking behaviors 


\section{REFERENCES}

Almela, P., Navarro-Zaragoza, J., García-Carmona, J. A., Mora, L., Hidalgo, J., Milanés, M. V., \& Laorden, M. L. (2012). Role of corticotropin-releasing factor (CRF) receptor-1 on the catecholaminergic response to morphine withdrawal in the nucleus accumbens (NAc). PloS One, 7(10), e47089. doi:10.1371/journal.pone.0047089

American Psychiatric Association. (2013). Diagnostic and statistical manual of mental disorders (5th ed.). Arlington, VA: American Psychiatric Publishing.

Anderson, B. L., \& Juliano, L. M. (2012). Behavior, sleep, and problematic caffeine consumption in a college-aged sample. Journal of Caffeine Research, 2(1), 38-44. doi:10.1089/jcr.2012.0009

Bahi, A., Kusnecov, A. W., \& Dreyer, J. L. (2008). Effects of urokinase-type plasminogen activator in the acquisition, expression and reinstatement of cocaineinduced conditioned-place preference. Behavioural Brain Research, 191(1), 17-25. doi:10.1016/j.bbr.2008.03.004

Bardo, M. T., \& Bevins, R. A. (2000). Conditioned place preference: what does it add to our preclinical understanding of drug reward? Psychopharmacology, 153(1), 31-43. doi: $10.1007 / \mathrm{s} 002130000569$

Bell, M. R., Meerts, S. H., \& Sisk, C. L. (2010). Male Syrian hamsters demonstrate a conditioned place preference for sexual behavior and female chemosensory stimuli. Hormones and Behavior, 58(3), 410-4. doi:10.1016/j.yhbeh.2010.05.017

Bernabeu, R. O., Aires, B., \& Behavior, A. (2013). Behavioral and molecular analysis of nicotine-conditioned place preference in zebrafish Behavioral and molecular analysis of nicotine-conditioned place. PloS One, 8(7). 
Best, J. D., \& Alderton, W. K. (2008). Zebrafish: An in vivo model for the study of neurological diseases. Neuropsychiatric Disease and Treatment, 4(3), 567-76.

Braida, D., Limonta, V., Pegorini, S., Zani, A., Guerini-Rocco, C., Gori, E., \& Sala, M. (2007). Hallucinatory and rewarding effect of salvinorin A in zebrafish: kappaopioid and CB1-cannabinoid receptor involvement. Psychopharmacology, 190(4), 441-8. doi:10.1007/s00213-006-0639-1

Braida, D., Pozzi, M., Cavallini, R., \& Sala, M. (2001). Conditioned place preference induced by the cannabinoid agonist CP 55,940: interaction with the opioid system. Neuroscience, 104(4), 923-926. doi:10.1016/S0306-4522(01)00210-X

Brielmaier, J. M., McDonald, C. G., \& Smith, R. F. (2008). Nicotine place preference in a biased conditioned place preference design. Pharmacology, Biochemistry, and Behavior, 89(1), 94-100. doi:10.1016/j.pbb.2007.11.005

Cachat, J., Canavello, P., Elegante, M., Bartels, B., Hart, P., Bergner, C., ... Kalueff, A. V. (2010). Modeling withdrawal syndrome in zebrafish. Behavioural Brain Research, 208(2), 371-6. doi:10.1016/j.bbr.2009.12.004

Cachat, J., Kyzar, E. J., Collins, C., Gaikwad, S., Green, J., Roth, A., ... Kalueff, A. V. (2013). Unique and potent effects of acute ibogaine on zebrafish: the developing utility of novel aquatic models for hallucinogenic drug research. Behavioural Brain Research, 236(1), 258-69. doi:10.1016/j.bbr.2012.08.041

Cachat, J., Stewart, A., Grossman, L., Gaikwad, S., Kadri, F., Chung, K. M., ... Kalueff, A. V. (2010). Measuring behavioral and endocrine responses to novelty stress in adult zebrafish. Nature Protocols, 5(11), 1786-99. doi:10.1038/nprot.2010.140 
Capiotti, K. M., Menezes, F. P., Nazario, L. R., Pohlmann, J. B., de Oliveira, G. M. T., Fazenda, L., ... Da Silva, R. S. (2011). Early exposure to caffeine affects gene expression of adenosine receptors, DARPP-32 and BDNF without affecting sensibility and morphology of developing zebrafish (Danio rerio). Neurotoxicology and Teratology, 33(6), 680-5. doi:10.1016/j.ntt.2011.08.010

Carelli, R. M., Williams, J. G., \& Hollander, J. A. (2003). Basolateral amygdala neurons encode cocaine self-administration and cocaine-associated cues. The Journal of Neuroscience $\square$ : The Official Journal of the Society for Neuroscience, 23(23), 820411. Retrieved from http://www.ncbi.nlm.nih.gov/pubmed/12967981

Cauli, O., \& Morelli, M. (2005). Caffeine and the dopaminergic system. Behavioural Pharmacology, 16(2), 63-77. Retrieved from http://www.ncbi.nlm.nih.gov/pubmed/15767841

Centers for Disease Control (2015). Smoking \& Tobacco Use. Retrieved from http://www.cdc.gov/tobacco/

Chacon, D. M., \& Luchiari, A. C. (2014). A dose for the wiser is enough: The alcohol benefits for associative learning in zebrafish. Progress in NeuroPsychopharmacology \& Biological Psychiatry, 53C, 109-115. doi:10.1016/j.pnpbp.2014.03.009

Chatterjee, D., \& Gerlai, R. (2009). High precision liquid chromatography analysis of dopaminergic and serotoninergic responses to acute alcohol exposure in zebrafish. Behavioural Brain Research, 200(1), 208-213. doi:10.1016/j.bbr.2009.01.016

Childress, A. R., Mozley, P. D., McElgin, W., Fitzgerald, J., Reivich, M., \& O’Brien, C. P. (1999). Limbic Activation During Cue-Induced Cocaine Craving. American 
Journal of Psychiatry, 156(1), 11-18. Retrieved from

http://journals.psychiatryonline.org/article.aspx?articleid=173211

Collier, A. D., \& Echevarria, D. J. (2013). The utility of the zebrafish model in conditioned place preference to assess the rewarding effects of drugs. Behavioural Pharmacology, 24(5-6), 375-83. doi:10.1097/FBP.0b013e328363d14a

Collier, A. D., Khan, K. M., Caramillo, E. M., Mohn, R. S., \& Echevarria, D. J. (2014). Zebrafish and conditioned place preference: a translational model of drug reward. Progress in Neuro-Psychopharmacology \& Biological Psychiatry, 55, 16-25. doi:10.1016/j.pnpbp.2014.05.014

Colwill, R. M., Raymond, M. P., Ferreira, L., \& Escudero, H. (2005). Visual discrimination learning in zebrafish (Danio rerio). Behavioural Processes, 70(1), 19-31. doi:10.1016/j.beproc.2005.03.001

Crabbe, J. C., Belknap, J. K., \& Buck, K. J. (1994). Genetic animal models of alcohol and drug abuse. Science (New York, N.Y.), 264(5166), 1715-23. Retrieved from http://www.ncbi.nlm.nih.gov/pubmed/8209252

Cunningham, C. L., Ferree, N. K., \& Howard, M. A. (2003). Apparatus bias and place conditioning with ethanol in mice. Psychopharmacology, 170(4), 409-22. doi:10.1007/s00213-003-1559-y

Darland, T., \& Dowling, J. E. (2001). Behavioral screening for cocaine sensitivity in mutagenized zebrafish. Proceedings of the National Academy of Sciences of the United States of America, 98(20), 11691-6. doi:10.1073/pnas.191380698

Darland, T., Mauch, J. T., Meier, E. M., Hagan, S. J., Dowling, J. E., \& Darland, D. C. (2012). Sulpiride, but not SCH23390, modifies cocaine-induced conditioned place 
preference and expression of tyrosine hydroxylase and elongation factor $1 \alpha$ in zebrafish. Pharmacology, Biochemistry, and Behavior, 103(2), 157-67. doi:10.1016/j.pbb.2012.07.017

Di Ciano, P., Blaha, C. D., \& Phillips, A. G. (1998). Conditioned changes in dopamine oxidation currents in the nucleus accumbens of rats by stimuli paired with selfadministration or yoked-administration of d-amphetamine. The European Journal of Neuroscience, 10(3), 1121-7. Retrieved from http://www.ncbi.nlm.nih.gov/pubmed/9753180

Dlugos, C. A., \& Rabin, R. A. (2003). Ethanol effects on three strains of zebrafish: model system for genetic investigations. Pharmacology, Biochemistry, and Behavior, 74(2), 471-80. Retrieved from http://www.ncbi.nlm.nih.gov/pubmed/12479969

Driever, W., Solnica-Krezel, L., Schier, A. F., Neuhauss, S. C., Malicki, J., Stemple, D. L., ... Boggs, C. (1996). A genetic screen for mutations affecting embryogenesis in zebrafish. Development, 123, 37-46. Retrieved from http://www.ncbi.nlm.nih.gov/pubmed/9007227

Echevarria, D. J., Buske, C., Toms, C. N., \& Jouandot, D. J. (2011). Zebrafish Neurobehavioral Protocols. In A. V. Kalueff \& J. M. Cachat (Eds.). Neuromethods (Vol. 51). Totowa, NJ: Humana Press. doi:10.1007/978-1-60761-953-6

Echevarria, D. J., Hammack, C. M., Jouandot, D. J., \& Toms, C. N. (2010). Does Acute Alcohol Exposure Modulate Aggressive Behaviors in the Zebrafish (Danio rerio), or is the Bark Worse than the Bite? International Journal of Comparative Psychology, $23,62-69$. 
Eddins, D., Petro, A., Williams, P., Cerutti, D. T., \& Levin, E. D. (2009). Nicotine effects on learning in zebrafish: the role of dopaminergic systems. Psychopharmacology, 202(1-3), 103-9. doi:10.1007/s00213-008-1287-4

Egan, R. J., Bergner, C. L., Hart, P. C., Cachat, J. M., Canavello, P. R., Elegante, M. F., ... Kalueff, A. V. (2009). Understanding behavioral and physiological phenotypes of stress and anxiety in zebrafish. Behavioural Brain Research, 205(1), 38-44. doi:10.1016/j.bbr.2009.06.022

El Yacoubi, M., Ledent, C., Parmentier, M., Costentin, J., \& Vaugeois, J. M. (2000). The anxiogenic-like effect of caffeine in two experimental procedures measuring anxiety in the mouse is not shared by selective $\mathrm{A}(2 \mathrm{~A})$ adenosine receptor antagonists.

Psychopharmacology, 148(2), 153-63. Retrieved from http://www.ncbi.nlm.nih.gov/pubmed/10663430

Engeszer, R. E., Patterson, L. B., Rao, A. A, \& Parichy, D. M. (2007). Zebrafish in the wild: a review of natural history and new notes from the field. Zebrafish, 4(1), 2140. doi:10.1089/zeb.2006.9997

Everitt, B. J., Dickinson, A., \& Robbins, T. W. (2001). The neuropsychological basis of addictive behaviour. Brain Research Reviews, 36(2-3), 129-138. doi:10.1016/S0165-0173(01)00088-1

Ferré, S., Fuxe, K., von Euler, G., Johansson, B., \& Fredholm, B. B. (1992). Adenosinedopamine interactions in the brain. Neuroscience, 51(3), 501-12. Retrieved from http://www.ncbi.nlm.nih.gov/pubmed/1488111

Ferre, S., von Euler, G., Johansson, B., Fredholm, B. B., \& Fuxe, K. (1991). Stimulation of high-affinity adenosine A2 receptors decreases the affinity of dopamine D2 
receptors in rat striatal membranes. Proceedings of the National Academy of Sciences of the United States of America, 88(16), 7238-41. Retrieved from http://www.pubmedcentral.nih.gov/articlerender.fcgi?artid=52269\&tool=pmcentrez \&rendertype $=$ abstract

Fredholm, B. B., Bättig, K., Holmén, J., Nehlig, A., \& Zvartau, E. E. (1999). Actions of caffeine in the brain with special reference to factors that contribute to its widespread use. Pharmacological Reviews. Retrieved from http://www.scopus.com/inward/record.url?eid=2-s2.00345561561\&partnerID=tZOtx3y1

Garrett, B. E., \& Griffiths, R. R. (1997). The role of dopamine in the behavioral effects of caffeine in animals and humans. Pharmacology, Biochemistry, and Behavior, 57(3), 533-41. Retrieved from http://www.ncbi.nlm.nih.gov/pubmed/9218278

Gerlai, R., Lahav, M., Guo, S., \& Rosenthal, A. (2000). Drinks like a fish: zebra fish (Danio rerio) as a behavior genetic model to study alcohol effects. Pharmacology, Biochemistry, and Behavior, 67(4), 773-82. Retrieved from http://www.ncbi.nlm.nih.gov/pubmed/11166068

Gerlai, R., Lee, V., \& Blaser, R. (2006). Effects of acute and chronic ethanol exposure on the behavior of adult zebrafish (Danio rerio). Pharmacology, Biochemistry, and Behavior, 85(4), 752-61. doi:10.1016/j.pbb.2006.11.010

Goeders, N. E., \& Guerin, G. F. (1996). Role of corticosterone in intravenous cocaine self-administration in rats. Neuroendocrinology, 64(5), 337-48. Retrieved from http://www.ncbi.nlm.nih.gov/pubmed/8930934 
Gould, G. G. (2011). Modified associative learning T-maze test for zebrafish ( Danio rerio) and other small teleost fish. In A. V. Kalueff \& J. M. Cachat (Eds.), Neuromethods (51st ed., pp. 61-73). New York: Humana Press.

Grossman, L., Utterback, E., Stewart, A., Gaikwad, S., Chung, K. M., Suciu, C., ... Kalueff, A. V. (2010). Characterization of behavioral and endocrine effects of LSD on zebrafish. Behavioural Brain Research, 214(2), 277-84. doi:10.1016/j.bbr.2010.05.039

Guo, S. (2009). Using zebrafish to assess the impact of drugs on neural development and function. Expert Opinion on Drug Discovery, 4(7), 715-726. doi: $10.1517 / 17460440902988464$

Haffter, P., Granato, M., Brand, M., Mullins, M. C., Hammerschmidt, M., Kane, D. A., ... Nüsslein-Volhard, C. (1996). The identification of genes with unique and essential functions in the development of the zebrafish, Danio rerio. Development (Cambridge, England), 123, 1-36. Retrieved from http://www.ncbi.nlm.nih.gov/pubmed/9007226

Howe, K., Clark, M. D., Torroja, C. F., Torrance, J., Berthelot, C., Muffato, M., ... Schuster, S. C. (2013). The zebrafish reference genome sequence and its relationship to the human genome. Nature, 496(7446), 498-503. doi:10.1038/nature12111

Hyman, S. E., Malenka, R. C., \& Nestler, E. J. (2006). Neural mechanisms of addiction: the role of reward-related learning and memory. Annual Review of Neuroscience, 29, 565-98. doi:10.1146/annurev.neuro.29.051605.113009 
Juliano, L. M., \& Griffiths, R. R. (2004). A critical review of caffeine withdrawal: empirical validation of symptoms and signs, incidence, severity, and associated features. Psychopharmacology, 176(1), 1-29. doi:10.1007/s00213-004-2000-x

Kalueff, A. V, Gebhardt, M., Stewart, A. M., Cachat, J. M., Brimmer, M., Chawla, J. S., ... Schneider, H. (2013). Towards a comprehensive catalog of zebrafish behavior 1.0 and beyond. Zebrafish, 10(1), 70-86. doi:10.1089/zeb.2012.0861

Karnik, I., \& Gerlai, R. (2012). Can zebrafish learn spatial tasks? An empirical analysis of place and single CS-US associative learning. Behavioural Brain Research, 233(2), 415-21. doi:10.1016/j.bbr.2012.05.024

Kedikian, X., Faillace, M. P., \& Bernabeu, R. (2013). Behavioral and molecular analysis of nicotine-conditioned place preference in zebrafish. PloS One, 8(7), e69453. doi:10.1371/journal.pone.0069453

Kily, L. J. M., Cowe, Y. C. M., Hussain, O., Patel, S., McElwaine, S., Cotter, F. E., \& Brennan, C. H. (2008). Gene expression changes in a zebrafish model of drug dependency suggest conservation of neuro-adaptation pathways. The Journal of Experimental Biology, 211(Pt 10), 1623-34. doi:10.1242/jeb.014399

Kinkel, M. D., Eames, S. C., Philipson, L. H., \& Prince, V. E. (2010). Intraperitoneal injection into adult zebrafish. Journal of Visualized Experiments $\square:$ JoVE, (42). doi: $10.3791 / 2126$

Klee, E. W., Ebbert, J. O., Schneider, H., Hurt, R. D., \& Ekker, S. C. (2011). Zebrafish for the study of the biological effects of nicotine. Nicotine \& Tobacco Research, 13(5), 301-12. doi:10.1093/ntr/ntr010 
Klee, E. W., Schneider, H., Clark, K. J., Cousin, M. a, Ebbert, J. O., Hooten, W. M., ... Ekker, S. C. (2012). Zebrafish: a model for the study of addiction genetics. Human Genetics, 131(6), 977-1008. doi:10.1007/s00439-011-1128-0

Koob, G. F., \& Volkow, N. D. (2010). Neurocircuitry of addiction. Neuropsychopharmacology, 35(1), 217-38. doi:10.1038/npp.2009.110

Kotlinska, J. H., Bochenski, M., \& Danysz, W. (2011). The role of group I mGlu receptors in the expression of ethanol-induced conditioned place preference and ethanol withdrawal seizures in rats. European Journal of Pharmacology, 670(1), 154-61. doi:10.1016/j.ejphar.2011.09.025

Kyzar, E. J., Collins, C., Gaikwad, S., Green, J., Roth, A., Monnig, L., ... Kalueff, A. V. (2012). Effects of hallucinogenic agents mescaline and phencyclidine on zebrafish behavior and physiology. Progress in Neuro-Psychopharmacology \& Biological Psychiatry, 37(1), 194-202. doi:10.1016/j.pnpbp.2012.01.003

Lardelli, M. (2000). Zebrafish - Do we need another vertebrate model? Anzccart News, 13(4).

Larimer, M. E., Palmer, R. S., \& Marlatt, G. A. (1999). Relapse prevention. An overview of Marlatt's cognitive-behavioral model. Alcohol Research \& Health, 23(2), 15160. Retrieved from http://www.ncbi.nlm.nih.gov/pubmed/10890810

Lau, B., Bretaud, S., Huang, Y., Lin, E., \& Guo, S. (2006). Dissociation of food and opiate preference by a genetic mutation in zebrafish. Genes, Brain, and Behavior, 5(7), 497-505. doi:10.1111/j.1601-183X.2005.00185.x

Li, F., Fang, Q., Liu, Y., Zhao, M., Li, D., Wang, J., \& Lu, L. (2008). Cannabinoid CB(1) receptor antagonist rimonabant attenuates reinstatement of ketamine conditioned 
place preference in rats. European Journal of Pharmacology, 589(1-3), 122-6. doi:10.1016/j.ejphar.2008.04.051

Liang, J., Li, Y., Ping, X., Yu, P., Zuo, Y., Wu, L., .. Cui, C. (2006). The possible involvement of endogenous ligands for mu-, delta- and kappa-opioid receptors in modulating morphine-induced CPP expression in rats. Peptides, 27(12), 3307-14. doi:10.1016/j.peptides.2006.08.011

Lieschke, G. J., \& Currie, P. D. (2007). Animal models of human disease: zebrafish swim into view. Nature Reviews. Genetics, 8(5), 353-67. doi:10.1038/nrg2091

López-Patiño, M., Yu, L., Yamamoto, B. K., \& Zhdanova, I. V. (2008). Gender differences in zebrafish responses to cocaine withdrawal. Physiology \& Behavior, 95(1-2), 36-47. doi:10.1016/j.physbeh.2008.03.021

Manuel, R., Gorissen, M., Piza Roca, C., Zethof, J., Vis, H. van de, Flik, G., \& Bos, R. (2014). Inhibitory Avoidance Learning in Zebrafish (Danio Rerio): Effects of shock intensity and unraveling differences in task performance. Zebrafish. doi:10.1089/zeb.2013.0970

Marlatt, G. A., \& Donovan, D. M. (2005). Relapse Prevention: Maintenance Strategies in the Treatment of Addictive Behaviors. Retrieved from http://books.google.com/books?hl=en\&lr=\&id=lh17mhpxPBYC\&pgis=1

Mathur, P., Berberoglu, M. A, \& Guo, S. (2011). Preference for ethanol in zebrafish following a single exposure. Behavioural Brain Research, 217(1), 128-33. doi:10.1016/j.bbr.2010.10.015 
Mathur, P., \& Guo, S. (2010). Use of zebrafish as a model to understand mechanisms of addiction and complex neurobehavioral phenotypes. Neurobiology of Disease, 40(1), 66-72. doi:10.1016/j.nbd.2010.05.016

Mathur, P., Lau, B., \& Guo, S. (2011). Conditioned place preference behavior in zebrafish. Nature Protocols, 6(3), 338-45. doi:10.1038/nprot.2010.201

Maximino, C., Lima, M. G., Olivera, K. R. M., Picanço-Diniz, D. L. W., \& Herculano, A. M. (2011). Adenosine A1, but not A2, receptor blockade increases anxiety and arousal in Zebrafish. Basic \& Clinical Pharmacology \& Toxicology, 109(3), 203-7. doi:10.1111/j.1742-7843.2011.00710.x

McLellan, A. T., Lewis, D. C., O’Brien, C. P., \& Kleber, H. D. (2000). Drug dependence, a chronic medical illness implications for treatment, insurance, and outcomes evaluation. Journal of the American Medical Association, 284(13), 1689-1695.

Retrieved from http://www.scopus.com/inward/record.url?eid=2-s2.00034605531\&partnerID=tZOtx3y1

Miller, N. Y., \& Gerlai, R. (2011). Shoaling in zebrafish: what we don't know. Reviews in the Neurosciences, 22(1), 17-25. doi:10.1515/RNS.2011.004

Nehlig, A. (1999). Are we dependent upon coffee and caffeine? A review on human and animal data. Neuroscience and Biobehavioral Reviews, 23(4), 563-76. Retrieved from http://www.ncbi.nlm.nih.gov/pubmed/10073894

Nicotine Addiction: Past and present. (2010). Centers for Disease Control and Prevention (US). Retrieved from http://www.ncbi.nlm.nih.gov/books/NBK53018/ 
National Institute on Drug Abuse. (2014). DrugFacts: Cigarettes and other tobacco products. Retrieved from http://www.drugabuse.gov/publications/drugfacts/cigarettes-other-tobacco-products

Ninkovic, J., \& Bally-Cuif, L. (2006). The zebrafish as a model system for assessing the reinforcing properties of drugs of abuse. Methods, 39(3), 262-74.

doi:10.1016/j.ymeth.2005.12.007

Ninkovic, J., Folchert, A., Makhankov, Y. V, Neuhauss, S. C. F., Sillaber, I., Straehle, U., \& Bally-cuif, L. (2006). Genetic Identification of AChE as a positive modulator of addiction to the psychostimulant d-amphetamine in zebrafish. Journal of Neurobiology, 5, 463-475. doi:10.1002/neu

Norton, W. J., Folchert, A., \& Bally-Cuif, L. (2008). Comparative analysis of serotonin receptor (HTR1A/HTR1B families) and transporter (slc6a4a/b) gene expression in the zebrafish brain. The Journal of Comparative Neurology, 511(4), 521-42. doi:10.1002/cne.21831

Pan, Y., Chatterjee, D., \& Gerlai, R. (2012). Strain dependent gene expression and neurochemical levels in the brain of zebrafish: focus on a few alcohol related targets. Physiology \& Behavior, 107(5), 773-80. doi:10.1016/j.physbeh.2012.01.017

Panula, P., Chen, Y.-C., Priyadarshini, M., Kudo, H., Semenova, S., Sundvik, M., \& Sallinen, V. (2010). The comparative neuroanatomy and neurochemistry of zebrafish CNS systems of relevance to human neuropsychiatric diseases. Neurobiology of Disease, 40(1), 46-57. doi:10.1016/j.nbd.2010.05.010 
Papp, M., Gruca, P., \& Willner, P. (2002). Selective blockade of drug-induced place preference conditioning by ACPC, a functional NDMA-receptor antagonist. Neuropsychopharmacology, 27(5), 727-43. doi:10.1016/S0893-133X(02)00349-4

Parmar, A, Parmar, M, Brennan, C. (2011). Zebrafish conditioned place preference models of drug reinforcement and relapse to drug seeking. In A. V. Kalueff \& J. M. Cachat (Eds.), Zebrafish Neurobehavioral Protocols (Vol. 51, pp. 75-84). New York: Humana Press. doi:10.1007/978-1-60761-953-6

Patkina, N. A., \& Zvartau, E. E. (1998). Caffeine place conditioning in rats: comparison with cocaine and ethanol. European Neuropsychopharmacology, 8(4), 287-291. doi:10.1016/S0924-977X(97)00086-2

Paton, J. J., Belova, M. A., Morrison, S. E., \& Salzman, C. D. (2006). The primate amygdala represents the positive and negative value of visual stimuli during learning. Nature, 439(7078), 865-70. doi:10.1038/nature04490

Peck, C. J., Lau, B., \& Salzman, C. D. (2013). The primate amygdala combines information about space and value. Nature Neuroscience, 16(3), 340-8. doi:10.1038/nn.3328

Pollack, A. E., \& Fink, J. S. (1995). Adenosine antagonists potentiate D2 dopaminedependent activation of Fos in the striatopallidal pathway. Neuroscience, 68(3), 721-8. Retrieved from http://www.ncbi.nlm.nih.gov/pubmed/8577368

Reed, B., \& Jennings, M. (2010). Guidance on the housing and care of Zebrafish Danio rerio. Royal Society for the Prevention of Curelty to Animals.

Rink, E., \& Wullimann, M. F. (2002a). Connections of the ventral telencephalon and tyrosine hydroxylase distribution in the zebrafish brain (Danio rerio) lead to 
identification of an ascending dopaminergic system in a teleost. Brain Research Bulletin, 57(3-4), 385-7. Retrieved from

http://www.ncbi.nlm.nih.gov/pubmed/11922994

Rink, E., \& Wullimann, M. F. (2002b). Development of the catecholaminergic system in the early zebrafish brain: an immunohistochemical study. Brain Research.

Developmental Brain Research, 137(1), 89-100. Retrieved from http://www.ncbi.nlm.nih.gov/pubmed/12128258

Ruhl, N., McRobert, S. P., \& Currie, W. S. (2009). Shoaling preferences and the effects of sex ratio on spawning and aggression in small laboratory populations of zebrafish (Danio rerio). Lab Animal, 38(8), 264-9. doi:10.1038/laban0809-264

Russo, S. J., Sun, W. L., Minerly, A. C. E., Weierstall, K., Nazarian, A., Festa, E. D., ... Quiñones-Jenab, V. (2008). Progesterone attenuates cocaine-induced conditioned place preference in female rats. Brain Research, 1189, 229-35. doi:10.1016/j.brainres.2007.10.057

Sanchis-Segura, C., \& Spanagel, R. (2006). Behavioural assessment of drug reinforcement and addictive features in rodents $\square$ : an overview, 2-38. doi:10.1111/j.1355-6215.2006.00012.x

Sawyer, D. A., Julia, H. L., \& Turin, A. C. (1982). Caffeine and human behavior: Arousal, anxiety, and performance effects. Journal of Behavioral Medicine, 5(4), 415-439. doi:10.1007/BF00845371

Schechter, M. D., \& Calcagnetti, D. J. (1998). Continued trends in the conditioned place preference literature from 1992 to 1996, inclusive, with a cross-indexed 
bibliography. Neuroscience and Biobehavioral Reviews, 22(6), 827-46. Retrieved from http://www.ncbi.nlm.nih.gov/pubmed/9809314

Silva, S. M., \& Madeira, M. D. (2012). Effects of chronic alcohol consumption and withdrawal on the response of the male and female hypothalamic-pituitary-adrenal axis to acute immune stress. Brain Research, 1444, 27-37.

doi:10.1016/j.brainres.2012.01.013

Sison, M., \& Gerlai, R. (2010). Associative learning in zebrafish (Danio rerio) in the plus maze. Behavioural Brain Research, 207(1), 99-104. doi:10.1016/j.bbr.2009.09.043

Spence, R., Gerlach, G., Lawrence, C., \& Smith, C. (2008). The behaviour and ecology of the zebrafish, Danio rerio. Biological Reviews of the Cambridge Philosophical Society, 83(1), 13-34. doi:10.1111/j.1469-185X.2007.00030.x

Stewart, A., Kadri, F., Dileo, J., Chung, K. M., Cachat, J., Goodspeed, J., ... Kalueff, A. V. (2010). The Developing Utility of Zebrafish in Modeling Neurobehavioral Disorders, 104-120.

Stewart, A., Wong, K., Cachat, J., Gaikwad, S., Kyzar, E., Wu, N., ... Kalueff, A. V. (2011). Zebrafish models to study drug abuse-related phenotypes. Reviews in the Neurosciences, 22(1), 95-105. doi:10.1515/RNS.2011.011

Stewart, A., Wu, N., Cachat, J., Hart, P., Gaikwad, S., Wong, K., ... Kalueff, A. V. (2011). Pharmacological modulation of anxiety-like phenotypes in adult zebrafish behavioral models. Progress in Neuro-Psychopharmacology \& Biological Psychiatry, 35(6), 1421-31. doi:10.1016/j.pnpbp.2010.11.035 
Streisinger, G., Walker, C., Dower, N., Knauber, D., \& Singer, F. (1981). Production of clones of homozygous diploid zebra fish (Brachydanio rerio). Nature, 291(5813), 293-296. doi:10.1038/291293a0

Striley, C. W., Griffiths, R. R., \& Cottler, L. B. (2011). Evaluating Dependence Criteria for Caffeine. Journal of Caffeine Research, 1(4), 219-225.

doi:10.1089/jcr.2011.0029

Tran, S., \& Gerlai, R. (2013). Time-course of behavioural changes induced by ethanol in zebrafish (Danio rerio). Behavioural Brain Research, 252, 204-13. doi:10.1016/j.bbr.2013.05.065

Tropepe, V., \& Sive, H. (2003). Can zebrafish be used as a model to study the neurodevelopmental causes of autism? Genes, Brain and Behavior, 268-281. doi:10.1046/j.1601-183X.2003.00038.x

Trotha, J. W., Vernier, P., \& Bally-Cuif, L. (2014). Emotions and motivated behavior converge on an amygdala-like structure in the zebrafish. The European Journal of Neuroscience. doi:10.1111/ejn.12692

Tzschentke, T. M. (1998). Measuring reward with the conditioned place preference paradigm: a comprehensive review of drug effects, recent progress and new issues. Progress in Neurobiology, 56(6), 613-72. Retrieved from http://www.ncbi.nlm.nih.gov/pubmed/9871940

Tzschentke, T. M. (2007). Measuring reward with the conditioned place preference (CPP) paradigm: update of the last decade. Addiction Biology, 12(3-4), 227-462. doi:10.1111/j.1369-1600.2007.00070.x 
United Nations Office on Drugs and Crime (2012). World Drug Report. Retrieved from http://

www.unodc.org/documents/data-and-analysis/WDR2012/WDR_2012_web_small. pdf

Walker, C., \& Streisinger, G. (1983). Induction of Mutations by gamma-Rays in Pregonial Germ Cells of Zebrafish Embryos. Genetics, 103(1), 125-36. Retrieved from

http://www.pubmedcentral.nih.gov/articlerender.fcgi?artid=1202017\&tool=pmcentr ez\&rendertype $=\mathrm{abstract}$

Wang, Y., Takai, R., Yoshioka, H., \& Shirabe, K. (2006). Characterization and expression of serotonin transporter genes in zebrafish. The Tohoku Journal of Experimental Medicine, 208(3), 267-74. Retrieved from http://www.ncbi.nlm.nih.gov/pubmed/16498236

Winston, A. P. (2005). Neuropsychiatric effects of caffeine. Advances in Psychiatric Treatment, 11(6), 432-439. doi:10.1192/apt.11.6.432

Wong, K., Elegante, M., Bartels, B., Elkhayat, S., Tien, D., Roy, S., ... Kalueff, A. V. (2010). Analyzing habituation responses to novelty in zebrafish (Danio rerio). Behavioural Brain Research, 208(2), 450-7. doi:10.1016/j.bbr.2009.12.023

Wong, K., Stewart, A., Gilder, T., Wu, N., Frank, K., Gaikwad, S., ... Kalueff, A. V. (2010). Modeling seizure-related behavioral and endocrine phenotypes in adult zebrafish. Brain Research, 1348, 209-15. doi:10.1016/j.brainres.2010.06.012

World Health Organization (2011). Global status report on alcohol and health. Retrieved from 
http://www.who.int/substance_abuse/publications/global_alcohol_report/msbgsrupr ofiles.pdf

Yates, J. R., Marusich, J. a, Gipson, C. D., Beckmann, J. S., \& Bardo, M. T. (2012). High impulsivity in rats predicts amphetamine conditioned place preference. Pharmacology, Biochemistry, and Behavior, 100(3), 370-6.

doi:10.1016/j.pbb.2011.07.012

Zakharova, E., Leoni, G., Kichko, I., \& Izenwasser, S. (2009). Differential effects of methamphetamine and cocaine on conditioned place preference and locomotor activity in adult and adolescent male rats. Behavioural Brain Research, 198(1), 4550. doi:10.1016/j.bbr.2008.10.019

Zhdanova, I. V. (2011). Sleep and its regulation in zebrafish. Reviews in the Neurosciences, 22(1), 27-36. doi:10.1515/RNS.2011.005

Ziv, L., Muto, A., Schoonheim, P. J., Meijsing, S. H., Strasser, D., Ingraham, H. A., ... Baier, H. (2013). An affective disorder in zebrafish with mutation of the glucocorticoid receptor. Molecular Psychiatry, 18(6), 681-91. doi:10.1038/mp.2012.64 